\title{
Synthèse des tolérances thermiques des principales espèces de poissons des rivières et fleuves de plaine de l'ouest européen
}

\section{Synthesis on thermal tolerances of the principal freshwater fish species of large Western Europe rivers}

\author{
L. $\operatorname{Tissot}^{(1)}$, Y. Souchon ${ }^{(2)}$ \\ (1) EDF Recherche et Développement, Laboratoire National d'Hydraulique et Environnement, 6 quai Watier, \\ 78401 Chatou Cedex, France \\ (2) Laboratoire d'Hydroécologie Quantitative, MAEP, Pôle Études \& Recherche «Hydroécologie des cours \\ d'eau » ONEMA/CEMAGREF, 3bis quai Chauveau, CP 220, 69336 Lyon Cedex 09, France
}

\begin{abstract}
Résumé - L'effet de l'élévation de la température des cours d'eau sur le milieu aquatique a été largement débattu à la fin des années 70 qui ont vu le développement des centrales thermiques de grande puissance. Ces études ont généré de nombreux programmes de recherche sur le sujet. Le réchauffement climatique actuel et en particulier l'épisode caniculaire de 2003 a mis de nouveau ce thème sur le devant de la scène. Le travail de synthèse présenté a consisté à analyser des données historiques et contemporaines présentes dans la littérature, afin de mettre à jour les connaissances de base sur les tolérances thermiques de 19 espèces de poissons d'eau douce. Cette synthèse a ensuite été validée et complétée par des spécialistes européens. Elle s'est appuyée sur plus de 300 références qui détaillent les tolérances thermiques de chacun des stades du cycle de vie des espèces étudiées: la reproduction, les stades embryonnaire, larvaire, juvénile et adulte. Cette mise à jour est essentielle pour comprendre les tendances d'évolution déjà constatées des communautés de poissons et prévoir les futures évolutions selon les scénarios de changement climatique.
\end{abstract}

Mots clés - poissons, température, tolérance thermique, réchauffement

\begin{abstract}
The industrial cooling water effects on fish communities were widely debated at the end of the seventies, as large thermal power plants were being developed. This has generated numerous research programs on thermal fish tolerances. The recent warming period and especially the 2003 heat-wave event have placed again the thermal biology at the front of the scene. The work presented has consisted in analyzing historical and contemporary literature to update the basic knowledge on thermal tolerances of 19 riverine fish species. These data were validated and completed by European fish specialists. We finally proposed a synthesis, based on more than 300 references, which detailed thermal tolerances for the entire cycle life: reproduction, embryonic, larval, juvenile and adult life stages. We think that the updated material is of great importance to understand observed trends in fish communities or to forecast future behaviors in scenarios of climate changes.
\end{abstract}

Key words - fishes, temperature, thermal tolerance, warming 


\section{INTRODUCTION}

L'effet de la température de l'eau sur les organismes aquatiques a constitué un thème central des recherches en écologie aquatique dans les années 70 , en réponse aux interrogations de la société sur l'impact des installations industrielles nécessitant de l'eau comme source froide dans leurs procédés, eau qui est ensuite restituée plus chaude dans les cours d'eau (Leynaud, 1967; Talmage, 1977). En lien avec la mise en place de tours de refroidissement atmosphérique, ce thème n'a plus occupé le devant de la scène pendant près de 3 décades, puis il a été à nouveau extrêmement questionné d'abord à large échelle, à l'occasion de l'épisode caniculaire européen de 2003 (De Bono et al., 2004) et surtout par rapport aux enregistrements de réchauffement climatique (au $20^{\mathrm{e}}$ siècle augmentation de la température moyenne de l'air par décade $+0,10{ }^{\circ} \mathrm{C}$ à $0,16{ }^{\circ} \mathrm{C}$, Intergovernmental Panel on Climate Change, 2007) et eu égard aux prévisions des modèles du GIEC (prévision d'augmentation en 2090-2099 par rapport à 1980-1999 : $+1,8^{\circ} \mathrm{C}$ à $4,0{ }^{\circ} \mathrm{C}$ selon les scénarios, GIEC, 2007). À une échelle plus locale, des perturbations plus insidieuses du régime thermique sont également documentées depuis quelques années: effets de l'urbanisation et réchauffement de l'eau par les stations d'épuration de l'eau (Kinouchi, $2007:+0,11^{\circ} \mathrm{C}$ à $0,22{ }^{\circ} \mathrm{C}$ par an aux sites affectés par les rejets d'une grande agglomération comme Tokyo) ou par l'augmentation des surfaces imperméabilisées, effets des retenues pouvant se traduire par l'augmentation ou la diminution de la température en aval
(Olden \& Naiman, 2010; Quinn \& Adams, 1996 in Petersen \& Kitchell, 2001 : seuil de $15,5{ }^{\circ} \mathrm{C}$ plus précoce de 1 mois au printemps et plus tardif de 15 jours à l'automne, Columbia River, Bonneville Dam) ou encore effets du déficit de cordons boisés riverains laissant plus de surfaces en eau livrées aux radiations solaires directes (Bartholow, 2000 en moyenne sur plusieurs cas, $+3{ }^{\circ} \mathrm{C}$ à $6{ }^{\circ} \mathrm{C}$ pour la température moyenne journalière et $+3^{\circ} \mathrm{C}$ à $8{ }^{\circ} \mathrm{C}$ pour la température maximum; Burton \& Likens, 1973 in Kaushal et al., 2010 : maximum journalier de $+4{ }^{\circ} \mathrm{C}$ à $5{ }^{\circ} \mathrm{C}$ suite à une coupe à blanc; Moore et al., 2005).

En écologie, la température est une variable clé, plus délicate à interpréter qu'en physique, dans la mesure où il faut tenir compte non seulement des valeurs instantanées, mais aussi de leur répartition annuelle, saisonnière et journalière. Ainsi, les cumuls de températures dans une gamme favorable pour les organismes régissent leur physiologie (somme de degrés-jours ou somme des températures moyennes journalières au dessus de $0{ }^{\circ} \mathrm{C}$ ou d'un seuil connu pour une espèce cible, Allan, 1995). D'un point de vue fonctionnel, la température agit sur la physico-chimie (ex. solubilité de l'oxygène), sur la vitesse de recyclage des nutriments ou de décomposition de la matière organique et sur le fonctionnement métabolique du vivant (ex. production primaire). Des périodes critiques pour les organismes aquatiques peuvent survenir par exemple en cas de simultanéité entre un stress hydrique de type étiage bas et une température élevée dans les milieux aquatiques (Miller et al., 2007). À l'échelle des 
biotes, en particulier des poissons poïkilothermes, la température du milieu environnant influence le métabolisme (Brown et al., 2004; Gillooly et al., 2001), le taux d'ingestion et de digestion (Brett \& Higgs, 1970; Tseitlin, 1980), les cycles de reproduction (De Vlaming, 1972), avec des exigences qui varient au cours de l'ontogénèse (Lapkin et al., 1983 in Raat, 1988). Les comportements de nage (Mann \& Bass, 1997; Ojanguren \& Brana, 2000), les vitesses de croissance (Wieser et al., 1988; Wolter, 2007) sont également sous la dépendance de la température. Par conséquent la dynamique des populations et des communautés, leur composition relative (Kishi et al., 2005) et leur structure en taille (Daufresne et al., 2009) traduisent les réponses biotiques aux régimes thermiques ambiants. En fonction de leurs exigences et de leurs tolérances thermiques, les espèces ectothermes vont ou non se satisfaire de nouvelles conditions environnementales : certaines vont décliner, voire disparaître de zones géographiques (comme certains mollusques de la Saône, Mouthon \& Daufresne, 2006; Webb, 1996), d'autres au contraire vont étendre leur aire de répartition (Hickling et al., 2006). Pour bien comprendre les changements observés ou bien pour anticiper le statut futur des espèces, il est essentiel d'en connaître les preferendums thermiques; l'interprétation des températures devra être toutefois nuancée dans la mesure où certains comportements (Davey et al., 2006; aptitude à la dispersion, faculté à trouver des zones thermiques refuges, Torgersen et al., 1999) ou certaines aptitudes phénotypiques (plasticité lors du développement, Schaefer \& Ryan, 2006) permettent à certaines espèces de survivre à des épisodes thermiques critiques.

Des synthèses récentes sur les exigences thermiques des espèces de poissons se sont focalisées sur les températures lors des phases embryonnaire et de reproduction (Teletchea et al., 2007, 2009), pour des espèces pouvant présenter un intérêt zootechnique. Des synthèses partielles plus anciennes ont également été publiées (Alabaster \& Lloyd, 1982; Hokanson, 1977; Küttel et al., 2002; Mann, 1996; Teletchea et al., 2008), mais il n'existait pas à notre connaissance de synthèse récente accessible pour tous les stades vitaux des espèces de poissons communément rencontrées dans les cours d'eau moyens et grands.

Cet article répond à ce besoin de synthèse des connaissances sur les profils thermiques de la vingtaine d'espèces qui constitue le noyau central des communautés de poissons des cours d'eau de rang fluvial égal ou supérieur à 5 de l'ouest européen, hors salmonidés.

Quand elles étaient disponibles, nous avons exploité les exigences pour tous les stades de développement (phase adulte, reproduction, vie embryonnaire, vie larvaire, phase juvénile), aussi bien pour les observations dans le milieu naturel qu'en milieu contrôlé. La littérature accessible par les bases de données documentaires numérisées occulte des textes intéressants, souvent anciens, parfois de littérature grise ou des textes en langue native (allemand, français) mal référencés. Nous avons fait un effort particulier pour exploiter ceux dont nous 
Tableau I. Nom vernaculaire, nom latin et code des 19 espèces étudiées.

Table I. French name, Latin name and code of the 19 studied species.

\begin{tabular}{|l|l|l|l|}
\hline Code & \multicolumn{1}{|c|}{ Famille } & Nom vernaculaire & \multicolumn{1}{|c|}{ Nom latin } \\
\hline ABL & Cyprinidae & ablette & Alburnus alburnus \\
BAF & Cyprinidae & barbeau fluviatile & Barbus barbus \\
BLN & Cyprinidae & blageon & Telestes souffia \\
BOU & Cyprinidae & bouvière & Rhodeus sericeus \\
BRB & Cyprinidae & brème bordelière & Blicca bjoerkna \\
BRE & Cyprinidae & brème commune & Abramis brama \\
BRO & Esocidae & brochet & Esox lucius \\
CHE & Cyprinidae & chevesne & Leuciscus cephalus \\
GAR & Cyprinidae & gardon & Rutilus rutilus \\
GOU & Cyprinidae & goujon & Gobio gobio \\
GRE & Percidae & grémille & Gymnocephalus cernuus \\
HOT & Cyprinidae & hotu & Chondrostoma nasus \\
PER & Percidae & perche fluviatile & Perca fluviatilis \\
PES & Centrarchidae & perche soleil & Lepomis gibbosus \\
SAN & Percidae & sandre & Stizostedion lucioperca \\
SIL & Siluridae & silure & Silurus glanis \\
SPI & Cyprinidae & spirlin & Alburnoides bipunctatus \\
TOX & Cyprinidae & toxostome & Chondrostoma toxostoma \\
VAN & Cyprinidae & vandoise & Leuciscus leuciscus \\
\hline
\end{tabular}

avons pu disposer; nous avons complété ce travail par une enquête directe auprès d'auteurs européens (23 interrogés) susceptibles de détenir ce type d'informations. Pour les 19 espèces étudiées (Tab. I), la compilation a porté sur plus de 300 références. Toute l'information bibliographique détaillée est mise à disposition en annexe 1 .

Compte-tenu de la disparité intrinsèque des sources et des modes de calcul des grandeurs thermiques d'intérêt (Beitinger \& Bennett, 2000 listent 450 tolérances pour 116 espèces), nous faisons un point préalable sur les définitions retenues. Les données de chaque espèce analysée sont ensuite commentées. Enfin, nous proposons une lecture synthétique des plages thermiques tolérées en regroupant les espèces aux exigences de même nature.

\section{DÉFINITIONS ET MÉTHODOLOGIE}

\subsection{Gamme des températures optimales}

Dans la gamme des températures optimales, le poisson se nourrit et ne présente aucun signe de comportement anormal, il n'est pas en état de stress (Elliott, 1981). Dans cette gamme de température, des régulations physiologiques interviennent efficacement pour permettre au poisson 
de supporter des températures relativement élevées : induction des protéines de stress thermique, modification des acides gras membranaires (Iwama et al., 1998) La borne inférieure de cette gamme est la température optimale minimale et la borne supérieure est la température optimale maximale.

\subsection{Effet sur la durée du développement}

Chez les poissons, la gamétogenèse et la ponte s'effectuent dans une gamme de températures définies. Comme pour les autres fonctions physiologiques, il existe une gamme de températures optimales pour la reproduction et des seuils thermiques inférieurs et supérieurs en deçà et audessus desquels la gamétogenèse et la fraie sont inhibées.

Le nombre de degrés jours est l'unité permettant de déterminer la durée de développement embryonnaire en fonction de la température moyenne journalière : par exemple, durée entre deux stades de développement définis. Le nombre de degrés jours s'obtient en additionnant la température moyenne de chaque jour entre les deux stades de développement considérés. La plupart du temps, ce nombre est mesuré en laboratoire à une température donnée, inchangée au cours de l'expérience, et se calcule alors en multipliant cette température avec la durée de développement embryonnaire étudiée (en nombre de jours). En toute rigueur, il faut vérifier que cette relation est constante sur une plage de températures pour être affichée en tant que durée en degrés jours.

\subsection{Température critique et température létale établies par l'expérimentation}

\subsubsection{Définitions}

Le stress thermique induit trois phases successives de réactions chez le poisson. Elles ont été identifiées en laboratoire par Elliott (1981). La première phase est définie par une réticence à s'alimenter, de brusques séquences d'activité pendant lesquelles le poisson se heurte à la paroi de l'aquarium, des instants de perte de l'équilibre, une défécation et de rapides mouvements respiratoires. Dans la seconde phase, le poisson devient immobile avec de brusques mais faibles mouvements de nage, il flotte la plupart du temps sur le côté ou sur le dos, il peut changer rapidement de couleur et augmente la fréquence de ses mouvements de ventilation. La dernière phase est caractérisée par des mouvements restreints uniquement visibles au niveau de l'opercule, des nageoires pectorales et des yeux, jusqu'à la mort du poisson. Si les poissons sont introduits dans de l'eau plus fraîche et mieux oxygénée, ceux qui ont atteint la dernière phase de stress ne récupèrent pas et meurent (expériences d'Elliott, 1981 sur la truite et de Cocking, 1959 sur le gardon).

\section{Gamme de résistance à la température}

Lors des épisodes de stress thermique, les mécanismes physiologiques de protection sont débordés par la rapidité d'apparition des effets, le poisson peut toutefois « résister" un certain temps sans subir de graves dommages ou avec un taux de survie 
élevé. Cette gamme de températures dans laquelle le poisson subit le stress thermique est appelée "zone de résistance ".

\section{Gamme de tolérance à la température}

À l'intérieur de la zone de résistance existe une «zone de tolérance » dans laquelle le comportement de stress ne se manifeste pas et où la survie du poisson est théoriquement assurée. Dans cette zone, le poisson survit au choc thermique mais ses fonctions physiologiques (alimentation, respiration, croissance) peuvent être affectées. La tolérance thermique n'a de ce fait de sens qu'à court terme (un jour à quelques jours au maximum). Cette zone est le plus souvent délimitée par les températures létales déterminées dans la gamme de 24 heures à 48 heures. Les données collectées sur de plus longues durées (7 jours) commencent quant à elles à intégrer des effets physiologiques autres que le stress thermique.

\section{Température létale : mort instantanée}

Dans la zone de résistance, la température à laquelle tous les poissons meurent en moins de dix minutes correspond à la température de mort instantanée (Cocking, 1959).

\subsubsection{Modalités expérimentales de déterminations de ces températures}

Deux méthodes expérimentales ont été développées pour caractériser le stress thermique principalement au cours des années $60-70$ : la méthode de la température critique (Critical Thermal Method - CTM) et la technique de la température provoquant les premières mortalités (Incipient Lethal Temperature - ILT).

Ces deux méthodes de détermination de la tolérance thermique des espèces piscicoles n'aboutissent pas aux mêmes températures limites du fait de conditions expérimentales différentes, bien qu'elles soient dans les deux cas corrélées positivement avec la température d'acclimatation.

\section{Température d'acclimatation}

La température d'acclimatation est la température à laquelle sont exposés expérimentalement des lots de poissons plusieurs jours durant (au moins une semaine selon Fry, 1971) avant un test de tolérance thermique (Armour, 1991). La température d'acclimatation se situe dans la zone de tolérance du poisson.

\section{Détermination des températures critiques}

La CTM est une technique de détermination des tolérances thermiques des poissons en laboratoire. Elle a été initialement mise au point pour l'étude des amphibiens puis élargie aux poissons (Cowles \& Bogert, 1944).

Un échantillon de poissons acclimatés à une température donnée est soumis à un accroissement (ou à une diminution) linéaire de la température jusqu'à un seuil sublétal (mais proche de la température létale) prédéfini. $\mathrm{Ce}$ seuil qui correspond aux valeurs CT maximum ou CT minimum est le point auquel les mouvements de locomotion du poisson deviennent désordonnés et 
ne lui permettent plus de fuir des conditions qui peuvent finalement le mener à la mort (Houston, 1982). Dans cette approche, la grandeur thermique de la température létale est estimée sans tuer d'individus, les dommages observés sont réversibles, il n'y a donc pas d'effet létal. Le choix du critère sublétal peut varier selon les auteurs : Beitinger et al. (2000) citent par exemple le déclenchement de spasmes musculaires violents (Matthews \& Maness, 1979), la perte de l'équilibre (Watenpaugh et al., 1985) ou l'ouverture des opercules (Middaugh et al., 1975).

Les températures limites obtenues avec ce type d'expérimentation sont souvent plus élevées que celles issues de la méthode de I'ILT en relation avec la durée d'exposition aux températures testées généralement plus courte (Todd et al., 2008).

\section{Détermination des températures létales}

L'ILT est la méthode d'approche de la température létale en laboratoire la plus ancienne et la plus répandue. Elle a été développée par Fry et al. (1942), à partir des recherches sur la relation dose thermique-réponse (Bliss, 1937).

Des lots de poissons acclimatés à une température donnée sont transférés brutalement dans une eau plus chaude ou plus froide que la température d'acclimatation pendant une durée variable. La proportion de poissons morts dans chaque lot est alors mesurée. Un traitement statistique de ces données permet de déterminer la température létale.

La température létale (TLx,y) est la température causant la mort d'une fraction d'un lot de poissons $(x)$ pour une durée d'exposition donnée (y). Les temps mentionnés vont en général de 100 minutes à 24 heures, voire parfois 96 heures. Par exemple TL50,24 est la température létale pour $50 \%$ du lot testé après 24 heures. Lorsque le taux de mortalité n'est pas mentionné, il s'agit de la TL50, qui est la grandeur déterminée avec la plus faible incertitude dans les expérimentations. Par analogie, la CL50 pour le dioxygène correspond à la concentration en dioxygène létale pour la moitié des individus testés à une température d'acclimatation donnée.

Des essais pratiqués dans de l'eau plus chaude ou plus froide que la température d'acclimatation fournissent respectivement des températures létales supérieures et des températures létales inférieures.

Pour un stade biologique donné (œufs, larves, juvéniles, adultes), la température létale dépend de la température d'acclimatation et de la durée d'exposition à l'échauffement ou au refroidissement; elle croît lorsque la température d'acclimatation augmente, puis atteint une valeur seuil. La littérature en langue anglaise désigne généralement cette valeur seuil par «Ultimate Incipient Lethal Temperature » (UILT) tandis que les températures létales situées dans la phase de dépendance à la température d'acclimatation sont appelées « Incipient Lethal Temperature ». Rappelons que ces grandeurs thermiques sont des TL50.

\subsubsection{Signification des températures critique et létale}

D'après leur mode expérimental de détermination, ces grandeurs représentent un stress thermique létal 
(température létale) ou sublétal (température critique). Dans un cas le choc thermique cause des dommages irréversibles conduisant à la mort, dans l'autre l'échauffement entraîne des mouvements désordonnés qui cessent lorsque le poisson est remis dans une eau à la température d'acclimatation. II ne s'agit donc pas dans les deux cas d'effets physiologiques se manifestant à long terme du fait de l'accroissement progressif d'une dette d'oxygène ou de la dégradation du bilan énergétique global, mais de réactions rapides à la variation de température induisant à court terme un désordre physiologique.

Le schéma présenté sur la figure 1a fait apparaître trois régions :

- la zone de tolérance thermique, compatible avec la survie des poissons;

- la zone de résistance thermique;

- la zone de mortalité instantanée.

\subsection{Méthodologie adoptée par les auteurs}

Les auteurs ont pris le parti de synthétiser l'ensemble des données recueillies sur les tolérances thermiques des espèces en proposant deux gammes de température caractéristiques de chaque stade de vie lorsque la donnée est suffisante (Fig. 1b) :

- une gamme de températures optimales qui correspond à la gamme où la majorité des données mentionnées sous ce nom dans la littérature se situent;

- une gamme optimale élargie, correspondant aux températures extrêmes minimales et maximales supportées, dont les limites correspondent à celles de la gamme de résistance définie ci-dessus. Cette gamme est limitée par les CT max établies en laboratoire lorsqu'elles existent. Pour le stade embryon, il s'agit souvent de la température létale (les températures limites entre l'optimum et la température létale sont difficiles à mesurer).

De manière générale, les données d'observations sont préférées aux ouvrages de compilation. Une attention particulière a également été portée à la cohérence des données pour établir les gammes d'un stade de vie donné et entre stades différents d'une même espèce. Lorsque les données étaient suffisamment robustes sur les autres stades de vie et insuffisantes pour le juvénile ou l'adulte de la même espèce, certaines limites de gamme ont été transposées d'un stade à l'autre selon les règles :

- gamme du juvénile = gamme de l'adulte;

- gamme optimum du juvénile = gamme élargie de la larve.

\subsection{Les paramètres liés à la température}

Les espèces piscicoles ont des préférences d'habitat sensiblement différentes selon leur stade de vie. Les préférences thermiques se traduisant également différemment selon les stades, les paramètres retenus varient pour chacun d'eux. Chaque période du cycle de vie du poisson est donc examinée séparément. 


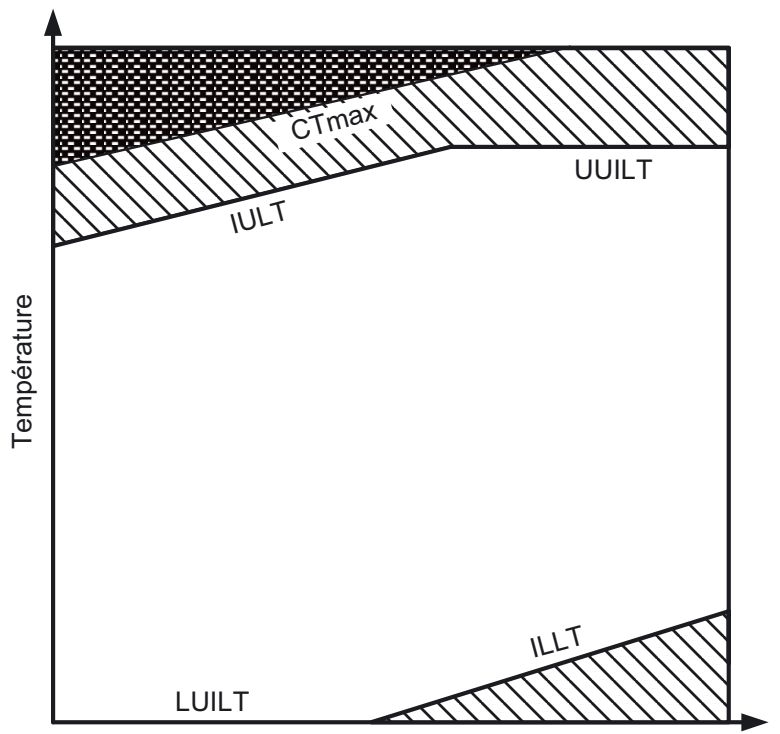

Température d'acclimatation

a

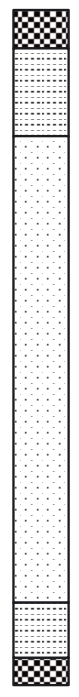

b

IULT : Incipient Upper Lethal Temperature UUILT : Upper Ultimate Incipient Lethal Temperature ILLT : Incipient Lower Lethal Temperature LUILT : Lower Ultimate Incipient Lethal Temperature CTmax : Seuil maximum établi par CTM / Maximum threshold established by CTM

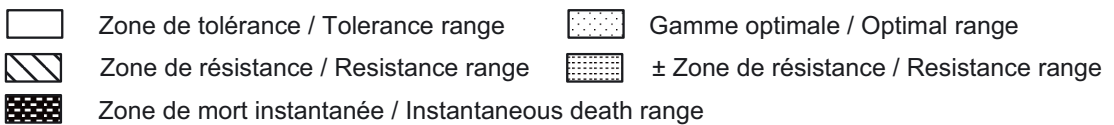

Fig. 1. Représentation schématique de différentes grandeurs thermiques (d'après Beitinger et al., 2000; Todd et al., 2008) (a) et des gammes de tolérance retenues par les auteurs (b).

Fig. 1. Diagram of the different thermal quantities (Beitinger et al., 2000; Todd et al., 2008) (a) and of the tolerance ranges identified by the authors (b).

Pour les stades adulte et juvénile, les températures optimales de vie sont répertoriées. Les températures limites obtenues en laboratoire (expériences de type CTM ou ILT) sont également des données principalement connues pour ces stades. Enfin, lorsque des températures de mortalité d'individus sont constatées in situ, elles sont mentionnées.
Pour l'embryon et la larve, il est rajouté la durée en nombre de degrés jours de chacun de ces deux stades, avec la température de l'eau associée lorsqu'elle est connue.

Les températures seuils de début et de fin de reproduction sont également des paramètres retenus.

Pour chaque donnée de température rencontrée, certaines précisions 
sur l'étude qui la mentionne sont systématiquement conservées lorsqu'elles sont précisées (localisation de l'étude, température relevée in situ ou issue d'expériences en laboratoire, citations d'autres études). Ces renseignements permettront ensuite de comparer des valeurs de même nature entre elles.

Parmi les différents paramètres rencontrés, certains sont préférentiellement recherchés compte tenu de leur signification écologique.

$\mathrm{Au}$ sein de la plage des températures optimales, le poisson ne subit pas de stress et vit sans contrainte. Cette donnée est donc essentielle à une bonne synthèse des tolérances thermiques.

Parmi les différentes données expérimentales, I'IULT à 24 heures est préférée aux données obtenues avec des durées d'exposition plus courtes. En effet, les températures limites obtenues avec des temps d'exposition plus courts (par exemple 100 ou 1000 minutes) provoquent des séquelles : la dernière phase du stress thermique décrit par Elliott (1981) est alors dépassée.

\subsection{Les espèces de poissons}

La synthèse porte sur 19 espèces de poissons, les plus représentées dans les portions de cours d'eau de taille moyenne à grande (rang fluvial égal ou supérieur à 5) de l'ouest de l'Europe. Une recherche bibliographique approfondie de leurs tolérances thermiques a été conduite. Ces 19 espèces non migratrices sont : le blageon $^{(1)}$, le toxostome ${ }^{(1)}$, le hotu,

\footnotetext{
(1) Espèce Natura 2000.
}

le goujon, le chevesne, la vandoise, le spirlin, le barbeau fluviatile, la bouvière(1), le gardon, l'ablette, la brème commune, la brème bordelière, la perche fluviatile, la grémille, le sandre, le brochet, la perche soleil et le silure (Tab. I).

\subsection{Récapitulatif des données recueillies}

Parmi les 390 références consultées et répertoriées mentionnant des données de tolérances thermiques sur les espèces de poissons étudiées, près de 300 sont effectivement citées dans ce rapport. Un quart environ de ces références sont des compilations de données (des ouvrages de portée générale sur les poissons d'eau douce ou des synthèses bibliographiques sur l'étude du facteur température), les trois quarts étant des observations. Seuls $35 \%$ des températures trouvées sont issues d'expériences en laboratoire $(65 \%$ sont issues d'études in situ).

Le tableau II synthétise le nombre de données recueillies (tous types confondus), pour les différents stades de chacune des 19 espèces ciblées dans cette étude. Une gamme de température optimale est considérée comme une seule donnée lorsqu'elle est proposée dans une même référence.

De manière générale, les tolérances thermiques des différents stades du cycle de vie du poisson ne sont pas étudiées de façon homogène : le stade larvaire et le stade juvénile sont très peu documentés en comparaison des 
Tableau II. Récapitulatif du nombre de données recueillies pour chaque stade de vie des espèces (classées selon la typologie de Verneaux, 1981).

Table II. Summary of the number of data for each species life stage (sorted according to Verneaux typology, 1981).

\begin{tabular}{|c|c|c|c|c|c|c|}
\hline Espèces & Embryon & Larve & Juvénile & Adulte & $\begin{array}{c}\text { Reproduction } \\
\text { (+gamétogenèse) }\end{array}$ & TOTAL \\
\hline Blageon & 1 & 0 & 2 & 2 & 3 & 8 \\
Toxostome & 1 & 1 & 0 & 2 & 5 & 9 \\
Hotu & 12 & 6 & 1 & 2 & 14 & 35 \\
Goujon & 3 & 1 & 1 & 11 & 7 & 23 \\
Chevesne & 12 & 8 & 1 & 5 & 5 & 31 \\
Vandoise & 21 & 4 & 1 & 1 & 17 & 44 \\
Spirlin & 3 & 2 & 0 & 2 & 7 & 14 \\
Barbeau fluviatile & 15 & 3 & 4 & 3 & 9 & 34 \\
Bouvière & 0 & 0 & 0 & 3 & $4(+1)$ & $7(+1)$ \\
Gardon & 5 & 2 & 10 & 12 & 15 & 44 \\
Ablette & 1 & 1 & 0 & 4 & 7 & 13 \\
Brème commune & 8 & 2 & 1 & 9 & 13 & 33 \\
Brème bordelière & 2 & 0 & 0 & 2 & 5 & 9 \\
\hline Perche fluviatile & 11 & 8 & 6 & 18 & 16 & 59 \\
Grémille & 6 & 2 & 3 & 2 & $8(+1)$ & $21(+1)$ \\
Sandre & 10 & 4 & 2 & 5 & 13 & 34 \\
Brochet & 10 & 3 & 3 & 11 & 14 & 41 \\
Perche soleil & 1 & 2 & 3 & 18 & 4 & 28 \\
Silure & 4 & 2 & 0 & 9 & 8 & 23 \\
\hline TOTAL & 126 & 51 & 38 & 121 & 162 & 498 \\
\hline
\end{tabular}

Espèce bénéficiant de moins de 10 données/Species benefiting from less than 10 data

autres. Le stade embryonnaire est renseigné de manière très peu homogène selon les espèces, tandis que les adultes et la reproduction sont les phases les plus étudiées du cycle de vie en regard de la température. Malgré les efforts de recherche réalisés, quatre espèces, caractérisées chacune par moins de dix données, sont particulièrement peu connues en termes de tolérance thermique : il s'agit du blageon, du toxostome, de la bouvière et de la brème bordelière (Tab. II, en grisé).

\section{RÉSULTATS}

Les données de tolérances thermiques recueillies par stade au sein de chaque espèce sont commentées ci dessous et présentées intégralement en annexe 1. Les espèces sont classées par famille selon 
leur zonation longitudinale théorique dans les cours d'eau (Verneaux, 1981). Des gammes de température optimale et des gammes plus larges dont les bornes extrêmes correspondent à celles de la zone de résistance sont proposées pour chaque stade lorsque la donnée existe ou peut être raisonnablement estimée (voir méthodologie adoptée par les auteurs paragraphe 2.4).

\subsection{Famille des Cyprinidae}

\subsubsection{Le blageon (Telestes souffia)}

La durée du stade embryonnaire du blageon est estimée à 7 jours à 13,9 ${ }^{\circ} \mathrm{C}$ par Bohl et al. (2004). Ces mêmes auteurs (Bohl et al., 2004) maintiennent en aquarium des embryons de blageon entre $13,9{ }^{\circ} \mathrm{C}$ et $18,5^{\circ} \mathrm{C}$.

L'optimum de température pour le stade juvénile semble se situer entre $13^{\circ} \mathrm{C}$ et $15^{\circ} \mathrm{C}$ avec une température extrême minimale de $10^{\circ} \mathrm{C}$ (Bohl et al., 2004).

Pour le stade adulte, l'optimum se situe entre $10^{\circ} \mathrm{C}$ et $18^{\circ} \mathrm{C}$ (Ginot et al., 1996; Fishbase), avec des températures extrêmes de $1,9{ }^{\circ} \mathrm{C}$ à $4{ }^{\circ} \mathrm{C}$ pour le minimum (Bohl et al., 2004) et de $27^{\circ} \mathrm{C}$ pour le maximum (Ginot et al., 1996 ; Stankovitch, 1921).

La reproduction enfin a lieu entre $12{ }^{\circ} \mathrm{C}$ (fiche Natura 2000) et $15^{\circ} \mathrm{C}$ (Bohl et al., 2004), avec une température extrême maximale estimée à $19{ }^{\circ} \mathrm{C}$ (Bohl et al., 2004). Spillman (1962 in Schwarz, 1996) mentionne un optimum restreint entre $11{ }^{\circ} \mathrm{C}$ et $13^{\circ} \mathrm{C}$.

\subsubsection{Le toxostome (Chondrostoma toxostoma)}

Spillman (1961) estime que l'embryon de toxostome éclot entre 12 et 15 jours après la ponte (mais sans préciser de température). Gozlan et al. (1999) mentionnent un élevage en aquarium à $16{ }^{\circ} \mathrm{C}$ et une durée d'incubation de 8 jours.

Ces derniers auteurs (Gozlan et al., 1999) ont observé la croissance larvaire en milieu naturel à des températures comprises entre $14^{\circ} \mathrm{C}$ et $18^{\circ} \mathrm{C}$.

La gamme des température optimales pour le stade adulte s'établit entre $16^{\circ} \mathrm{C}$ et $25^{\circ} \mathrm{C}$ (Gozlan, 1998).

Bruslé \& Quignard (2001) évaluent l'optimum de reproduction entre $9^{\circ} \mathrm{C}$ et $1{ }^{\circ} \mathrm{C}$ tandis que Perche (1948 dans le bassin du Doubs, in Spillmann, 1961) et Lelek (1987) mentionnent un début de reproduction à $13{ }^{\circ} \mathrm{C}$. La fiche Natura 2000 de l'espèce rapporte quant à elle une reproduction à des températures intermédiaires entre ces deux références, entre $11^{\circ} \mathrm{C}$ et $13^{\circ} \mathrm{C}$ sur le cours inférieur du Verdon. II semble admis par les ichtyologistes que les conditions de température du toxostome sont proches de celles du hotu, ce qui les situeraient entre $8^{\circ} \mathrm{C}$ et $14{ }^{\circ} \mathrm{C}$. Toutefois, Chappaz et al. (1989 in Maier et al., 1995) ont observé la fraie dans le Verdon un mois après celle du hotu, soit entre fin mai et début juin. Les températures optimales pour la reproduction du toxostome semblent donc se situer entre $9^{\circ} \mathrm{C}$ et $14{ }^{\circ} \mathrm{C}$.

\subsubsection{Le hotu (Chondrostoma nasus)}

Les températures optimales au développement de l'embryon de hotu 
sont comprises entre $10{ }^{\circ} \mathrm{C}$ (Penaz, 1974b en République Tchèque) et $17{ }^{\circ} \mathrm{C}$ (Müller, 1997 in Küttel et al., 2002), quatre références mentionnant des optimums dans cette plage (Herzig \& Winkler, 1985 in Küttel et al., 2002; Keckeis et al., 2001 en Autriche, in Schiemer et al., 2003; Lusk, 1995 en République Tchèque; Penaz, 1974b). Les températures extrêmes tolérées sont pour leur part estimées entre $8^{\circ} \mathrm{C}$ (Kamler et al., 1998; Prokes \& Penaz, 1978) et $19{ }^{\circ} \mathrm{C}$ (Schiemer et al., 2004) avec une température létale mentionnée à $20^{\circ} \mathrm{C}$ par Kamler et al. (1998 in Kamler, 2002).

Herzig \& Winkler (1985) ont établi une équation reliant la durée d'incubation (D en jours, $50 \%$ d'éclosion) à la température $\left(\mathrm{T}\right.$ en $\left.{ }^{\circ} \mathrm{C}\right)$ :

$$
\mathrm{D}=1258 /(T-2,62)^{1,994}
$$

soit, 23,1 jours à $10^{\circ} \mathrm{C}$ et 5,8 jours à $17^{\circ} \mathrm{C}$, plage de validité de l'équation.

Pour la larve, la température optimale est comprise entre $15^{\circ} \mathrm{C}$ (Kamler et al., 1998; Penaz, 1974a in Schiemer et al., 2003 sur le Danube) et $25^{\circ} \mathrm{C}$ (Keckeis et al., 2001; Schiemer et al., 2004). Le maximum supporté est de l'ordre de $28{ }^{\circ} \mathrm{C}$ (Penaz, 1974a in Schiemer et al., 2003 sur le Danube; Schiemer et al., 2003; Spurny et al., 2004 in Wolnicki \& Gorni, 1994), donnée reprise dans deux articles (Schiemer et al., 2003; Wolnicki \& Gorni, 1994). La larve semble également tolérer des températures jusqu'à $10^{\circ} \mathrm{C}$ (Schiemer et al., 2004).

Au vu des résultats de pêches sur le Rhône, Ginot et al. (1996) établissent des températures optimales pour les juvéniles de hotu entre $7^{\circ} \mathrm{C}$ et $27^{\circ} \mathrm{C}$. II semble davantage réaliste de situer ces températures sur une gamme plus réduite, comparable à celle de l'embryon, entre $15{ }^{\circ} \mathrm{C}$ et $25^{\circ} \mathrm{C}$ (Kamler et al., 1998 ; Penaz, 1974b in Schiemer et al., 2003, 2004; Teletchea et al., 2008).

Bruslé \& Quignard (2001) sont les seuls à définir des seuils de tolérance thermique pour l'adulte : ils considèrent que la gamme de température pendant laquelle l'adulte s'alimente s'étend de $4{ }^{\circ} \mathrm{C}$ à $24^{\circ} \mathrm{C}$. Nous considérons néanmoins que l'optimum minimal semble raisonnablement se situer à des températures plus chaudes, semblables à celles du juvénile, autour de $15^{\circ} \mathrm{C}$.

La plupart des auteurs positionne la plage de température optimale pour la reproduction du hotu entre $8{ }^{\circ} \mathrm{C}$ et $14{ }^{\circ} \mathrm{C}$ (Banarescu, 1964; Bruslé \& Quignard, 2001; Keckeis et al., 2001; Penaz, 1974b in Schiemer et al., 2004; Penaz et al., 1995; Philippart, 1980; Poncin, 1996; Prokes \& Penaz, 1978; Teletchea et al., 2008). Les températures extrêmes permettant la reproduction sont de l'ordre de $6^{\circ} \mathrm{C}$ pour le minimum (Filipi, 1962 en Albanie; Herzig \& Winkler, 1985 in Küttel et al., 2002; Lusk, 1967) et de $16{ }^{\circ} \mathrm{C}$ pour le maximum (Alabaster \& Lloyd, 1982; Herzig \& Winkler, 1985 in Küttel et al., 2002).

\subsubsection{Le goujon (Gobio gobio)}

La durée du développement embryonnaire du goujon a été estimée en laboratoire entre 6,3 jours à $20{ }^{\circ} \mathrm{C}$ (Brunet \& Hoestlandt, 1972) et 6,9 jours à $18,5{ }^{\circ} \mathrm{C}$, soit environ 125 degrés jours, ce que confirment Teletchea et al. (2008). La gamme de température optimale est située 
entre $16{ }^{\circ} \mathrm{C}$ et $20{ }^{\circ} \mathrm{C}$ (Tocko, 1987 in Banarescu et al., 2003b).

La température optimale pour la larve est estimée à $20,5{ }^{\circ} \mathrm{C}$ par Telechea et al. (2008).

Ginot et al. (1996) observent des températures optimales pour le stade juvénile entre $7{ }^{\circ} \mathrm{C}$ et $27^{\circ} \mathrm{C}$ sur le Rhône.

Le stade adulte est le mieux renseigné pour le goujon : sa température optimale inférieure est établie à $7{ }^{\circ} \mathrm{C}$ (Ginot et al., 1996 sur le Rhône) et sa limite optimale supérieure à $30{ }^{\circ} \mathrm{C}$ (Elliott, 1981 in Küttel et al., 2002; Kryzhanovskii, 1949 in Alabaster \& Lloyd, 1982), avec trois références supplémentaires mentionnant une gamme plus restreinte (Banarescu et al., 2003a; Bernet, 1960 sur la Nivelle, in Hynes, 1970 ; Bruslé \& Quignard, 2001). Horoszewicz (1973 in Alabaster \& Lloyd, 1982) observe en Pologne une température létale in situ de $36,7{ }^{\circ} \mathrm{C}$ sur une retenue à $25,5^{\circ} \mathrm{C}$ (seuil sublétal atteint à $30,8-$ $30,9^{\circ} \mathrm{C}$ ). Cette même température létale est également mentionnée par Kryzhanovskii (1949 in Alabaster \& Lloyd, 1982). $36{ }^{\circ} \mathrm{C}$ est donc choisie par les auteurs comme température maximale supérieure supportée par le stade. La température extrême inférieure semble se situer autour de $5{ }^{\circ} \mathrm{C}$ (Banarescu, 1964). Quatre auteurs ont réalisé des tests en laboratoire pour identifier des températures limites. Les valeurs d'IULT mentionnées sont comprises entre $27,2^{\circ} \mathrm{C}$ (Alabaster, 1964) et $30{ }^{\circ} \mathrm{C}$ (Varley, 1967 in Küttel et al., 2002) selon les auteurs et les températures d'acclimatation. Kraiem \& Pattee (1980) ont également mesuré une CL50 pour le dioxygène de $1,8 \mathrm{mg} . \mathrm{I}^{-1}$ à $20^{\circ} \mathrm{C}(\mathrm{CL}$ sur 24 heures de $1,9 \mathrm{mg} . \mathrm{I}^{-1}$ ) et une $\mathrm{CT} \max$ de $30^{\circ} \mathrm{C}$ à $20^{\circ} \mathrm{C}$. Cette température imite paraît faible au regard des optimums mentionnés dans les autres références. Elle n'a donc pas été retenue comme température extrême supportée.

Quelques données sur les températures de début de reproduction sont mentionnées, notamment via des articles de synthèse (Alabaster \& Lloyd, 1982; Kennedy \& Fitzmaurice, 1972) : l'optimum minimal s'établit à $12{ }^{\circ} \mathrm{C}$ (Elliott, 1981; Kryzhanovskii, 1949 in Alabaster \& Lloyd, 1982; Mann, 1996) et le maximum autour de $17{ }^{\circ} \mathrm{C}$ (Bourgeois, 1964 in Kennedy \& Fitzmaurice, 1972; Mann, 1996). Banarescu (2003b) mentionne une température extrême maximale pour la reproduction à $24^{\circ} \mathrm{C}$.

\subsubsection{Le chevesne (Leuciscus cephalus)}

Pour l'embryon de chevesne, Elliott (1981 in Küttel et al., 2002) et Alabaster \& Lloyd (1982) observent une température optimale inférieure à $16{ }^{\circ} \mathrm{C}$, l'optimum supérieur étant de $24{ }^{\circ} \mathrm{C}$ pour Alabaster \& Lloyd (1982) sur un lac réchauffé. Ces deux auteurs s'accordent sur une température maximale supportée à $30{ }^{\circ} \mathrm{C}$ (Alabaster \& Lloyd, 1982 observent cette valeur in situ), cohérente avec celle de $29{ }^{\circ} \mathrm{C}$ en laboratoire, annoncée par Mamcarz et al. (2005). La température létale inférieure en laboratoire est à $9,5{ }^{\circ} \mathrm{C}$ pour Mamcarz et al. (2005). Dix données concernant la durée séparant la ponte de l'éclosion sont mentionnées par sept auteurs : elles s'échelonnent entre 1,5 jours à $27,5^{\circ} \mathrm{C}$ (Kupren et al., 2008) et 9 jours à $12,3^{\circ} \mathrm{C}$ (Mamcarz et al., 2005). 
La gamme de température optimale pour la larve est située entre $14{ }^{\circ} \mathrm{C}$ (Penaz \& Sterba, 1969) et $25{ }^{\circ} \mathrm{C}$ (Kwiatkowski et al., 2008; Wolnicki \& Myszkowski, 1999), avec trois références répertoriées sur cette plage (Shiri Harzevili et al., 2003; Teletchea et al., 2008; Fishbase). L'ensemble des données pour établir cette gamme est issu d'expérimentations en laboratoire. Philippart et al. (1989) évaluent à 150 degrés jours le temps écoulé entre la ponte et la sortie du stade larvaire, valeur bien inférieure aux 21 jours à $25{ }^{\circ} \mathrm{C}$ avancés par deux auteurs (Kwiatkowski et al., 2008 ; Zarski et al., 2008).

La gamme optimale pour le juvénile est prise semblable à celle de la larve, qui recoupe bien les tolérances mentionnées sur le Rhône (Ginot et al., 1996). L'extrême maximum supérieur est choisi identique à celui de l'adulte $\left(30{ }^{\circ} \mathrm{C}\right)$.

La gamme optimale des températures pour le stade adulte est estimée entre $14{ }^{\circ} \mathrm{C}$ et $24{ }^{\circ} \mathrm{C}$ (Cowx \& Welcomme, 1998; Ginot et al., 1996 sur le Rhône). La température extrême minimale est située autour $8{ }^{\circ} \mathrm{C}$ et l'extrême maximal à $30{ }^{\circ} \mathrm{C}$ (Alabaster \& Lloyd, 1982; Elliott, 1981). Deux observations menées par Horoszewicz (1973 in Alabaster \& Lloyd, 1982) sur des retenues polonaises qui s'échauffent de $6{ }^{\circ} \mathrm{C}$ par heure permettent d'établir une température létale à $37,9^{\circ} \mathrm{C}$ (acclimatation à $\left.25,7^{\circ} \mathrm{C}\right)$ et à $38,8^{\circ} \mathrm{C}$ (acclimatation à $\left.30,8^{\circ} \mathrm{C}\right)$.

Les températures optimales de reproduction se situent entre $15^{\circ} \mathrm{C}$ et $20{ }^{\circ} \mathrm{C}$ (Kucharczyk et al., 2008 en laboratoire).

\subsubsection{La vandoise (Leuciscus leuciscus)}

Le stade embryonnaire de la vandoise est largement renseigné (Bruslé \& Quignard, 2001; Cowx \& Welcomme, 1998; Herzig \& Winkler, 1985 in Küttel et al., 2002; Kennedy, 1969 in Wüstemann \& Kammerad, 1995; Kupren et al., 2008; Mamcarz et al., 2005; Mills, 1980; Teletchea et al., 2008; Wurtz-Arlet, 1950). En laboratoire, l'embryon de vandoise ne semble pas pouvoir survivre à moins de $4{ }^{\circ} \mathrm{C}$ (Herzig \& Winkler, 1985 in Küttel et al., 2002; Mamcarz et al., 2005 en laboratoire; Mills, 1980 en laboratoire), sa gamme de température optimale étant 7-15 ${ }^{\circ} \mathrm{C}$ (Mamcarz et al., 2005 ; Mills, 1991 in Küttel et al., 2002), avec un optimum maximal à $23^{\circ} \mathrm{C}$ et une température létale estimée à $25^{\circ} \mathrm{C}$ par Mamcarz et al. (2005) en laboratoire. À noter toutefois que Mills (1980) signale un arrêt de la croissance à $16,5^{\circ} \mathrm{C}$ en laboratoire.

Le temps d'incubation est résumé dans l'équation de Mills (1980), à partir d'expériences en laboratoire :

$$
D=96,59-9,3 T+0,254 T^{2}
$$

soit, 46 jours à $6,75{ }^{\circ} \mathrm{C}, 32$ jours à $8,8^{\circ} \mathrm{C}, 30$ jours à $10^{\circ} \mathrm{C}, 12$ jours à $16,5^{\circ} \mathrm{C}$.

Pour la larve la plage de température optimale semble être comprise entre $16{ }^{\circ} \mathrm{C}$ (Lepičová et al., 2002) et $25{ }^{\circ} \mathrm{C}$ (Herzig \& Winkler, 1985; Kwiatkowski et al., 2008). Teletchea et al. (2008) signalent une température extrême inférieure de $12^{\circ} \mathrm{C}$.

Pour le juvénile et l'adulte, Wüstemann \& Kammerad (1995) donnent des températures tolérées entre $4^{\circ} \mathrm{C}$ et $22^{\circ} \mathrm{C}$, une plage optimale 
entre $10^{\circ} \mathrm{C}$ et $20^{\circ} \mathrm{C}$ et une température létale à $32{ }^{\circ} \mathrm{C}$. Cette température létale dépend de la concentration en oxygène dissous, les mêmes auteurs (Wüstemann \& Kammerad, 1995) ont ainsi mesuré pour l'adulte de vandoise une $\mathrm{CL}$ sur 24 heures à $20^{\circ} \mathrm{C}$ pour le dioxygène de $3,2 \mathrm{mg} . \mathrm{I}^{-1}$ (la vandoise ne survit pas à long terme à cette concentration). La gamme des températures optimales pour le juvénile est prise identique à la gamme élargie de la larve (12-25 $\left.{ }^{\circ} \mathrm{C}\right)$. L'optimum maximal pour l'adulte est également pris à $25^{\circ} \mathrm{C}$.

La reproduction de la vandoise s'effectue préférentiellement dans la plage $7{ }^{\circ} \mathrm{C}$ (Mann, 1974; Millet, 1989; Philippart, 1981) à $12{ }^{\circ} \mathrm{C}$ (Kucharczyk et al., 2008 ; Mann, 1996), avec sept références mentionnant des températures optimales entre ces limites (Bourgeois, 1963 en France, in Wüstemann \& Kammerad, 1995; Herzig \& Winkler, 1985 en laboratoire, in Küttel et al., 2002 ; Kennedy, 1969; Lelek, 1987; Philippart et al., 1989; Poncin, 1996 sur la Meuse; Teletchea et al., 2008). Elle resterait possible entre $5{ }^{\circ} \mathrm{C}$ (Alabaster \& Lloyd, 1982; Kucharczyk et al., 1998; Mann, 1996) et $16,5^{\circ} \mathrm{C}$ (Mills, 1980 in Millet, 1989 en laboratoire).

\subsubsection{Le spirlin (Alburnoides bipunctatus)}

La durée d'incubation du spirlin se situe autour de 100 degrés jours (Bless, 1996 qui mesure 5,2 jours à 19,3 ${ }^{\circ} \mathrm{C}$; Bohl et al., 2004 ; Spillmann, 1961).

La gamme de température optimale pour le stade larvaire est située entre
$19{ }^{\circ} \mathrm{C}$ et $24{ }^{\circ} \mathrm{C}$ (Alabaster \& Lloyd, 1982), $12{ }^{\circ} \mathrm{C}$ étant la température extrême inférieure (Bless, 1996).

La gamme des températures optimales étendue de la larve est retenue pour l'optimum du juvénile $\left(12-24^{\circ} \mathrm{C}\right)$.

Seules des températures extrêmes sont mentionnées pour l'adulte de spirlin : elles se répartissent entre $1,9{ }^{\circ} \mathrm{C}$ et $24{ }^{\circ} \mathrm{C}$ (Bohl et al., 2004), avec une température létale autour de $27^{\circ} \mathrm{C}$ in situ en Suisse (Breitenstein \& Kirchhofer, 1999). La gamme des températures optimales semble donc se situer, tout comme la larve et le juvénile, entre $12{ }^{\circ} \mathrm{C}$ (optimum minimal retenu pour le juvénile sur la base de l'extrême minimum de la larve) et $24^{\circ} \mathrm{C}$.

Quelques références font état de températures optimales pour la reproduction : la plage est comprise entre $14{ }^{\circ} \mathrm{C}$ (Mann, 1996 in Breitenstein \& Kirchhofer, 1999; Pelz, 1986 in Breitenstein \& Kirchhofer, 1999) et $21{ }^{\circ} \mathrm{C}$ (Bohl et al., 2004), avec deux références mentionnant des optimums dans cette gamme (Holcik \& Hensel, 1971 in Papadopol \& Cristofor, 1980 ; Philippart et al., 1989). Les températures extrêmes permettant la reproduction sont observées entre $12{ }^{\circ} \mathrm{C}$ (Bless, 1996 en laboratoire; Keith \& Allardi, 2001) et $25^{\circ} \mathrm{C}$ (Yildirim et al., 1999 en Turquie). Le spirlin est un poisson à ponte fractionnée, ce qui explique l'étendue de la plage de température où il peut se reproduire.

\subsubsection{Le barbeau fluviatile (Barbus barbus)}

Les tolérances thermiques de l'embryon de barbeau sont largement documentées (notamment grâce aux 
synthèses de Kamler, 2002 ; Krupka, 1988; Küttel et al., 2002). L'optimum de température se situe pour la plupart des auteurs entre $16{ }^{\circ} \mathrm{C}$ et $21^{\circ} \mathrm{C}$ (Herzig \& Winkler, 1985 in Küttel et al., 2002 ; Kraiem \& Pattee, 1980 ; Penaz, 1973 in Kamler, 2002). Seul Baras (1995 in Bruslé \& Quignard, 2001) note un optimum plus bas, à $13{ }^{\circ} \mathrm{C}$. Penaz (1973 in Kamler, 2002) observe l'arrêt de la croissance en dessous de $12{ }^{\circ} \mathrm{C}$ et Elliott (1981 in Küttel et al., 2002) au-dessus de $20{ }^{\circ} \mathrm{C}$. Krupka (1988) répertorie six durées entre la ponte et l'éclosion, issues de différentes études (Gyurko et al., 1957 en Roumanie; Krupka, 1988; Penaz, 1973) : elles sont comprises entre 3,7 jours (Krupka, 1988) et 8 jours (Penaz, 1973) pour des températures de $20,2{ }^{\circ} \mathrm{C}$ à $16{ }^{\circ} \mathrm{C}$ respectivement. Ses valeurs sont relativement bien recoupées avec celles annoncées par Cowx \& Welcomme (1998) (11 jours à $14^{\circ} \mathrm{C}$ et $5-6$ jours à $\left.19^{\circ} \mathrm{C}\right)$, par Poncin (1996) sur la Meuse en Belgique (3-4 jours à $20{ }^{\circ} \mathrm{C}$ ) et par Telechea et al. (2008) en laboratoire (5,6 jours à $17,5^{\circ} \mathrm{C}$ ). Ces valeurs ne permettent pas d'évaluer la durée entre la ponte et l'éclosion des larves de barbeau sous forme de degrés jours, sur la plage de température étudiée par ces auteurs $\left(14-20,5^{\circ} \mathrm{C}\right)$.

Les différents auteurs, qui ont étudié le stade larvaire en laboratoire, élèvent les larves de barbeau entre $14,8{ }^{\circ} \mathrm{C}$ et $18,9{ }^{\circ} \mathrm{C}$ (Teletchea et al., 2008; Vladimirov \& Bodareu, 1975 in Banarescu, 2003a). La température extrême supportée en laboratoire peut atteindre $24,5{ }^{\circ} \mathrm{C}$ (Bodareu \& Karlov, 1984 in Banarescu, 2003a). Krupka (1988) estime à 12,6 jours à $20,2{ }^{\circ} \mathrm{C}$ la durée du stade larvaire.
Baras \& Philippart (1999) ont montré que la croissance du juvénile du barbeau s'arrête en dessous de $13,5^{\circ} \mathrm{C}$ sur l'Ourthe en Belgique. Par ailleurs, Ginot et al. (1996) évaluent la plage de température optimale sur le Rhône entre $7{ }^{\circ} \mathrm{C}$ et $27^{\circ} \mathrm{C}$. La gamme des températures optimales semble donc s'établir entre $13{ }^{\circ} \mathrm{C}$ et $24^{\circ} \mathrm{C}\left(24^{\circ} \mathrm{C}\right.$ correspondant à l'extrême supérieur de la larve). Kraiem \& Pattee (1980) ont fait plusieurs expérimentations en laboratoire sur des juvéniles de barbeau qui ont permis d'évaluer : une CT maximum de $31,5{ }^{\circ} \mathrm{C}$ (choisie en extrême maximal supporté par le stade) et une IULT sur 24 heures de $30{ }^{\circ} \mathrm{C}$ pour une acclimatation de 10 jours à $20{ }^{\circ} \mathrm{C}$ (à cette température, ils mesurent une $\mathrm{CL}$ pour le dioxygène de $2,1 \mathrm{mg} . \mathrm{I}^{-1}$ sur 24 heures, la CL50 est de $2 \mathrm{mg}^{-1} \mathrm{I}^{-1}$ ) ainsi qu'une IULT sur 24 heures de $29,7{ }^{\circ} \mathrm{C}$ pour une acclimatation à $16{ }^{\circ} \mathrm{C}$.

Les températures optimales pour le stade adulte s'échelonnent entre $10{ }^{\circ} \mathrm{C}$ et $24^{\circ} \mathrm{C}$ (Ginot et al., 1996 sur le Rhône; Leynaud \& Allardi, 1974 sur la Seine; Fishbase). La température maximale tolérée est choisie à $32{ }^{\circ} \mathrm{C}$, par analogie avec le juvénile (Kraiem \& Pattee, 1980).

Le barbeau se reproduit préférentiellement à des températures comprises entre $13,5{ }^{\circ} \mathrm{C}$ (Baras \& Philippart, 1999 ; Elliott, 1981 in Küttel et al., 2002; Prignon et al., 1988) et $20{ }^{\circ} \mathrm{C}$ (Elliott, 1981 in Küttel et al., 2002 ; Mann, 1996), même si sa reproduction peut démarrer à partir de $8{ }^{\circ} \mathrm{C}$ (1985 in Küttel et al., 2002) et se poursuivre jusqu'à $25^{\circ} \mathrm{C}$ (Banarescu et al., 2003a) et même $29^{\circ} \mathrm{C}$ (Alabaster \& Lloyd, 1982). 


\subsubsection{La bouvière (Rhodeus sericeus)}

La plage de température optimale pour l'adulte de bouvière est estimée entre $12{ }^{\circ} \mathrm{C}$ et $24,3^{\circ} \mathrm{C}$ par Wohlgemuth (1981) et jusqu'à $30^{\circ} \mathrm{C}$ par Zahn (1963 in Jobling, 1981). La température létale dans un lac réchauffé en Pologne est de $37^{\circ} \mathrm{C}$ (Horosewicz, 1971 in Jobling, 1981).

Dans différentes synthèses qui mentionnent Duyvené de Wit (1955), l'optimum pour la reproduction de la bouvière se situe entre $15^{\circ} \mathrm{C}$ et $21^{\circ} \mathrm{C}$ (Holcik, 2003; fiche Natura 2000) avec des extrêmes entre $12{ }^{\circ} \mathrm{C}$ et $26,2{ }^{\circ} \mathrm{C}$ (Konečná et al., 2009; Tarkan et al., 2005). Bruslé \& Quignard (2001) précisent quant à eux que la température de l'eau doit être dans la gamme 13-22 ${ }^{\circ} \mathrm{C}$ pour que la gamétogenèse ait lieu.

\subsubsection{Le gardon (Rutilus rutilus)}

Pour le stade embryonnaire du gardon, Gulidov \& Popova (1981 in Gillet, 1989) observent la gamme des températures optimales entre $12^{\circ} \mathrm{C}$ et $24^{\circ} \mathrm{C}$, la température létale est quant à elle estimée à $26^{\circ} \mathrm{C}$ par Horosewicz (1971 in Nzau Matondo et al., 2007) dans un lac réchauffé en Pologne. Teletchea et al. (2008) citent une durée entre la ponte et l'éclosion de la larve de 7,4 jours à $15,5{ }^{\circ} \mathrm{C}$ et Cowx \& Welcomme (1998) de 11 à 12 jours à $12,9^{\circ} \mathrm{C}$ et de 30 jours à $7{ }^{\circ} \mathrm{C}$.

Le site Fishbase et Teletchea et al. (2008) citent une même valeur de température optimale pour le stade larvaire du gardon : $17,5^{\circ} \mathrm{C}$.
Ginot et al. (1996) observent des températures optimales pour les juvéniles entre $7^{\circ} \mathrm{C}$ et $21^{\circ} \mathrm{C}$ sur le Rhône. Barbier \& Pascal (1976) ont réalisé des expériences sur des juvéniles de gardon, pour neuf températures d'acclimatation (de $14,2{ }^{\circ} \mathrm{C}$ à $34^{\circ} \mathrm{C}$ ). Ils ont obtenu des IULT sur 24 heures comprises entre $26,9^{\circ} \mathrm{C}$ et $34,7^{\circ} \mathrm{C}$, la plupart autour de $30{ }^{\circ} \mathrm{C}$ qui est considéré comme l'extrême supérieur maximum de tolérance.

Pour son stade adulte, les températures optimales s'étalent sur une très large gamme pour Elliott (1994) : $12{ }^{\circ} \mathrm{C}$ pour le minimum et $25^{\circ} \mathrm{C}$ pour le maximum. Deux auteurs ont complété ces températures optimales par des expériences en laboratoire menées en Angleterre. Pour des températures d'acclimatation de $15^{\circ} \mathrm{C}, 20^{\circ} \mathrm{C}$ et $25^{\circ} \mathrm{C}$ Alabaster (1964) mesure des IULT sur 1000 minutes de $27,3{ }^{\circ} \mathrm{C}, 29,4{ }^{\circ} \mathrm{C}$ et $31,5{ }^{\circ} \mathrm{C}$ respectivement. Cocking (1959) a noté les températures létales supérieures comprises entre $30,1{ }^{\circ} \mathrm{C}$ et $35,2{ }^{\circ} \mathrm{C}$ au cours de sept expériences (acclimatation variant de $17^{\circ} \mathrm{C}$ à $33^{\circ} \mathrm{C}$ ) ainsi que l'ILLT la plus faible, à $7{ }^{\circ} \mathrm{C}$, pour une acclimatation à $23^{\circ} \mathrm{C}$. L'extrême supérieur de tolérance est choisi à $31{ }^{\circ} \mathrm{C}$ d'après Cocking (1959) (IULT maximum à $20^{\circ} \mathrm{C}$ ).

La température optimale pour la reproduction du gardon se situe entre $10{ }^{\circ} \mathrm{C}$ (Easton \& Dolben, 1980 in Gillet \& Quétin, 2006; L’Abbée-Lund \& Vøllestad, 1985 in Gillet, 1989 en Scandinavie) et $18{ }^{\circ} \mathrm{C}$ (Holcik \& Hruska, 1966 in Gillet \& Quétin, 2006), gamme confirmée par sept références d'optimum (Diamond, 1985 en Grande Bretagne in Gillet \& Quétin, 2006 ; Easton \& Dolben, 1980 in Gillet, 1989; Elliott, 1994; Mills, 1981 in 
Gillet, 1989 ; Poncin, 1996 ; Spillmann, 1961; Teletchea et al., 2008). La température extrême inférieure est mentionnée autour de $7{ }^{\circ} \mathrm{C}$ (Mann, 1996; Noges \& Jarvet, 2005 en Estonie) et la température extrême supérieure autour de $22^{\circ} \mathrm{C}$ (Gillet \& Quétin, 2006 sur le lac Léman).

\subsubsection{L'ablette (Alburnus alburnus)}

Seuls Alabaster \& Lloyd (1982) ont étudié le stade embryonnaire de l'ablette en calculant une CT minimum de $14{ }^{\circ} \mathrm{C}$ et une CT maximum de $31{ }^{\circ} \mathrm{C}$ (limites de la gamme élargie des températures maximales et minimales supportées) pour une gamme de température optimale de $21{ }^{\circ} \mathrm{C}$ à $27^{\circ} \mathrm{C}$. Spillman (1961) estime que 14 à 21 jours sont nécessaires entre la ponte et l'éclosion de la larve.

D'après le site Fishbase, la température optimale pour la larve d'ablette est située autour de $22,5^{\circ} \mathrm{C}$.

Pour le stade adulte, l'optimum est incertain : Elliott (1981 in Küttel et al., 2002) mentionne une température de $30{ }^{\circ} \mathrm{C}$, tandis qu'il est situé autour de $20{ }^{\circ} \mathrm{C}$ pour Alabaster \& Downing (1966 in Coutant, 1975). La température létale est quant à elle observée à $37,7^{\circ} \mathrm{C}$ pour une acclimatation à $25,7-26,2^{\circ} \mathrm{C}$ par Horoszewicz (1973 in Alabaster \& Lloyd, 1982) dans une retenue soumise à un réchauffement de $6{ }^{\circ} \mathrm{C}$ par heure en Pologne. Le maximum tolérable est donc vraisemblablement légèrement inférieur, sans doute autour de $35^{\circ} \mathrm{C}$.

Plusieurs références, à la fois des compilations de données (Keith \& Allardi, 2001; Prokes \& Penaz, 1978) mais aussi des études in situ (Berg, 1949 ; Chappaz et al., 1987 sur le lac de Sainte Croix), situent le début de la reproduction à $15{ }^{\circ} \mathrm{C}$ et tous les auteurs donnent un optimum maximal à $28{ }^{\circ} \mathrm{C}$ (Alabaster \& Lloyd, 1982 ; Keith \& Allardi, 2001; Mann, 1996; Statova, 1973 in Gillet, 1989). Le minimum toléré pour la reproduction est à $14{ }^{\circ} \mathrm{C}$ (Alabaster \& Lloyd, 1982).

\subsubsection{La brème commune (Abramis brama)}

La gamme de température optimale pour l'embryon de brème commune est située entre $12{ }^{\circ} \mathrm{C}$ (Herzig \& Winkler, 1986 in Küttel et al., 2002) et $23{ }^{\circ} \mathrm{C}$ (Alabaster \& Lloyd, 1982). L'extrême inférieur est observé autour de 8-9 ${ }^{\circ} \mathrm{C}$ (Alabaster \& Lloyd, 1982 ; Elliott, 1981 in Küttel et al., 2002) tandis que la température létale inférieure est mesurée en laboratoire à $5{ }^{\circ} \mathrm{C}$ (Herzig \& Winkler, 1986 in Küttel et al., 2002). L'extrême supérieur est mentionné à $28{ }^{\circ} \mathrm{C}$ (Elliott, 1981 in Küttel et al., 2002) avec une température létale à $32{ }^{\circ} \mathrm{C}$ (Herzig \& Winkler, 1986 in Küttel et al., 2002). La durée entre la ponte et l'éclosion est évaluée à 9 jours à $20{ }^{\circ} \mathrm{C}$ et 10 jours à $18^{\circ} \mathrm{C}$ pour Bruslé \& Quignard (2001) contre 5,8 jours à $20{ }^{\circ} \mathrm{C}$ pour Keith \& Allardi (2001) et 7,5 jours à $18^{\circ} \mathrm{C}$ pour Cowx \& Welcomme (1998). À $16,5^{\circ} \mathrm{C}$, Telechea et al. (2008) l'estiment quant à eux autour de 6,4 jours.

D'après le site Fishbase, la température optimale pour la larve est de $17,5^{\circ} \mathrm{C}$. Telechea et al. (2008) l'établissent quant à eux à $19,5^{\circ} \mathrm{C}$.

Pour le stade juvénile, l'optimum élargi se trouve entre $14{ }^{\circ} \mathrm{C}$ et $34^{\circ} \mathrm{C}$ (Kucharczyk et al., 1998 in Küttel et al., 2002). Nous considérons que l'optimum maximal semble se situer à 
des températures moindres, autour de $28{ }^{\circ} \mathrm{C}$.

La gamme optimale pour la vie adulte s'échelonne entre $10^{\circ} \mathrm{C}$ (site Fishbase) et $26{ }^{\circ} \mathrm{C}$ (Müller, 1997 in Küttel et al., 2002), avec des températures extrêmes tolérées entre $5{ }^{\circ} \mathrm{C}$ (Holker, 2006) et $28^{\circ} \mathrm{C}$ (EIFAC, 1969 in Küttel et al., 2002). La température létale supérieure est observée à $35,7^{\circ} \mathrm{C}$ pour une acclimatation à $25,7{ }^{\circ} \mathrm{C}$ dans un lac réchauffé en Pologne (Horoszewicz, 1972). Plusieurs expériences en laboratoire mettent également en évidence des températures limites. Alabaster (1964) a mesuré une IULT sur 1000 minutes de $30,2{ }^{\circ} \mathrm{C}$ après une acclimatation à $20{ }^{\circ} \mathrm{C}$. Dans la synthèse établie par Küttel et al. (2002), des CT maximum de $35{ }^{\circ} \mathrm{C}$ (Müller, 1997), choisie en extrême maximal supporté et $36{ }^{\circ} \mathrm{C}$ (EIFAC, 1969) sont mentionnées. Enfin Alabaster \& Downing (1966) mesurent une CT maximum de $30,2{ }^{\circ} \mathrm{C}$ pour une acclimatation à $20^{\circ} \mathrm{C}$.

La gamme optimale pour la reproduction de la brème commune est située entre $12{ }^{\circ} \mathrm{C}$ (Bruslé \& Quignard, 2001; Löffler, 1982; Mann, 1996) et $20{ }^{\circ} \mathrm{C}$ (Herzig \& Winkler, 1985 in Küttel et al., 2002; Keith \& Allardi, 2001; Mann, 1996; Poncin, 1996 sur la Meuse). La température extrême inférieure est estimée autour de $8{ }^{\circ} \mathrm{C}$ (EIFAC, 1969 in Küttel et al., 2002) et l'extrême supérieur à $28{ }^{\circ} \mathrm{C}$ (Statova, 1973 in Gillet, 1989).

\subsubsection{La brème bordelière (Blicca bjoerkna)}

Pour le stade embryonnaire de la brème bordelière, la seule donnée recueillie est celle mentionnée par Cowx \& Welcomme (1998) qui estiment à 4 jours à $20^{\circ} \mathrm{C}$ (et 5 jours à $15^{\circ} \mathrm{C}$ ) le temps écoulé entre la ponte et l'éclosion de la larve.

Les observations sur le Rhône mettent en évidence la présence de juvéniles de brème bordelière entre $4{ }^{\circ} \mathrm{C}$ et $27^{\circ} \mathrm{C}$ (Ginot et al., 1996).

La gamme optimale pour le stade adulte est évaluée entre $16{ }^{\circ} \mathrm{C}$ et $25{ }^{\circ} \mathrm{C}$ par Cowx \& Welcomme (1998). Brylinska \& Zbikowska (1997) mentionnent également l'optimum adulte dans cette plage. Sur le Rhône à Bugey, Ginot et al. (1996) observent des températures tolérées de $4{ }^{\circ} \mathrm{C}$ à $27^{\circ} \mathrm{C}$

L'optimum de reproduction est situé entre $15{ }^{\circ} \mathrm{C}$ (Poncin, 1996 sur la Meuse) et $25^{\circ} \mathrm{C}$ (Mann, 1996), avec deux auteurs situant l'optimum dans cette gamme (Brylinska \& Zbikowska, 1997; Peczalska, 1979 in Brylinska \& Zbikowska, 1997). La reproduction peut néanmoins s'étendre de $9,6{ }^{\circ} \mathrm{C}$ à $29{ }^{\circ} \mathrm{C}$ en Russie (Roenko, 1965 in Brylinska \& Zbikowska, 1997).

\subsection{Famille des Percidae}

\subsubsection{La perche fluviatile (Perca fluviatilis)}

L'embryon de perche fluviatile est bien documenté : la gamme large des températures optimales s'observe entre $7{ }^{\circ} \mathrm{C}$ (Gillet, 1989) et $21{ }^{\circ} \mathrm{C}$ (Hokanson \& Kleiner, 1974; Wang \& Eckmann, 1994 in Küttel et al., 2002) et la gamme plus restreinte entre $12{ }^{\circ} \mathrm{C}$ (Alabaster \& Lloyd, 1982; Wang \& Eckmann, 1994 in Küttel et al., 2002) et $18{ }^{\circ} \mathrm{C}$ (Alabaster \& Lloyd, 1982; Saat \& Veersalu, 1996). Cinq références 
mentionnent des températures limites obtenues en laboratoire (Alabaster \& Lloyd, 1982; Elliott, 1981 in Küttel et al., 2002 ; Hokanson, 1977 in Karas, 1996 ; Swift, 1965 in Küttel et al., 2002 ; Wang \& Eckmann, 1994 in Küttel et al., 2002) : la valeur d'ILLT minimale est de $6{ }^{\circ} \mathrm{C}$ (Elliott, 1981 ; Hokanson, 1977 in Karas, 1996; Wang \& Eckmann, 1994 in Küttel et al., 2002) tandis que la maximale est mesurée à $26{ }^{\circ} \mathrm{C}$ (Alabaster \& Lloyd, 1982). Une durée de 13,2 jours à $12,5{ }^{\circ} \mathrm{C}$ est constatée entre la ponte et l'éclosion de la larve en laboratoire par Teletchea et al. (2008).

Pour la larve, l'optimum de température est compris entre $12^{\circ} \mathrm{C}$ (EIFAC, 1969 in Küttel et al., 2002) et $25^{\circ} \mathrm{C}$ (Karas, 1996). L'extrême minimal est évalué autour de $5{ }^{\circ} \mathrm{C}$, un autre auteur indiquant des températures optimales dans cette gamme large (Karas, 1987 in Urho, 1996). Hokanson (1977 in Karas, 1996) cite une IULT à $36{ }^{\circ} \mathrm{C}$ et une $\mathrm{CT}$ maximum à $34{ }^{\circ} \mathrm{C}$, choisie en extrême maximal supporté. Keith \& Allardi (2001) évaluent entre 120 et 200 degrés jours la durée du stade larvaire.

Le stade juvénile a été essentiellement étudié via des expériences en laboratoire et seuls deux références font état de températures optimales : Karas (1990) observe des températures d'arrêt de croissance en dessous du seuil de $8-10^{\circ} \mathrm{C}$ et Hokanson \& Kleiner (1974) mentionnent un optimum à $25^{\circ} \mathrm{C}$. La synthèse d'Hokanson (1977) cite des résultats de CT maximum autour de $35,5^{\circ} \mathrm{C}$ (Weatherley, 1973) et $35,9^{\circ} \mathrm{C}$ (Horoszewicz, 1973) ainsi que des IULT comprises entre $31,4^{\circ} \mathrm{C}$ (Alabaster \& Downing, 1966) et $33,5^{\circ} \mathrm{C}$ (Willemsen, non publié) pour une température d'acclimatation entre $25{ }^{\circ} \mathrm{C}$ et $30{ }^{\circ} \mathrm{C}$. Ces valeurs d'IULT paraissent davantage cohérentes avec la gamme optimale choisie, la valeur de $32{ }^{\circ} \mathrm{C}$ est donc préférée à celle de $35{ }^{\circ} \mathrm{C}$ des CT max pour représenter le maximum supérieur supporté par le stade.

Pour l'adulte, de nombreux auteurs ont répertorié des températures limites. La gamme optimale semble évoluer entre $16^{\circ} \mathrm{C}$ et $27^{\circ} \mathrm{C}$ (Craig, 1978 en Angleterre; Horoszewicz, 1973 in Hokanson, 1977). Une gamme étendue des températures optimales existe entre $12{ }^{\circ} \mathrm{C}$ et $31{ }^{\circ} \mathrm{C}$ en Amérique du nord et en Australie où l'espèce a été introduite (Thorpe, 1977b). Keith \& Allardi (2001) mentionnent un arrêt de croissance autour de $10{ }^{\circ} \mathrm{C}$. Enfin, trois auteurs donnent des températures létales in situ selon la température d'acclimatation : Shkorbatov (1964 in Alabaster \& Lloyd, 1982) observe des mortalités à $30^{\circ} \mathrm{C}$ et $33,5^{\circ} \mathrm{C}$ en Russie, Filon (1972 in Alabaster \& Lloyd, 1982) note quant à lui des températures létales de $23,2^{\circ} \mathrm{C}$ (pour une acclimatation à $0,1^{\circ} \mathrm{C}$ ) et $36,2^{\circ} \mathrm{C}$ (pour une acclimatation $28,4^{\circ} \mathrm{C}$ ) également en Russie, comparables à celles observées en Pologne par Horoszewicz (1973 in Alabaster \& Lloyd, 1982). Des valeurs de températures limites établies en laboratoire (IULT et CT maximum) sont avancées par sept auteurs. Willemsen (1977 in Alabaster \& Lloyd, 1982) et EIFAC (1969 in Küttel et al., 2002) donnent des CT maximum de $33^{\circ} \mathrm{C}$ et $34{ }^{\circ} \mathrm{C}$ respectivement (pris en température maximale supérieure supportée par le stade). Colette et al. (1977) en Iran, Müller (1997 in Küttel et al., 2002), Thorpe (1977b) et Varley (1967 in Küttel et al., 2002) 
mesurent quant à eux des valeurs d'IULT sur une durée non précisée comprises entre $30{ }^{\circ} \mathrm{C}$ et $33,5^{\circ} \mathrm{C}$. Sur 1000 minutes et pour des températures d'acclimatation comprises entre $6{ }^{\circ} \mathrm{C}$ et $25^{\circ} \mathrm{C}$, Alabaster (1964) obtient des IULT entre $24{ }^{\circ} \mathrm{C}$ et $31,4^{\circ} \mathrm{C}$.

Dans sa synthèse, Hokanson (1977) propose une gamme de température optimale pour la reproduction entre $5^{\circ} \mathrm{C}$ et $19{ }^{\circ} \mathrm{C}$ selon les régions (Bastl, 1969; EIFAC, 1968 ; Kovalev, 1973), qui correspond à la gamme large retrouvée dans la littérature (Alabaster \& Lloyd, 1982; Elliott, 1981 in Küttel et al., 2002; Poncin, 1996 sur la Meuse; Thorpe, 1977a). La gamme restreinte s'établit entre $8^{\circ} \mathrm{C}$ et $15^{\circ} \mathrm{C}$ (Gillet, 1989; Gillet \& Dubois, 2007 sur le lac Léman; Lam, 1983; Mann, 1996 ; Philippart et al., 1989 ; Teletchea et al., 2008; site Fishbase).

\subsubsection{La grémille (Gymnocephalus cernuus)}

L'optimum thermique pour l'embryon de grémille est situé entre $9{ }^{\circ} \mathrm{C}$ et $21^{\circ} \mathrm{C}$ en laboratoire (Saat \& Veersalu, 1996) et les maxima tolérés sont compris entre $6{ }^{\circ} \mathrm{C}$ et $24^{\circ} \mathrm{C}$ en laboratoire (Saat \& Veersalu, 1996). Les durées d'incubation mentionnées par Bruslé \& Quignard (2001), Teletchea \& Fostier (2008) et Ogle (1998) sont cohérentes : 10 jours à $12,5^{\circ} \mathrm{C}$, 8 jours à $14,5^{\circ} \mathrm{C}$ et entre 5 et 12 jours à $10-15^{\circ} \mathrm{C}$ respectivement. Fairchild \& McCormick (1996) trouvent par contre des durées nettement plus longues pour l'incubation de la grémille : 9, 14 et 26 jours à $11^{\circ} \mathrm{C}, 16^{\circ} \mathrm{C}$ et $21^{\circ} \mathrm{C}$ respectivement.
La plage optimale pour la larve est située entre $25^{\circ} \mathrm{C}$ et $30^{\circ} \mathrm{C}$ (Kammerer, 1907), la température minimale tolérée étant de $10{ }^{\circ} \mathrm{C}$ (Hokanson, 1977 in Ogle, 1998).

Le juvénile de grémille croît entre $7{ }^{\circ} \mathrm{C}$ et $24,8{ }^{\circ} \mathrm{C}$, avec un optimum à $21{ }^{\circ} \mathrm{C}$ (Edsall et al., 1993 in Ogle, 1998). Hokanson (1977) répertorie deux mesures de température limite en laboratoire : une IULT sur 1000 minutes de $30,4{ }^{\circ} \mathrm{C}$ après une acclimatation de 11 jours à $20{ }^{\circ} \mathrm{C}$ (Alabaster \& Downing, 1966) et une CT maximum de $34,5^{\circ} \mathrm{C}$ après une acclimatation à 24,1-25,7 ${ }^{\circ} \mathrm{C}$ (Horoszewicz, 1973). Le maximum toléré par le juvénile de grémille est pris à $30{ }^{\circ} \mathrm{C}$, la $\mathrm{CT}$ max mesurée l'étant à une température d'acclimatation en limite d'optimum du stade.

Alabaster (1964) a mené des tests en laboratoire sur des adultes à $15^{\circ} \mathrm{C}$ et $20^{\circ} \mathrm{C}$ : il mesure respectivement des IULT sur 1000 minutes de $28,1^{\circ} \mathrm{C}$ et $30,4{ }^{\circ} \mathrm{C}$. La gamme optimale est choisie entre $15^{\circ} \mathrm{C}$ et $25^{\circ} \mathrm{C}$ (température optimale maximale retenue pour le juvénile), avec un maximum à $31^{\circ} \mathrm{C}$ (Alabaster, 1964).

La reproduction de la grémille s'effectue entre $6{ }^{\circ} \mathrm{C}$ et $18{ }^{\circ} \mathrm{C}$ (Keith \& Allardi, 2001; Kolomin, 1977; Ogle, 1998). Le site de l'université du Minnesota révèle que la spermatogenèse a lieu entre $2{ }^{\circ} \mathrm{C}$ et $18^{\circ} \mathrm{C}$ (Butskaya, 1985).

\subsubsection{Le sandre (Stizostedion lucioperca)}

De nombreux articles ont répertorié les tolérances thermiques de l'embryon de sandre (Gillet, 1989; Hokanson, 1977; Küttel et al., 2002 ; 
Lappalainen, 2001 ; Lappalainen et al., 2003). La gamme de températures optimales pour ce stade est comprise entre $12{ }^{\circ} \mathrm{C}$ et $20{ }^{\circ} \mathrm{C}$ (Alabaster \& Lloyd, 1982; Elliott, 1981 in Küttel et al., 2002; Kokurewicz, 1969 in Lappalainen et al., 2003; Muntyan, 1967 in Lappalainen et al., 2003). Une gamme plus large existe avec une limite inférieure entre $3{ }^{\circ} \mathrm{C}$ (Hassler, 1982 in Gillet, 1989) et $8^{\circ} \mathrm{C}$ (Muntyan, 1967 in Lappalainen, 2001) et une limite supérieure à $24{ }^{\circ} \mathrm{C}$ (Elliott, 1981 in Küttel et al., 2002). Deelder \& Willemsen (1964 in Hokanson, 1977) et Muntyan (1967 in Hokanson, 1977) donnent des valeurs d'ILLT et d'IULT comprises entre $9{ }^{\circ} \mathrm{C}$ et $24^{\circ} \mathrm{C}$ qui correspondent à cette gamme large des températures tolérées. Une CT minimum de $7^{\circ} \mathrm{C}$ et une CT max de $25{ }^{\circ} \mathrm{C}$ est également mentionnée par Alabaster \& Lloyd (1982). Cette CT max est choisie en extrême maximum supérieur supporté par le stade. Gillet (1989) observe une durée de 120 degrés jours entre la ponte et l'éclosion, compatible avec la durée de 8 jours à $14,5{ }^{\circ} \mathrm{C}$ citée par Telechea et al. (2008).

Les différents auteurs qui ont étudié le stade larvaire du sandre, donnent des températures optimales comprises entre $14{ }^{\circ} \mathrm{C}$ et $23{ }^{\circ} \mathrm{C}$ (Hokanson, 1977 ; Teletchea et al., 2008 ; site Fishbase), voire $26{ }^{\circ} \mathrm{C}$ (Zhdanova, 1966 in Hokanson, 1977 en Russie et en Finlande). Deux valeurs de températures limites établies en laboratoire ont été également répertoriées concernant le stade larvaire : une IULT à $31^{\circ} \mathrm{C}$ et une ILLT de $6{ }^{\circ} \mathrm{C}$ (Hokanson, 1977).

Le profil thermique du stade juvénile est renseigné par deux auteurs (Hokanson, 1977 ; Horoszewicz, 1973 in Hokanson, 1977). La température optimale s'établit autour de $27,3{ }^{\circ} \mathrm{C}$ et $30{ }^{\circ} \mathrm{C}$ (Hokanson, 1977). Les températures limites supérieures rencontrées en laboratoire se situent entre $34,3^{\circ} \mathrm{C}$ (IULT 1000 minutes) et $37^{\circ} \mathrm{C}$ (CT maximum) pour une acclimatation à 25,6 ${ }^{\circ} \mathrm{C}$ (Horoszewicz, 1973 in Hokanson, 1977). Par souci de cohérence, la température retenue pour représenter l'extrême supérieur maximum est celle de $34{ }^{\circ} \mathrm{C}$.

Pour le stade adulte, les données ont toutes été extraites d'Alabaster \& Lloyd (1982). Deux études de mortalité in situ en Europe de l'Est mentionnent des températures létales entre $31,5^{\circ} \mathrm{C}$ et $37^{\circ} \mathrm{C}$ (Horoszewicz, 1973 en Pologne; Poltavchuk, 1965 en Russie) pour une acclimatation entre $21{ }^{\circ} \mathrm{C}$ et $25,6{ }^{\circ} \mathrm{C}$, avec des températures maximales tolérées de $33,3{ }^{\circ} \mathrm{C}$ en Pologne (Horoszewicz, 1973). Enfin, Willmensen (1977) mesure une CT maximum de $35,4{ }^{\circ} \mathrm{C}$ aux Pays-Bas (acclimatation à $25^{\circ} \mathrm{C}$ ), conservée en extrême maximal supérieur. La gamme de température optimale est choisie identique à celle des juvéniles (optimum entre $27^{\circ} \mathrm{C}$ et $30^{\circ} \mathrm{C}$ ).

Les températures de début et fin de reproduction ont été notées par sept auteurs avec des seuils extrêmes de $3{ }^{\circ} \mathrm{C}$ en Europe de l'Est et $26^{\circ} \mathrm{C}$ en Finlande (Alabaster \& Lloyd, 1982; Raikova-Petrova \& Divkov, 1998; Zhdanova, 1966). Les autres références situent la température limite inférieure autour de $6-8{ }^{\circ} \mathrm{C}$ (Deelder \& Willemsen, 1964 in Gillet, 1989; EIFAC, 1969 in Küttel, et al. 2002; site Fishbase; Fontell et al., 2004 en Finlande; Gillet, 1989; Hokanson, 1977; Mann, 1996; Raikova-Petrova \& Divkov, 1998 en Bulgarie; Teletchea 
et al., 2008) et la température limite supérieure vers $20^{\circ} \mathrm{C}$ (EIFAC, 1969 in Küttel et al., 2002 ; Fontell et al., 2004 en Finlande; Gillet, 1989 ; Hokanson, 1977 ; Mann, 1996).

\subsection{Famille des Esocidae}

\subsubsection{Le brochet (Esox lucius)}

La température optimale pour l'embryon de brochet est comprise entre $8{ }^{\circ} \mathrm{C}$ et $14^{\circ} \mathrm{C}$ (Willemsen, 1959), avec des extrêmes de tolérance à $4{ }^{\circ} \mathrm{C}$ et $23{ }^{\circ} \mathrm{C}$ en laboratoire (Ignatieva \& Kostomarova, 1966 in Saat \& Versaluu, 1996 ; Lillelund, 1966 ; Lindroth, 1946). La température létale minimale se situe à $3{ }^{\circ} \mathrm{C}$ (Hassler, 1982), tandis que Steffens (1976) mentionne la température létale maximale à $21{ }^{\circ} \mathrm{C}$ en Allemagne (valeur plus faible que l'optimum élargi cité). Hassler (1982) mentionne quant à lui une tolérance des embryons de brochet à des variations diurnes de températures de $4{ }^{\circ} \mathrm{C}$ en laboratoire. Les trois données relevées dans la littérature sur la durée du stade embryonnaire (Braum, 1963; Chimits, 1956; Teletchea et al., 2008) suivent la relation établie par Lindroth (1946) :

$$
\mathrm{D}=4+1,26^{19-\mathrm{T}}
$$

$D$ : durée d'incubation et T : température de l'eau en ${ }^{\circ} \mathrm{C}$, dans la gamme $10-14{ }^{\circ} \mathrm{C}$.

Hokanson et al. (1973 in Hokanson, 1977) mesurent une IULT de $28,4{ }^{\circ} \mathrm{C}$ pour la larve et estiment sa température maximale tolérée à $28{ }^{\circ} \mathrm{C}$. L'optimum se situe davantage entre $12{ }^{\circ} \mathrm{C}$ et $21^{\circ} \mathrm{C}$ (Hokanson et al., 1973; Teletchea et al., 2008).
Casselman (1978 in Jobling, 1981) situe entre $19{ }^{\circ} \mathrm{C}$ et $21^{\circ} \mathrm{C}$ la température optimale pour la croissance du juvénile de brochet au Canada. Cvancara et al. (1977) mesurent une LD50 de $30,8{ }^{\circ} \mathrm{C}$ et une IULT à $29,4{ }^{\circ} \mathrm{C}$ dans le Mississippi et Scott (1964 in Hokanson, 1977) une IULT de $33^{\circ} \mathrm{C}$ en laboratoire. La valeur de la LD50 recueillie, équivalente à une $\mathrm{CT}$ max, est choisie comme l'extrême maximum supérieur supporté par le juvénile de brochet.

La température optimale pour le stade adulte s'échelonne entre $10{ }^{\circ} \mathrm{C}$ et $24{ }^{\circ} \mathrm{C}$ (Keith \& Allardi, 2001; McCauley \& Casselman, 1981), avec trois références situant les optimums dans cette gamme (Casselman, 1978 au Canada, in Jobling, 1981; Craig, 1996 in Bruslé \& Quignard, 2001; Hokanson, 1977 in Jobling, 1981). La température extrême maximale est observée à $26{ }^{\circ} \mathrm{C}$ dans l'Ohio aux États-Unis (Bevelhimer et al., 1985) et une gamme élargie à $4-27{ }^{\circ} \mathrm{C}$ sur le Rhône (Ginot et al., 1996). La température létale supérieure est de $34{ }^{\circ} \mathrm{C}$ en laboratoire (Scott, 1964 in Jobling, 1981) et Casselman (1978) observe une température létale inférieure à $0,1^{\circ} \mathrm{C}$ dans l'Ontario (Canada). Une IULT autour de $29{ }^{\circ} \mathrm{C}$ est donnée par Hokanson et al. (1973 in Hokanson, 1977) et Casselman (1978), comparable à la température létale mentionnée par Huet (1962) en milieu naturel. Par souci de cohérence, un maximum supérieur à $31{ }^{\circ} \mathrm{C}$, identique à celui du stade précédent, est retenu par les auteurs.

Enfin, la reproduction a lieu de façon optimale entre $8{ }^{\circ} \mathrm{C}$ (Dubé \& Gravel, 1978) et $15{ }^{\circ} \mathrm{C}$ (Machniak, 1975), avec trois références 
mentionnant des températures optimales dans cet intervalle (Bryan, 1967 aux USA; Dubé \& Gravel, 1978 au Canada; Teletchea et al., 2008). La gamme élargie s'établit entre $6^{\circ} \mathrm{C}$ (Dumont et al., 1980 au Canada; Machniak, 1975) et $20{ }^{\circ} \mathrm{C}$ (Machniak, 1975), deux auteurs citant des températures extrêmes supérieures en laboratoire entre $15{ }^{\circ} \mathrm{C}$ et $20{ }^{\circ} \mathrm{C}$ (Franklin \& Smith, 1963; Hokanson et al., 1973 in Hokanson, 1977). Seuls deux auteurs mentionnent des températures extrêmes inférieures à $4{ }^{\circ} \mathrm{C}$ (Machniak, 1975; Ovidio \& Philippart, 2005 en Belgique).

\subsection{Famille des Centrarchidae}

\subsubsection{La perche soleil (Lepomis gibbosus)}

La durée du stade embryonnaire entre la ponte et l'éclosion de la larve de perche soleil est estimée à 3,1 jours à $22,5^{\circ} \mathrm{C}$ (Teletchea et al., 2008).

La larve possède son optimum à $20,4{ }^{\circ} \mathrm{C}$ pour le site Fishbase, tandis que Teletchea et al. (2008) le situent à une température plus élevée $\left(23,5^{\circ} \mathrm{C}\right)$.

Le juvénile est dans son optimum de température entre $13{ }^{\circ} \mathrm{C}$ et $28{ }^{\circ} \mathrm{C}$ en Grèce (Neophitou \& Giapis, 1994 in Küttel et al., 2002). Une IULT de 30,2 ${ }^{\circ} \mathrm{C}$ est établie par Black (1953 in EPRI, 1981) au Canada. Ferguson (1958 in Coutant, 1977) trouve une température optimale plus élevée en laboratoire $\left(31,5^{\circ} \mathrm{C}\right)$.

Dans la synthèse effectuée par l'EPRI (1981) sur des études réalisées aux États-Unis, l'adulte semble supporter des températures comprises entre $11,9{ }^{\circ} \mathrm{C}$ (Marcy, 1976) et $35^{\circ} \mathrm{C}$
(Neill, 1971; Trembley, 1960). L'optimum de croissance est à $30{ }^{\circ} \mathrm{C}$ pour Brett (1979) et trois données mentionnées par Coutant (1977) sont situées en dessous de $28{ }^{\circ} \mathrm{C}$ (Neill, 1971 in Coutant, 1977; Reutter \& Herdendorf, 1974 in Coutant, 1977) ce qui milite pour une limite supérieure de la gamme optimale autour de cette température. Quatre références (Marcy \& Jacobson, 1976 ; Neill, 1971 ; Trembley, 1960; Wapora, 1977) listées par l'EPRI (1981) donnent des températures maximales plus fortes, davantage comparables aux mesures en laboratoire. Des CT maximum ont été établies en laboratoire : Beitinger et al. (2000) citent des valeurs entre $30,1^{\circ} \mathrm{C}$ et $35,1^{\circ} \mathrm{C}$ (Becker \& Genoway, 1979) selon la température d'acclimatation $\left(10{ }^{\circ} \mathrm{C}\right.$ et $20{ }^{\circ} \mathrm{C}$ respectivement) aux États-Unis. La synthèse de Hagen (1973) mentionne des CT maximums comparables $\left(25,5^{\circ} \mathrm{C}\right.$ à $\left.34,5^{\circ} \mathrm{C}\right)$ observées par Jones (1964). Burns (1973) et Reutter \& Herdendorf (1976) notent quant à eux une CT maximum légèrement supérieure $\left(37,5^{\circ} \mathrm{C}\right)$. La valeur de $35^{\circ} \mathrm{C}$ est retenue par les auteurs comme limite supérieure de la gamme extrême supportée par le stade. Des CT minimum de $1,7{ }^{\circ} \mathrm{C}$ à $12,1^{\circ} \mathrm{C}$ ont également été mesurées pour des températures d'acclimatation de $15{ }^{\circ} \mathrm{C}$ à $30{ }^{\circ} \mathrm{C}$ (Becker et al., 1977 in Beitinger et al., 2000).

Les températures en période de reproduction ne sont pas aussi bien documentées que pour les autres espèces de poissons étudiées. L'extrême supérieur se situe à $25^{\circ} \mathrm{C}$ en Grèce (Neophitou \& Giapis, 1994 in Küttel et al., 2002). La limite inférieure de reproduction pourrait s'établir autour de $13{ }^{\circ} \mathrm{C}$. Trois auteurs mentionnent 
des optimums entre ces deux températures limites (Bruslé \& Quignard, 2001 ; Teletchea et al., 2008; Wiliamson et al., 1993 in Küttel et al., 2002).

\subsection{Famille des Siluridae}

\subsubsection{Le silure (Silurus glanis)}

La durée d'incubation de l'embryon de silure se situe entre 2 et 3 jours à $22-25{ }^{\circ} \mathrm{C}$, soit autour de 55 degrés jours (Bruslé \& Quignard, 2001; Keith \& Allardi, 2001; Lelek \& Penaz, 1963 ; Teletchea et al., 2008).

La température optimale pour le stade larvaire est à $24,5^{\circ} \mathrm{C}$ en laboratoire (Teletchea et al., 2008). La température létale minimale en laboratoire est estimée à $13^{\circ} \mathrm{C}$ (Mihalik, 1982).

La plage des températures optimales pour l'adulte de silure est compris entre $12{ }^{\circ} \mathrm{C}$ et $28{ }^{\circ} \mathrm{C}$ (Abdullayev et al., 1978 ; Berg, 1964 ; Hilge, 1985), mais il peut tolérer une plus large plage de température, de $3^{\circ} \mathrm{C}$ à $30^{\circ} \mathrm{C}$ (Schlumberger et al., 2001). Ces températures extrêmes plus basses que $12{ }^{\circ} \mathrm{C}$ sont confirmées par trois auteurs (David, 2006; Mihalik, 1982; Omarov \& Popova, 1985). Les seules mesures concernant des IULT sont mentionnées dans la synthèse de Vallod (1987). Ces IULT sont comprises entre $30,2^{\circ} \mathrm{C}$ pour une acclimatation à $10^{\circ} \mathrm{C}$ (température létale à $30,5^{\circ} \mathrm{C}$ ) et $33^{\circ} \mathrm{C}$ pour une acclimatation à $20^{\circ} \mathrm{C}$ (température létale à $34,4{ }^{\circ} \mathrm{C}$ ) (Toth et al., 1982 in Vallod, 1987). La valeur intermédiaire de $32{ }^{\circ} \mathrm{C}$ est choisie par les auteurs comme limite supérieure maximale de la gamme élargie des températures supportées.

La reproduction enfin se déroule de façon optimale entre $20^{\circ} \mathrm{C}$ (Bruslé
\& Quignard, 2001; Horvath, 1977) et $25{ }^{\circ} \mathrm{C}$ (Bruslé \& Quignard, 2001; Woynarovich \& Horvath, 1981), deux auteurs mentionnant des optimums en laboratoire dans cette plage de température (Horvart \& Lucowicz, 1982 in Vallod, 1987; Teletchea et al., 2008). Des températures tolérées plus basses sont également reportées au minimum à $17^{\circ} \mathrm{C}$ (Lelek, 1987; Mihalik, 1982 en laboratoire; Mohr, 1957).

\subsection{Synthèse des gammes de tolérance}

Les gammes des températures optimales ainsi que la limite supérieure de la gamme de résistance choisies pour chacun des stades des espèces étudiées sont résumées dans le tableau III (voir paragraphe 2.4 pour la méthodologie appliquée pour établir ce choix). L'ensemble des références sur lesquelles nos choix se sont appuyés est donné en annexe 1. Les températures létales associées sont trop hétérogènes pour permettre leur interprétation.

\section{DISCUSSION}

Nous avons synthétisé les tolérances thermiques des espèces à partir de sources de données présentant une grande disparité géographique et temporelle et des conditions de relevés mixtes, provenant soit d'observations de terrain, soit d'expériences contrôlées en laboratoire. En donnant un accès au tableau détaillant les références utilisées (annexe 1), nous encourageons les lecteurs à enrichir cette base dans le futur. Nous ménageons surtout la possibilité de relativiser l'interprétation des valeurs que nous proposons. 
Tableau III. Gamme des températures optimales et limite supérieure de la gamme de résistance pour chaque espèce.

Table III. Optimum temperature range and upper limit of the resistance temperature range for each species.

\begin{tabular}{|c|c|c|c|c|c|c|c|c|c|c|c|c|c|c|}
\hline & \multicolumn{3}{|c|}{ Embryon } & \multicolumn{3}{|c|}{ Larve } & \multicolumn{3}{|c|}{ Juvénile } & \multicolumn{3}{|c|}{ Adulte } & \multicolumn{2}{|c|}{ Reproduction } \\
\hline & $\begin{array}{l}\text { Opt. } \\
\text { min }\end{array}$ & $\begin{array}{l}\text { Opt. } \\
\max \end{array}$ & $\overline{M A X}$ & $\begin{array}{l}\text { Opt } \\
\text { min }\end{array}$ & $\begin{array}{l}\text { Opt. } \\
\text { max }\end{array}$ & MAX & $\begin{array}{l}\text { Opt. } \\
\text { min }\end{array}$ & $\begin{array}{l}\text { Opt. } \\
\text { max }\end{array}$ & MAX & $\begin{array}{l}\text { Opt. } \\
\text { min }\end{array}$ & $\begin{array}{l}\text { Opt. } \\
\text { max }\end{array}$ & MAX & $\begin{array}{l}\text { Opt. } \\
\text { min }\end{array}$ & MAX \\
\hline BLN & & & & & & & 13 & 15 & & 10 & 18 & 27 & 12 & 15 \\
\hline TOX & & & & 14 & 18 & & & & & 16 & 25 & & 9 & 14 \\
\hline НОТ & 10 & 17 & 20 & 15 & 25 & 28 & 15 & 25 & & 15 & 24 & & 8 & 14 \\
\hline GOU & 16 & 20 & & & & & 7 & 27 & & 7 & 30 & 36 & 12 & 17 \\
\hline CHE & 16 & 24 & 30 & 14 & 25 & & 14 & 25 & 30 & 14 & 24 & 30 & 15 & 20 \\
\hline VAN & 7 & 15 & 25 & 16 & 25 & & 12 & 25 & & 10 & 25 & 32 & 7 & 12 \\
\hline SPI & & & & 19 & 24 & & 12 & 24 & & 12 & 24 & 27 & 14 & 21 \\
\hline BAF & 16 & 21 & & 15 & 19 & 24 & 13 & 24 & 32 & 10 & 24 & 32 & 13 & 20 \\
\hline BOU & & & & & & & & & & 12 & 30 & 37 & 15 & 21 \\
\hline GAR & 12 & 24 & 26 & & & & 7 & 21 & 30 & 12 & 25 & 31 & 10 & 18 \\
\hline ABL & 21 & 27 & 31 & & & & & & & 20 & 30 & 35 & 15 & 28 \\
\hline BRE & 12 & 23 & 28 & & & & 14 & 28 & 34 & 10 & 26 & 35 & 12 & 20 \\
\hline BRB & & & & & & & & & & 16 & 25 & & 15 & 25 \\
\hline PER & 12 & 18 & 21 & 12 & 25 & 34 & 10 & 25 & 32 & 16 & 27 & 33 & 8 & 15 \\
\hline GRE & 9 & 21 & 24 & 25 & 30 & & 7 & 25 & 30 & 15 & 25 & 31 & 6 & 18 \\
\hline SAN & 12 & 20 & 25 & 14 & 23 & 31 & 27 & 30 & 34 & 27 & 30 & 35 & 6 & 20 \\
\hline BRO & 8 & 14 & 23 & 12 & 21 & 28 & 19 & 21 & 31 & 10 & 24 & 31 & 8 & 15 \\
\hline PES & & & & & & & 13 & 28 & 30 & 12 & 30 & 35 & 13 & 25 \\
\hline SIL & & & & & & & & & & 12 & 28 & 32 & 20 & 25 \\
\hline
\end{tabular}

Italique : donnée sans référence associée (issue du stade précédent ou estimée) / italics: data without associated reference (derived from precedent stage or estimated)

Opt. min et max : limites de la gamme optimale / optimal range limits

MAX : limite supérieure de la zone de résistance / upper limit of the resistance range

Quand cela était possible, nous avons privilégié le recours à des données sources. Nous avons également recueilli des données issues de compilations existantes (Alabaster \& Lloyd, 1982 ; Hokanson, 1977; Küttel et al., 2002; Mann, 1996; Teletchea et al., 2008).

Dans le cas des observations in situ, différentes températures tolérées pour une même espèce ou pour un même stade peuvent être mentionnées. Cela peut s'expliquer soit par des différences géographiques de régime thermique (par exemple Kottelat \& Freyhof, 2007 indiquent que la Grémille se reproduit dès $6^{\circ} \mathrm{C}$ au nord de l'Europe et plutôt à partir de $10{ }^{\circ} \mathrm{C}$ au sud), soit par la plasticité des espèces. On sait qu'il existe une variation phénotypique naturelle de tolérance thermique (Schaefer \& Ryan, 2006), mais 
l'amplitude de variabilité et le moment de l'ontogenèse où elle survient sont encore mal connus (Grünbaum et al., 2007). Par exemple, le poisson zèbre (Danio rerio), poisson thermophile à cycle de vie court, montre une plasticité intrinsèque avec une tolérance accrue de l'adulte pour de plus fortes températures si les juvéniles ont euxmêmes été soumis à une forte température (Schaefer \& Ryan, 2006). Mais une autre référence pour Esox masquinongy n'a montré aucune différence de tolérance thermique pour des individus âgés de 2 ans, issus de différentes populations (Wagner et al., 2007). C'est donc une propriété qu'il est difficile de généraliser; on retiendra par contre que les températures réellement viables in situ sont systématiquement plus faibles de l'ordre de $1{ }^{\circ} \mathrm{C}$ à $4{ }^{\circ} \mathrm{C}$ par rapport aux températures létales (Eaton et al., 1995; Magnuson et al., 1979), c'est pourquoi nous conseillons de privilégier comme métrique repère le maximum de la tolérance thermique ( $7^{e}$ colonne du tableau annexe 1).

À ces considérations sur les variations individuelles ou populationnelles, il faut ajouter d'autres connaissances pour bien interpréter les valeurs de tolérance thermique :

- les poissons sont extrêmement sensibles aux températures du milieu ambiant; ils peuvent détecter de très faibles écarts de température de l'ordre de $0,001^{\circ} \mathrm{C}$ (Brown, 2003);

- ils ont tendance à sélectionner des habitats thermiques qui maximisent leur taux de croissance (Magnuson et al., 1979) ;
- la température létale peut être augmentée suite à des périodes d'acclimatation progressives, dans la limite de $3{ }^{\circ} \mathrm{C}$ maximum pour les salmonidés (ex. Saumon atlantique, Garcia de Leaniz et al., 2007). Mais il faut garder à l'esprit que les temps d'acclimatation sont longs, de l'ordre de 20 jours pour les ectothermes (Lutterschmidt \& Hutchison, 1998);

- aux plus fortes températures tolérées, il faut tenir compte des risques potentiels de raréfaction de nourriture et de sensibilité accrue à la toxicité par rapport aux xénobiotiques (Heugens et al., 2001);

- des poissons adultes soumis à des amplitudes thermiques journalières fortes dans la zone de leur température maximale tolérée consomment une énergie supplémentaire soustraite aux fonctions de croissance ou de production de gamètes (Schaefer \& Ryan, 2006 : poisson zèbre, soumis à un régime thermique de $28 \pm 6{ }^{\circ} \mathrm{C}$ ) ;

- au stade larvaire, les poissons sont plus vulnérables aux stress thermiques, car ils ont une plus faible capacité que les adultes à réguler la fluidité de leurs membranes, d'acclimater leur taux métabolique ou de se thermoréguler de façon comportementale (Brett, 1970 ; Rombough, 1997);

- à l'échelle journalière, on doit considérer non seulement la température maximale tolérée, mais aussi sa durée d'imposition, la température de rémission nocturne, et sa durée qui doit être suffisante pour que l'organisme répare ses protéines de stress. À l'échelle de plusieurs jours, il ne faut pas sous 
estimer l'influence d'expositions cumulées dans le temps, à des températures proches des températures maximales tolérées;

- à une échelle locale (quelques mètres à quelques centaines de mètres), il existe des contrastes de température au sein des cours d'eau et les poissons sont capables de rechercher des refuges thermiques, dans les affluents, dans les annexes fluviales, ou dans des afférences phréatiques plus fraîches (Torgersen et al., 2001). Les salmonidés utilisent très efficacement ces contrastes (Torgersen et al., 1999); le comportement d'autres familles est moins documenté : une étude par radiopistage de Cooke et al. (2004) sur le black bass à petite bouche (Micropterus dolomieu), a montré que cette espèce évitait activement les zones les plus chaudes d'un rejet industriel, et qu'elle ne les fréquentait plus jamais une fois qu'elle les avait " expérimentées».

La gamme des températures optimales et la gamme de résistance retenues par les auteurs (Tab. III) sont représentées figure 2 pour les juvéniles et figure 3 pour les adultes. De manière générale, le stade juvénile est nettement moins bien renseigné que le stade adulte. L'extrême supérieur de la gamme de résistance est également une donnée non exhaustive.

Trois groupes d'espèces se dégagent de ces figures, selon leurs exigences thermiques :

- le blageon a un comportement très distinct des autres espèces, avec une croissance à température faible (optimum maximal à $15{ }^{\circ} \mathrm{C}$ pour le juvénile et à $18{ }^{\circ} \mathrm{C}$ pour

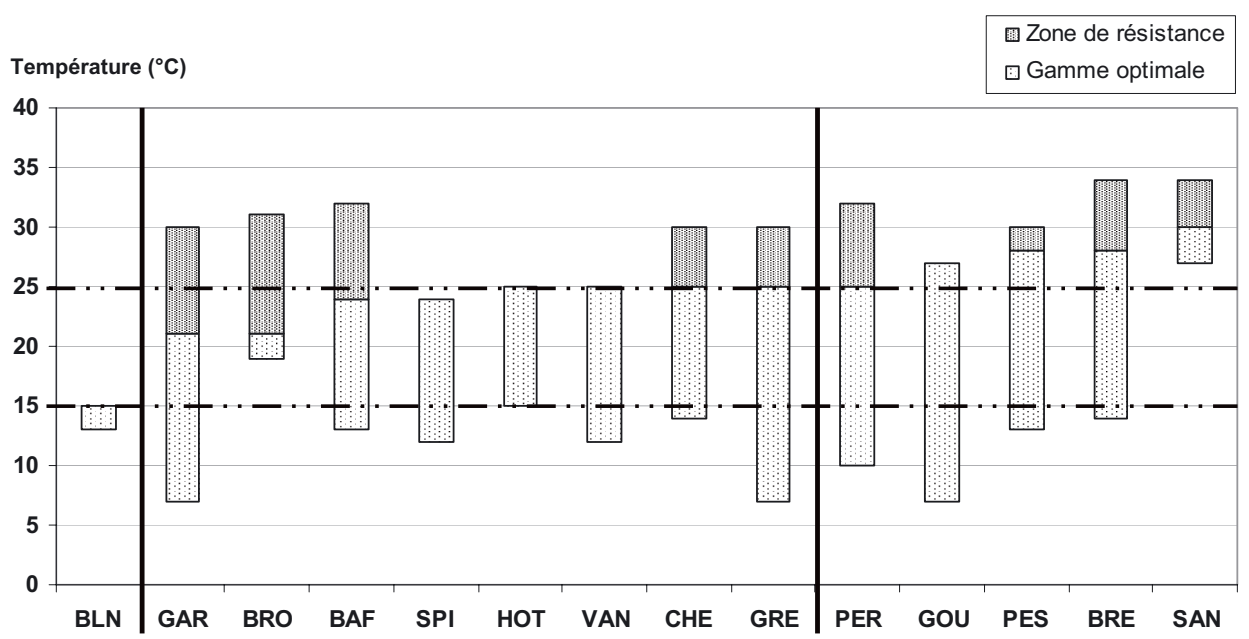

Fig. 2. Représentation de la gamme des températures optimales et de la gamme de résistance à la température pour le juvénile de chacune des espèces étudiées.

Fig. 2. Optimum temperature range and resistance temperature range for juvenile of each studied species. 


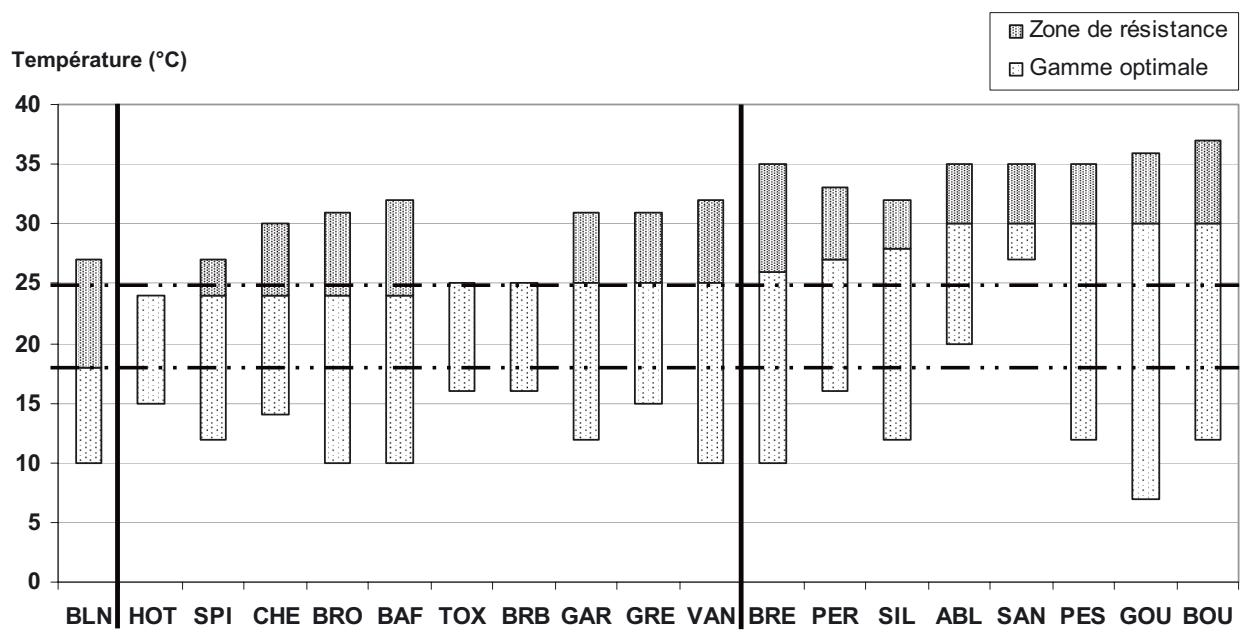

Fig. 3. Représentation de la gamme des températures optimales et de la gamme de résistance à la température pour l'adulte de chacune des espèces étudiées.

Fig. 3. Optimum temperature range and resistance temperature range for adult of each studied species.

l'adulte). II se situe dans la queue de distribution des espèces sténothermes d'eau froide, résultat conforme à la position de l'espèce dans la zonation théorique de Verneaux (1981);

- un groupe que l'on peut qualifier de mésotherme avec le hotu, le spirlin, le chevesne, le brochet, le barbeau fluviatile, le toxostome, la brème bordelière, le gardon, la grémille et la vandoise, qui grandissent à des températures tempérées : optimum maximal autour de 20$25{ }^{\circ} \mathrm{C}$ pour le juvénile et de $25^{\circ} \mathrm{C}$ pour l'adulte; maximum toléré légèrement au-delà de $30^{\circ} \mathrm{C}$;

- un dernier groupe nettement plus tolérant aux températures chaudes, constitué de la brème commune, de la perche fluviatile, du silure, de l'ablette, du sandre, de la perche soleil, du goujon, de la bouvière.
Les adultes sont ainsi dans leur optimum de température jusqu'à 25-30 ${ }^{\circ} \mathrm{C}$ et les maxima tolérés peuvent atteindre $37^{\circ} \mathrm{C}$ (et toujours supérieurs à $32{ }^{\circ} \mathrm{C}$ chez l'adulte).

La figure 4 présente les optimums de température pour la reproduction de ces espèces.

Selon la gamme optimale de reproduction, les 19 espèces étudiées se distinguent en trois groupes :

- un groupe pour lequel la reproduction peut avoir lieu en dessous de $10^{\circ} \mathrm{C}$ : la grémille, le sandre, la vandoise, le hotu, la perche fluviatile, le brochet et le toxostome;

- un groupe intermédiaire qui peut se reproduire entre $10^{\circ} \mathrm{C}$ et $15^{\circ} \mathrm{C}$ : le gardon, le blageon, le goujon, la brème commune, le barbeau 


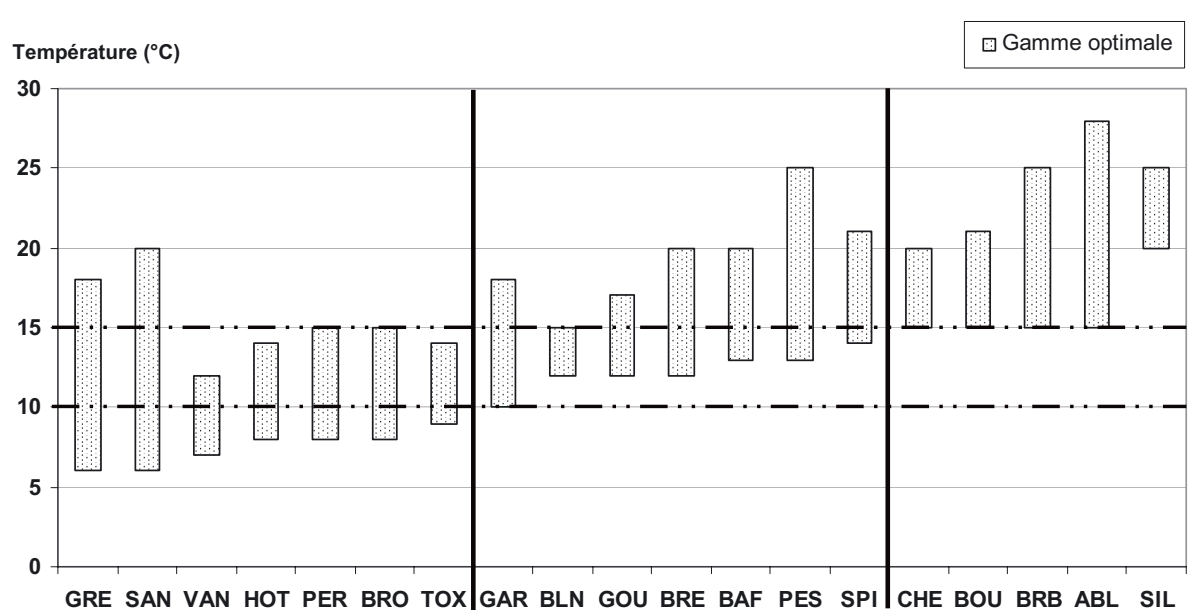

Fig. 4. Représentation de la gamme de température optimale pour la reproduction de chacune des espèces étudiées.

Fig. 4. Optimal temperature range for the reproduction of each studied species.

fluviatile, la perche soleil et le spirlin;

- un groupe dont les espèces se reproduisent à des températures plus chaudes que $15^{\circ} \mathrm{C}$ : le chevesne, la bouvière, la brème bordelière, l'ablette et le silure.

Selon les régimes actuels et futurs, notamment printaniers, de plus en plus précoces dans l'atteinte de températures favorables à la reproduction (GIEC, 2007), certaines espèces résisteront d'autant mieux que l'amplitude de leur tolérance thermique pour la reproduction est large. Parmi elles, la grémille et le sandre ont une large tolérance thermique, à l'inverse de la vandoise, du toxostome ou du blageon. Or les espèces qui présentent les plus fortes amplitudes de tolérance pour la reproduction appartiennent souvent au groupe le plus tolérant pour les stades juvéniles et adultes et sont également souvent dotées d'une stratégie de ponte multiple. Ces espèces seront donc vraisemblablement favorisées lors d'année chaude, ce qui est déjà observé sur le Rhône à Bugey (Daufresne \& Boët, 2007).

Toute typologie comporte une part d'arbitraire et notre classification n'y échappe pas. Nous avons essayé de rendre compte du jeu de données relatif aux 19 espèces analysées, en dégageant 3 seuils pour les métriques d'optimum thermique maximal adulte, $18^{\circ} \mathrm{C}, 25^{\circ} \mathrm{C}$ et $30^{\circ} \mathrm{C}$; n'ayant pas pris en compte dans notre synthèse les salmonidés et d'autres espèces de la zone apicale des cours d'eau, il n'est pas surprenant de ne retrouver que le blageon dans le premier groupe des sténothermes d'eau froide pour lesquels Raat (1988) place la borne supérieure à $19{ }^{\circ} \mathrm{C}$ et l'optimum à $15^{\circ} \mathrm{C}$ et Hokanson (1977) à $20{ }^{\circ} \mathrm{C}$. Nous distinguons assez nettement un groupe de mésothermes, dans lequel se situent majoritairement 
les cyprinidés rhéophiles et un groupe d'espèces plus tolérantes à des températures plus chaudes, avec surtout des cyprinidés plus lénitophiles. Nous apportons des nuances par rapport à Raat (1988), dont les limites pour les mêmes groupes étaient plus resserrées, respectivement $\left(19-21{ }^{\circ} \mathrm{C}\right.$, optimum de croissance $19{ }^{\circ} \mathrm{C}$, et $>21^{\circ} \mathrm{C}$, optimum de croissance, $27{ }^{\circ} \mathrm{C}$ ) ou Hokanson (1977), $\left(<28^{\circ} \mathrm{C}\right.$ et $\left.>28^{\circ} \mathrm{C}\right)$. Concernant certaines espèces, nous classons différemment de Daufresne \& Boët (2007) la brème bordelière (groupe mésotherme selon cette synthèse et non groupe chaud), la perche soleil et la perche (groupe chaud selon cette synthèse et non groupe froid).

Les groupes de tolérance thermique dégagés proposent déjà des valeurs indicatives qui devraient être précieuses pour situer les probabilités de modification des communautés de poissons dans des plages thermiques données. II reste cependant indispensable de porter le raisonnement à la fois sur le régime thermique (intensité, durée, fréquence et saisonnalité des températures) mais également sur les différents stades de vie des espèces.

Nous conseillons d'une part de recourir préférentiellement aux données spécifiques pour effectuer des analyses sur la mise en évidence de tendances rétrospectives, et d'autre part de bien raisonner en analysant plutôt des régimes couplés entre biologie (différents stades des cycles de vie) et température. Ceci sera d'autant plus vrai lorsque l'on tentera d'effectuer des prévisions tendancielles répondant à des scénarios de changements globaux, où il faudra tenir compte non seulement des évolutions saisonnières de température, mais aussi du régime hydrologique modifié (Graham \& Harrod, 2009).

\section{CONCLUSION ET PERSPECTIVES}

La température est une des variables clés du fonctionnement des hydrosystèmes : elle est sujette à des changements locaux en aval des sites urbanisés, des sites industriels ou des espaces ruraux où les corridors boisés pouvant jouer un rôle de tampon thermique ont été fortement réduits; des évolutions plus globales d'origine climatique ont aussi été enregistrées dans les dernières décades et sont également possibles dans le futur selon divers scénarios de changement climatique. Les écologues sont donc naturellement saisis de la question de la sensibilité des milieux à ces changements et de la tolérance des espèces aquatiques. Nous apportons par cette synthèse relative aux poissons des éléments de connaissance jusqu'alors disparates, et parfois peu accessibles quand il s'agit de littérature ancienne. Dans les diagnostics ou les pronostics de sensibilité aux changements thermiques, il est important que les raisonnements soient partageables et reposent sur une base de connaissance commune. Cette synthèse permet ainsi d'identifier des espèces capables de supporter une élévation de température plus facilement que d'autres. Au-delà de ces résultats, il reste néanmoins important de garder à l'esprit que d'autres facteurs interviennent dans la capacité des espèces à résister à une élévation de température. 
Cette synthèse met aussi en évidence les connaissances manquantes pour certains stades ou certaines espèces (en particulier blageon, toxostome, bouvière et brème bordelière) ; cela pourrait guider dans le futur de nouvelles investigations qu'elles soient de nature écologique, par analyse de données de terrain couplées entre températures et présence de poissons ou plus physiologiques en laboratoire sur les limites thermiques tolérées.

Ces résultats pourraient aussi être précieux pour mieux comprendre la répartition géographique actuelle des poissons et interpréter les changements temporels des communautés pour les sites bénéficiant de chroniques suffisamment documentées.

\section{REMERCIEMENTS}

Des experts européens du domaine on été sollicités afin de compléter et de valider le travail de synthèse réalisé. Les auteurs remercient pour leur participation à ce document de synthèse et pour les références fournies : Christian Gillet (INRA, France : brochet, perche fluviatile et gardon), Rodolphe Gozlan (université de Bournemouth, Angleterre : toxostome), Michel Khalanski (comité hydroécologie), Krzysztof Kupren (université de Warmiǹsko-Mazurski, Pologne : vandoise et chevesne), Alain Nelva (université de Savoie, France : hotu), Nicolas Poulet (Onema, France : sandre) et Dominique Vallod (ISARA, France : silure). Les auteurs remercient également pour les considérations générales et les pistes bibliographiques apportées : Jérôme Belliard (Onema, France), Georges
Carrel (Cemagref, France), Cordon Copp (université de Bournemouth, Angleterre), Pascal Fontaine et Fabrice Teletchea (université de Nancy, France) et Martin Reichard (Académie des Sciences, République Tchèque). Enfin, un grand merci également à Federica Oraison pour l'aide précieuse apportée dans la recherche documentaire et une première relecture.

\section{RÉFÉRENCES BIBLIOGRAPHIQUES}

Abdullayev M.A., Khakberdiev B. \& Urchinov D., 1978. Biology of the catfish Silurus glanis from some lakes in the lower Zarafshan River and in the Khorezm district. J. Ichtyol. 17 (3) : 487-491.

Alabaster J.S., 1964. The effect of heated effluents on fish. In International conference on water pollution research N.Y. Pergamon press Ed., pp. 261-292.

Alabaster J.S. \& Downing A.L., 1966. A field and laboratory investigation of the effect of heated effluents on fish. Min. Agric. Fish. Food (Gt. Brit.) Ser. : 1-42.

Alabaster J.S. \& Lloyd R., 1982. Water temperature. In Water quality criteria for freshwater fish. J.S. Alabaster and R. Lloyd Eds., pp. 47-102.

Allan J.D., 1995. Stream ecology : structure and function of running waters. Chapman \& Hall. London, p. 388.

Armour C., 1991. Guidance for evaluating and recommending temperature regimes to protect fish. U.S. Wildlife Service, Washington, p. 13.

Banarescu P., 1964. Fauna republicii populare Romine, Pisce-Osteichthyes. Bucuresti, p. 962.

Banarescu P.M., Bogutskaya N.G., Movchan Y.V.' \& Smirnov A.l., 2003a. Barbus barbus (Linnaeus, 1758). In The freshwater fishes of Europe. Petru Banarescu and Nina G. Bogutskaya Ed., pp. 43-90. 
Banarescu P.M., Soric V.M. \& Economidis P.S., 2003b. Gobio gobio (Linnaeus, 1758). In The freshwater fishes of Europe. Petru Banarescu and Nina G. Bogutskaya Ed., pp. 81-126.

Baras E., 1995. Thermal related variations of seasonal and daily spawning periodicity in Barbus barbus. J. Fish Biol. 46 (5) : 915-917.

Baras E. \& Philippart J.C., 1999. Adaptive and evolutionary significance of a reproductive thermal threshold in Barbus barbus. J. Fish Biol. 55 : 354-375.

Barbier B. \& Pascal M., 1976. Résistance du gardon (Rutilus rutilus L.) à des variations brusques de température. Cahiers du laboratoire d'hydrobiologie de Montereau 3 : 25-34.

Bartholow J., 2000. Estimating cumulative effects of clearcutting on stream temperatures. Rivers 7 (4) : 284-297.

Barus V. \& Prokes M., 1993. Fecundity of the bleak (Alburnus alburnus) in the Vestonice reservoir. Folia Zool. 42 (3) : 281-288.

Bastl I., 1969. Spawning of pike-perch (Stizostedion lucioperca Linneaeus, 1758) in bottom nests in conditions of the Orava Reservoir (Northern Slovakia). Pr. Laboratoria Rybarstva a Hydrobiol. 159-184.

Becker C.D. \& Genoway R.G., 1979. Evaluation of the critical thermal maximum for determining thermal tolerance of freshwater fish. Environ. Biol. Fish. $4: 245-256$.

Becker C.D., Genoway R.G. \& Schneider M.J., 1977. Comparative cold resistance of three Columbia river organisms. Trans. Amer. Fish. Soc. 106 : 178-184.

Beitinger T.L. \& Bennett W.A., 2000. Quantification of the role of acclimation temperature in temperature tolerance of fishes. Environ. Biol. Fish. 58 (3) : 277-288.

Beitinger T.L., Bennett W.A. \& McCauley R.W., 2000. Temperature tolerances of North American freshwater fishes exposed to dynamic changes in temperature. Environ. Biol. Fish. 58 (3) : 237-275.
Berg N., 1949. Ryby presnykh vod SSSR $i$ sopredelnykh stran (Freshawater fishes of the USSR and of adjacent regions). Izdat. AN SSSR, Moska. Leningrad, p. 925.

Berg N., 1964. Freshwater fishes of the U.S.S.R. and adjacent countries, p. $470-474$.

Bernet B., 1960. Recherches biologiques sur les populations de Gobio gobio (Linné 1758) de la Nivelle (fleuve côtier du Pays Basque). Annales de la Station Centrale d'Hydrobiologie Appliquée 8 : 127-180.

Bevelhimer, Stein R.A. \& Carline R.F., 1985. Assessing significance of physiological differences among three esocids with a bioenergetics model. Can. J. Fisheries Aquat. Sci. 42 (1) : 57-69.

Black E.C., 1953. Upper lethal temperatures of some British Columbia freshwater fishes. J. Fish. Res. Board Can. $10: 196-210$.

Bless R., 1996. Reproduction and habitat preference of the threatened spirlin (Alburnoides bipunctatus Bloch) and soufie (Leuciscus souffia Risso) under laboratory conditions (Teleostei : Cyprinidae). In Conservation of endangered freshwater fish in Europe. A. Kirchhofer and D. Hefti Eds., pp. 249-258.

Bliss C.I., 1937. The calculation of timemortality curve. Ann. Appl. Biol. 24 : 815-852.

Bodareu M.N. \& Karlov V.N., 1984. The barbel in the Dniester Basin. Schtiintsa, Kishiniov, p. 139.

Bohl E., Herrmann M., Ott B., Seitz B. \& Heise J., 2004. Untersuchungen zur Fortpflanzungsbiologie, Entwicklung und zu den Lebensräumen von Schneider (Alburnoides bipunctatus BLOCH 1782) und Strömer (Leuciscus souffia agassizi VALENCIENNES 1844) - Abschlussbericht über die Untersuchungen 2002-2004. Bayerisches Landesamt für Wasserwirtschaft, Abteilung Gewässerökologische Forschung, Referat Fischökologie, p. 96.

Bourgeois M., 1963. La vandoise. La Pêche et les Poissons $219: 39$. 
Bourgeois M., 1964. Le goujon. La Pêche et les Poissons 229 : 45.

Braum E., 1963. Die ersten Beutefanghandlungen junger Blaufelchen (Coregonus wartmanni Bloch) und Hechte (Esox lucius L.). Z. Tierpsychologie 20 (3) : 257-266.

Breitenstein M. \& Kirchhofer A., 1999. Biologie, menaces et protection du spirlin (Alburnoides bipunctatus) en Suisse. Office fédéral de l'Environnement, des forêts et du paysage, p. 46.

Brett J.R., 1970. Environmental factors, part I. Temperature. In Marine Ecology. O. Kline Ed., pp. 515-560.

Brett J.R., 1979. Environmental factors and growth. In Fish Physiology, Vol. 8. W.S. Hoar, D.J. Randall and J.R. Brett Eds., pp. 599-675.

Brett J.R. \& Higgs E., 1970. Effect of temperature on the rate of gastric digestion in fingerling sockeye salmon, Oncorhynchus nerka. J. Fish. Res. Board Can. 27 : 1767-1779.

Brown B.R., 2003. Sensing temperature without ion channels. Nature 421: 495.

Brown J.H., Gillooly J.F., Allen A.P., Savage V.M. \& West G.B., 2004. Toward a metabolic theory of ecology. Ecology 85 (7) : 1771-1789.

Brunet R. \& Hoestlandt H., 1972. Recherches biologiques et pisciculture expérimentale du goujon (Gobio gobio L.). Bull. Fr. Pêche Piscic. 246 : 5-32.

Bruslé J. \& Quignard J.P., 2001. Biologie des poissons d'eau douce européens. Lavoisier. Paris, p. 625.

Bryan J.E., 1967. Northern pike production in Phalen Pond, Minnesota. J. Minn. Acad. Sci. 34 (2) : 101-109.

Brylinska M. \& Zbikowska L., 1997. Ecology, growth and fecundity of a reproducing population of silver bream, Blicca bjoerkna, from a lowland river reservoir. Folia Zool. 46 (4) : 353-366.

Burns J.R., 1973. Seasonal changes in an environmental control of gonadal development, respiration, and temperature tolerance limits of the pumpkinseed,
Lepomis gibbosus (pisces: centrarchidae). In University of Massachusetts.

Burton T.M. \& Likens G.E., 1973. Effect of strip-cutting on stream temperatures in Hubbard Brook Experimental Forest, New Hampshire. BioScience 23 : 433-435.

Butskaya N.A., 1985. Analysis of the productivity of spermatogenesis in different periods of the sexual cycle of ruffe, Gymnocephalus cernuus. J. Ichthyol. $25: 44-51$.

Casselman J.M., 1978. Effects of environmental factors on growth, survival, activity, and exploitation of Northern Pike. Am. Fish. Soc. Spec. Publ. 11 : 114-128.

Chappaz R., Brun G. \& Olivari G., 1987. Mise en évidence de différences de régime alimentaire dans une population d'ablettes Alburnus alburnus (L.) dans le lac de Sainte-Croix. Conséquences sur la croissance et la fécondité. Ann. Limnol. 23 (3) : 245-252.

Chappaz R., Brun G. \& Olivari G., 1989. Données nouvelles sur la biologie et l'écologie d'un poisson Cyprinidé peu étudié Chondrostoma toxostoma (Vallot 1936). Comparaison avec Chondrostoma nasus (L., 1766). CR Acad. Sci. Paris 309 (3) : 181-186.

Chimits P., 1956. Le brochet. Bull. Fr. Pêche Piscic. 180 : 81-96.

Cocking A.W., 1959. The effects of high temperatures on roach (Rutilus rutilus) - I. The effects of constant high temperatures. J. Exp. Biol. 36 (1) : 203-216.

Collette B.B., Ali M.A., Hokanson K.E.F., Nagiec M., Smirnov S.A., Thorpe J.E., Weatherley A.H. \& Willemsen J., 1977. Biology of the percids. J. Fish. Res. Board Can. 34 (10) : 1890-1899.

Cooke S.J., Bunt C.M. \& Schreer J.F., 2004. Understanding Fish Behavior, Distribution, and Survival in Thermal Effluents Using Fixed Telemetry Arrays : A Case Study of Smallmouth Bass in a Discharge Canal During Winter. Environ. Manage. 33 (1) : 140-150.

Coutant C.C., 1975. Temperature selection by fish - A factor in power plant impact 
assessments. In Environmental Effects of Cooling Systems at Nuclear Power Plants, p. 25.

Coutant C.C., 1977. Compilation of Temperature Preference Data. J. Fish. Res. Board Can. 34 : 739-745.

Cowles R.B. \& Bogert C.M., 1944. A preliminary study of the thermal requirements of desert reptiles. Bull. Amer. Mus. Nat. Hist. 83 : 265-296.

Cowx I.G. \& Welcomme R.L., 1998. Rehabilitation of rivers for fish; $A$ study undertaken by the European Inland Fisheries Advisory Commission of FAO, p. 260.

Craig J.F., 1978. A study of the food and feeding of perch, Perca fluviatilis L., in Windermere. Freshwater Biol. 8 (1) : 59-68.

Craig J., 1996. Pike: biology and exploitation. Chapman \& Hall, p. 320.

Cvancara A., Stieber S.F. \& B.A. Cvancara, 1977. Summer temperature tolerance of selected species of Mississippi River acclimated young of the year fishes. Comp. Biochem. Physiol., 56 (1A) : 81-85.

Daufresne M. \& Boët P., 2007. Climate change impacts on structure and diversity of fish communities in rivers. Global Change Biol. 13 : 1-12.

Daufresne M., Lengfellner K. \& Sommer U., 2009. Global warming benefits the small in aquatic ecosystems. Proceedings of the National Academy of Sciences of the United States of America 106 (31) : 12788-12793.

Davey A.J.H., Kelly D.J. \& Biggs B.J.F., 2006. Refuge-use strategies of stream fishes in response to extreme low flows. J. Fish Biol. 69 (4) : 1047-1059.

David J.A., 2006. Water quality and accelerated winter growth of European catfish using an enclosed recirculating system. Water Environ. J. 20 (4) : 233-239.

De Bono A., Peduzzi P., Kluser S. \& Giuliani G., 2004. Impacts of summer 2003. United Nations Environment Programme, $4 \mathrm{p}$.
De Vlaming V.L., 1972. Environmental control of teleost reproductive cycles : a brief review. J. Fish Biol. 4 (1) : 131-140.

Deelder C.L. \& Willemsen J., 1964. Synopsis of biological data on pike-perch Lucioperca lucioperca (Linnaeus) 1758. FAO Fisheries Synopsis, p. 57.

Diamond M., 1985. Some observations of spawning by roach, Rutilus rutilus L., and bream, Abramis brama L., and their implications for management. Aquacult. Fish. Manag. 16 : 359-367.

Dubé J. \& Gravel Y., 1978. Plan pilote d'aménagement intégré des ressources biologiques du territoire de la frayère du ruisseau Saint Jean, comté de Châteauguay, Québec. Montréal, p. 52.

Dumont P., Fortin R. \& Fournier H., 1980. Certain aspects of the reproduction of upper Richelieu and Mississipi Bay northern pike, Esox lucius L. 231-248.

Duyvené de Wit J.J., 1955. Some observations on the European bitterling (Rhodeus amarus). S. Afr. J. Wetenskap 51 : 249-251.

Easton K.W. \& Dolben I.P., 1980. The Induced Spawning and Subsequent Survival and Growth of Roach Rutilus rutilus (L.). Aquac. Res. 11 (2) : 59-66.

Eaton J.G., Mccormick J.H., Stefan H.G. \& Hondzo M., 1995. Extreme value analysis of a fish/temperature field database. Ecol. Eng. 4 (4) : 289-305.

Edsall T.A., Selgeby J.H., DeSorcie T.J. \& III J.R.P.F., 1993. Growth-temperature relation for young-of-the-year ruffe. J. Gt. Lakes Res. 19: 630-633.

EIFAC, 1968. Water quality criteria for european freshwater fish. Report on water temperature and inland fisheries based mainly on Slavonic literature, pp. 6-32.

EIFAC, 1969. Water quality criteria for European freshwater fish. Water temperature and inland fisheries. Water Res. 3 : 645-662.

Elliott J.M., 1981. Some aspects of thermal stress on freshwater teleosts. In Stress and fish. A.D. Pickering Ed., pp. 209-245. 
Elliott J.M., 1994. Quantitative ecology and the brown trout. Oxford University Press. Oxford GBR, p. 286.

EPRI, 1981. Literature review: response of fish to thermal discharges. Electric Power Research Institue.

Fairchild D.J. \& McCormick H.J., 1996. Effects of Temperature on Hatching and Development of Ruffe (Gymnocephalus cernuus). J. Gt. Lakes Res. 22 (1) : 8994.

Ferguson R.G., 1958. The preferred temperature of fish and their midsummer distribution in temperate lakes and streams. J. Fish. Res. Board Can. 15 : 607-624.

Filipi N., 1962. Kontribut mbi njohjen e morfologjise e biologjise skobusit te lumit (Chondrostoma nasus ohridanus) ne liqenin e Pogradecit [Contribution à la connaissance de la morphologie et de la biologie du Chondrostome du fleuve (Ch. nasus ohridanus) dans le lac de Pogradec]. Bul. Puni. Ekon. Peshk. Alban. 1 (2) : 146-168.

Filon V.V., 1972. Increase in the temperature at which roach, perch and rudd survive in warm waters from the Knonakovo hydroelectric power station. Hydrobiol. J. 7 (4) : 69-72.

Fontell E., Lehtonen H. \& Lappalainen J., 2004. Influence of temperature and depth on spawning site selection of pikeperch (Sander lucioperca) in the Helsinki sea area. In Proceedings of Percis III. The Third International Percid Fish Symposium T.P.B.a.J.A.M. (Eds), pp. 103-104.

Franklin D.R. \& Smith L.L., 1963. Early Life History of the Northern Pike, Esox lucius L., with Special Reference to the Factors Influencing the Numerical Strength of Year Classes. Trans. Amer. Fish. Soc. 92 (2) : 91-110.

Fry F.E.J., 1971. The effects of environmental factors on physiology of fish. In Fish Physiology : 1-98.

Fry F.E.J., Brett J.R. \& Clawson G.H., 1942. Lethal limits of temperature for young goldfish. Rev. Can. Biol. 1 : 50-56.

Garcia de Leaniz C., Fleming I.A., Einum S., Verspoor E., Jordan W.C.,
Consuegra S., Aubin-Horth N., Lajus D., Letcher B.H., Youngson A.F., Webb J.H., Vollestad L.A., Villanueva B., Ferguson A. \& Quinn T.P., 2007. A critical review of adaptive genetic variation in Atlantic salmon : Implications for conservation. Biol. Rev. 82 (2) : 173-211.

GIEC, 2007. Résumé à l'intention des décideurs. Cambridge University Press. Cambridge, UK et New York, USA, p. 18.

Gillet C., 1989. Le déroulement de la fraie des principaux poissons lacustres. Hydroecol. Appl. 1 : 117-143.

Gillet C. \& Dubois J.P., 2007. Effect of water temperature and size of females on the timing of spawning of perch Perca fluviatilis L. in Lake Geneva from 1984 to 2003. J. Fish Biol. 70 (4) : 1001-1014.

Gillet C. \& Quétin P., 2006. Effect of temperature changes on the reproductive cycle of roach in Lake Geneva from 1983 to 2001. J. Fish Biol. 69 (2) : 518-534.

Gillooly J.F., Brown J.H., West G.B., Savage V.M. \& Charnov E.L., 2001. Effects of size and temperature on metabolic rate. Science 293 (5538) : 2248-2251.

Ginot V., Souchon Y. \& Roger P., 1996. Impact de l'élévation artificielle de température induite par le fonctionnement du Centre Nucléaire de Production Electrique du Bugey (fleuve Rhône) sur les communautés de poissons. Hydroecol. Appl. 8 (1-2) : 1-33.

Gozlan R.E., 1998. Environmental biology \& morphodynamics of the sofie Chondrostoma toxostoma (Cyprinidae), With emphasis on early development, p. 387.

Gozlan R.E., Copp G.H. \& Tourenq J.N., 1999. Comparison of growth plasticity in the laboratory and field, and implications on the onset of juvenile development in the sofie, Chondrostoma toxostoma. Environ. Biol. Fish. 56 : 67-77.

Graham C.T. \& Harrod, C., 2009. Implications of climate change for the fishes of the British Isles. J. Fish Biol. 74 (6) : 1143-1205. 
Grünbaum T., Cloutier R., Mabee P.M. \& Le François N.R., 2007. Early developmental plasticity and integrative responses in arctic charr (Salvelinus alpinus): effects of water velocity on body size and shape. Journal of Experimental Zoology 308B : 396-408.

Gulidov M.V. \& Popova K.S., 1981. The hatching dynamics and morphological features of larvae on roach, Rutilus rutilus in relation to incubation temperature. J. Ichtyol. 1 : 87-92.

Gyurko S., Szabo S., Andreka F., Mircea M., Dimoftache M. \& Creteanu A., 1957. Reproducera artificiala a cleanului si mrenei la Manastur-Cluj (Artificial reproduction of the chub and barlel in the Manastur-Cluj). Bul. Inst. de Cerc. pisc. 16 (2) : 46-52.

Hagen K.H., 1973. Fish behavior related to thermal pollution. Environmental impact of river Mech 11: 1-23.

Hassler T.J., 1982. Effect of temperature on survival of northern pike embryos and yolk sac larvae. Prog. Fish-Cult. 44 (4) : 174-178.

Herzig A. \& Winkler H., 1985. Der Einfluss der Temperatur auf die embryonale Entwicklung der Cypriniden. Oesterreichs Fischerei 38 : 182-196.

Herzig A. \& Winkler H., 1986. The influence of temperature on the embryonic development of three cyprinid fishes, Abramis brama, Chalcalburnus chalcoides mento and Vimba vimba. J. Fish Biol. 28 (2) : 171-181.

Heugens E.H.W., Hendriks A.J., Dekker T., Van Straalen N.M. \& Admiraal W., 2001. A review of the effects of multiple stressors on aquatic organisms and analysis of uncertainty factors of use in risk assessment. Crit. Rev Toxicol. 31 : 247-284.

Hickling R., Roy D.B., Hill J.K., Fox R. \& Thomas C.D., 2006. The distributions of a wide range of taxonomic groups are expanding polewards. Global Change Biol. 12 (3) : 450-455.

Hilge V., 1985. Influence of temperature on the growth of the European catfish (Silurus glanis). Z. Angew Ichthyol. 1 (1) : 27-31.
Hokanson K.E.F., 1977. Temperature requirements of some percids and adaptations to the seasonal temperature cycle. J. Fish. Res. Board Can. 34 : 1524-1550.

Hokanson K.E.F. \& Kleiner C.F., 1974. Effects of constant and rising temperature on survival and developmental rates of embryonic and larval yellow perch, Perca flavescens (Mitchill). In The Early Life History of Fish. J.H.S. Blaxter Ed., pp. 437-448.

Hokanson K.E.F., McCormick J.H. \& Jones B.R., 1973. Temperature requirements for embryos and larvae of the northern pike, Esox lucius (Linnaeus). Trans. Amer. Fish. Soc. 102 : 89-100.

Holcik J., 2003. Rhodeus sericeus (Pallas, 1776). In The freshwater fishes of Europe. P.B.a.N.G. Bogutskaya Ed., pp. 81-126.

Holcik J. \& Hensel, 1971. Ihtyologika prirucika. Obzor, Bratislava, pp. 109-110.

Holcik J. \& Hruska V., 1966. On the spawning substrate of the roach Rutilus rutilus (Linnaeus, 1758) and bream - Abramis brama (Linaeus, 1758) and notes on the ecological characteristic of some european fishes. Vestnik Ceskoslovenske Spolecnosti Zoologicke 30 (1) : 22-29.

Holker H., 2006. Effects of body size and temperature on metabolism of bream compared to sympatric roach. Anim. Biol. 56 (1) : 22-37.

Horoszewicz L., 1971. Lethal temperatures of roach fry (Rutilus rutilus L.) from lakes with normal and artificially elevated temperature. Polskie Archiwum Hydrobiologii 18 (1) : 69-79.

Horoszewicz L., 1972. The influence of parasites, handling of fish and the methods of investigations on the evaluation of their tolerance and thermal resistance. Rocz Nauk Roln 94 : 35-53.

Horoszewicz L., 1973. Lethal and disturbing temperatures in some fish species from lakes with normal and artificially elevated temperature. J. Fish Biol. 5 : 165-181.

Horvart L. \& Lucowicz V.M., 1982. Tables with data of hatchery procedures and 
rearing process of some bred warmwater fishes. Aquacult. Hung. 3 : 212-219.

Horvath L., 1977. Improvement of the method for propagation, larval and post-larval rearing of the Wels (Silurus glanis). Aquaculture 10 (2) : 161-167.

Houston A.H., 1982. Thermal effects upon fishes. National Research Council Canada, p. 200.

Huet M., 1962. Influence du courant sur la distribution des poissons dans les eaux courantes. Rev. Suisse Hydrol. $24: 412-431$.

Hynes H.B.N., 1970. The ecology of running waters. Liverpool University Press. Liverpool GBR, p. 555.

Ignatieva G.M. \& Kostomarova A.A., 1966. The duration of mitotic cycle at the period of synchronous segmentation (T0) and its dependence on temperature in pack embryos. Dokl. Akad. Nauk SSSR 168 (5) : 1221-1224.

Intergovernmental Panel on Climate Change, 2007. Climate change 2007. Synthesis report. A contribution of Working Groups I, II, and III to the Fourth Assessment Report of the Intergovernmental Panel on Climate Change. Cambridge, UK.

Iwama G.K., Thomas P.T., Forsyth R.B. \& Vijayan M.M., 1998. Heat shock protein expression in fish. Rev. Fish Biol. Fisheries 8 (1) : 35-56.

Jobling M., 1981. Temperature tolerance and the final preferendum - rapid methods for the assessment of optimum growth temperatures. J. Fish Biol. 19 (4) : 439-455.

Jones E.J.R., 1964. Thermal pollution: the effect of heated effluents. Butterworth and Co. London, pp. 153-168.

Kamler E., 2002. Ontogeny of yolk-feeding fish : an ecological perspective. Rev. Fish Biol. Fisheries 12 (1) : 79-103.

Kamler E., Keckeis H. \& BauerNemeschkal E., 1998. Temperatureinduced changes of survival, development and yolk partitioning in Chondrostoma nasus. J. Fish Biol. 53 (3) : 658-682.
Kammerer P., 1907. Hybridization of river perch (Perca fluviatilis L.) and ruff (Acerina cernua L.). Arch. Entwicklungsmech. Org. 23 : 511-555.

Karas P., 1987. Food consumption, growth and recruitment in perch (Perca fluviatilis L.).

Karas P., 1990. Seasonal changes in growth and standard metabolic rate of juvenile perch, Perca fluviatilis L. J. Fish Biol. 37 (6) : 913-920.

Karas P., 1996. Basic abiotic conditions for production of perch (Perca fluviatilis L.) young-of-the-year in the Gulf of Bothnia. Ann. Zool. Fennici 33 : 371-381.

Kaushal S.S., Likens G.E., Jaworski N.A., Pace M.L., Sides A.M., Seekell D., Belt K.T., Secor D.H. \& Wingate R.L., 2010. Rising stream and river temperatures in the United States. Front. Ecol. Environ. $8: 461-466$.

Keckeis H., Kamler E., Bauer-Nemeschkal E. \& Schneeweiss K., 2001. Survival, development and food energy partitioning of nase larvae and early juveniles at different temperatures. J. Fish Biol. 59 (1) : 45-61.

Keith P. \& Allardi J., 2001. Atlas des poissons d'eau douce de France. Paris, p. 387.

Kennedy B., 1969. Spawning and early development of the dace Leuciscus leuciscus (L.). J. Fish Biol. 1 : 249-259.

Kennedy M. \& Fitzmaurice P., 1972. Some aspects of the biology of gudgeon Gobio gobio (L.) in Irish waters. J. Fish Biol. 4 : 425-440.

Kinouchi T., 2007. Impact of long-term water and energy consumption in Tokyo on wastewater effluent: implications for the thermal degradation of urban streams. Hydrol. Process. 21 : 12071216.

Kishi D., Murakami M., Nakano S. \& Maekawa K., 2005. Water temperature determines strength of top-down control in a stream food web. Freshwater Biol. 50 (8) : 1315-1322.

Kokurewicz B., 1969. The influence of temperature on the embryonic 
development of the perches : Perca fluviatilis (L.) and Lucioperca lucioperca (L.). Zoolog. Pol. 19 : 47-67.

Kolomin Y.M., 1977. The Nadym River ruffe, Acerina cernua. J. Ichthyol. 17 : 345-349.

Konečná M., Jurajda P. \& Reichard M., 2009. River discharge drives recruitment success of the European bitterling Rhodeus amarus in a regulated river in central Europe. J. Fish Biol. 74 : 16421650.

Kottelat M. \& Freyhof J., 2007. Handbook of European freshwater fishes. Kottelat, Cornol, Switzerland and Freyhof, Berlin, Germany, p. 646.

Kovalev P.J., 1973. Natural reproductive conditions of the pike-perch (Lucioperca lucioperca L.), perch (Perca fluviatilis L.) and ruffe (Acerina cernua L.) of lake IImen. J. Ichthyol. $13: 943-946$.

Kraiem M.M. \& Pattee E., 1980. La tolérance à la température et au déficit en oxygène chez le Barbeau (Barbus barbus L.) et d'autres espèces provenant des zones piscicoles voisines. Arch. Hydrobiol. 88 (2) : 250-261.

Krupka I., 1988. Early development of the barbel [Barbus barbus (Linnaeus, 1758)]. Pr. Ustavu Ryb. Hydrobiol. Bratisl. 6 : 115-138.

Kryzhanovskii S.G., 1949. Ecological and morphological trends in development of the Cyprinoidae and Siluroidae. Trudy Institute Morfology Zhivotn Akademic Nauk 1 (5) : 332.

Kucharczyk D., Luczynski M., Kujawa R., Kaminski R., Ulikowski D. \& Brzuzan P., 1998. Influences of temperature and food on early development of bream (Abramis brama L.). Arch. Hydrobiol. $141: 243-256$.

Kucharczyk D., Targoñska K., Zarski D., Kujawa R. \& Mamcarz A., 2008. A review of the reproduction biotechnology for fish from the genus Leuciscus. Arch. Pol. Fish. 16 (4) : 319-340.

Kupren K., Mamcarz A., Kucharczyk D., Prusiï£¡ska M. \& Krejszeff S., 2008.
Influence of water Temperature on Eggs Incubation Time and Embryonic Development of Fish From Genus Leuciscus. Polish Journal of Natural Science 23 (2) : 461-481.

Küttel S., Peter A. \& Wüest A., 2002. Temperaturpräferenzen und -limiten von fischarten Schweizerischer fliessgewässer, p. 41.

Kwiatkowski M., Zarski D., Kucharczyk D., Kupren K., Jamróz M., Targoñska K., Krejszeff S., Hakuc-Btazowska A., Kujawa R. \& Mamcarz A., 2008. Influence of feeding natural and formulated diets on chosen rheophilic cyprinid larvae. Arch. Pol. Fish. 16 (4) : 383-396.

L'Abbée-Lund J.H. \& Vøllestad L.A., 1985. Homing precision of roach, Rutilus rutilus in lake Arunngen, Norway. Environ. Biol. Fish. 13 (3) : 235-239.

Lam T.J., 1983. Environmental influences on gonadal activity. New York and London : Academic Press, pp. 65-116.

Lapkin V.V., Poddubnyy A.G. \& Svirskiy A.M., 1983. Thermoadaptive properties of fishes from temperate latitudes. J. Ichthyol. 23 (2) : 45-54.

Lappalainen J., 2001. Effects of environmental factors, especially temperature, on the population dynamics of pikeperch (Stizostedion lucioperca (L.)). In Department of Limnology and Environmental Protection 28.

Lappalainen J., Dörner H. \& Wysujack K., 2003. Reproduction biology of pikeperch (Sander lucioperca (L.) - a review. Ecol. Freshw. Fish. 12 (2) : 95-106.

Lelek A., 1987. Threatened fishes of Europe. The freshwater fishes of Europe, p. 343.

Lelek A. \& Penaz M., 1963. Spawning of Chondrostoma nasus (L.) in the Brumovka River. S. Zool. listy. 12 (2) : 121-134.

Lepičová A., Hamáčková J. \& Lepič P., 2002. Rearing of early fry (Leuciscus leuciscus L.) under controlled conditions. Bul. Vurh Vodnany 37 : 16-23. 
Leynaud G., 1967. Les pollutions thermiques - Influence de la température sur la vie aquatique. Bulletin technique d'information $224: 25$.

Leynaud G. \& Allardi J., 1974. Incidences d'un rejet thermique en milieu fluvial sur les mouvements des populations ichtyologiques. Bull. Fr. Piscic. 255 : 41-50.

Lillelund K., 1966. Versuche zur erbrutung der eier vom hecht. Esox lucius in, Abhangigkiet von Temperatur und Licht. Arch. Fishereiwess 17 (2) : 95-113.

Lindroth A., 1946. Zur Biologie der Befruchtung und Entwicklung beim Hecht. Mitt. Anst. Binnenfischerei Drottningholm 24 : 1-173.

Löffler H., 1982. Zur Ökologie des Brachsen (Abramis brama (L.)) im Bodensee. In Universität Tübingen.

Lusk S., 1967. Population dynamics of Chondrostoma nasus (Linnaeus, 1758) in the Rokytna river. Acta Sc. Nat. Brno 1 (12) : 473-522.

Lusk S., 1995. Influence of valley dams on the changes in fish communities inhabiting streams in the Dyje River drainage area. Folia Zool. 44 (1) : 45-56.

Lutterschmidt W.I. \& Hutchison V.H., 1998. The critical thermal maximum : history and critique. Can. J. Zool. 75 : 15611574.

Machniak K., 1975. The effects of hydroelectric development on the biology of northern fishes, III : Walleye, Stizostedion vitreum vitreum: A literature review and bibliography. Canadian Fisheries Marine Service, p. 68.

Magnuson J.J., Crowder L.B. \& Medvick P.A., 1979. Temperature as an ecological resource. Amer. Zool. 19 : 331-343.

Maier K.J., Zeh M., Ortlepp J. \& Zbinden S., 1995. Distribution et reproduction des espèces du genre Chondrostoma en Suisse : le nase (C. nasus), la sofie (C. toxostoma), la savetta (C. soetta). Office fédéral de l'Environnement, des forêts et du paysage, p. 62.

Mamcarz A., Kupren K., Kucharczyk D. \& Kujawa R., 2005. Influence of temperature on, body size and yolk sac vol- umes of three larval rheophilic cyprinids (genus Leuciscus). Europ. Aquacult. Soc., special publication 36 : 310-313.

Mann R.H.K., 1974. Observations on the age, growth, reproduction and food of the dace Leuciscus leuciscus (L.), in two rivers in southern England. J. Fish Biol. 6 : 237-253.

Mann R.H.K., 1996. Environmental requirements of European non-salmonid fish in rivers. Hydrobiologia 323 : 223-235.

Mann R.H.K. \& Bass J.A.B., 1997. The critical water velocities of larval roach (Rutilus rutilus) and dace (Leuciscus leuciscus) and implications for river management. Regul. Rivers: Res. Mgmt. 13 : 295-301.

Marcy B.C.J., 1976. Fishes of the lower Connecticut river and the effects of the Connecticut yankee plant. In The Connecticut river ecological study The impact of a nuclear power plant. D.M.a.L.M. Thorpe Ed., pp. 61-139.

Marcy B.C. \& Jacobson P.M., 1976. Early life history studies of american shad in the lower Connecticut River and effects of the Connecticut yankee plant. Amer. Fish. Soc.

Matthews W.J. \& Maness J.D., 1979. Critical thermal maxima, oxygen tolerance and success of cyprinid fishes in a southwestern river. Am. Midl. Nat. : 374-377.

McCauley R.W. \& Casselman J.M., 1981. The final preferendum as an index of the temperature for optimum growth in fish. In World Symposium on Aquaculture in Heated Effluents and Recirculation Systems B. K. Tiews. Heenemann Verlagsgesellschaft Ed., pp. 81-93.

Middaugh D.P., Davis W.R. \& Yokum R.L., 1975. The response of larval fish, Leiostomus xanthurus, to environmental stress following sublethal cadmium exposure. Contrib. Mar. Sci. 19 : 13-19.

Mihalik J., 1982. Der Wels. Die Neue Brehm-Bücherei, p. 71.

Miller S.W., Wooster D. \& Li J., 2007. Resistance and resilience of macroinvertebrates to irrigation water 
withdrawals. Freshwater Biol. 52 (12) : 2494-2510.

Millet, 1989. Etude morphométrique et scalimétrique des stocks de vandoises du Rhône et de l'Ain - Utilisation dans le marquage biologique, p. 120.

Mills C.A., 1980. Spawning and rearing eggs of the dace. Fish. Manag. 11 (2): 67-72.

Mills C.A., 1981. Egg population dynamics of naturally spawning dace, Leuciscus leuciscus (L.). Environ. Biol. Fish. 6 (2) : 151-158.

Mills C.A., 1991. Reproduction and life history. Chapman \& Hall. London, pp. 483508.

Mohr E., 1957. Der Wels. Die neue Bücherei, p. 44.

Moore R.D., Spittlehouse D.L. \& Story A., 2005. Riparian microclimate and stream temperature response to forest harvesting : A review. J. Am. Water Resour. Assoc. 41 (4) : 813-834.

Mouthon J. \& Daufresne M., 2006. Effects of the 2003 heatwave and climatic warming on mollusc communities of the Saône : a large lowland river and of its two main tributaries (France). Global Change Biol. 12 (3) : 441-449.

Müller R., $1997 . \quad$ Vorlesungsskript Fischkunde der Schweiz 1997/8.

Muntyan S.P., 1967. Effect of constant incubation temperatures on the hatching process and on the morphological characteristics of pike-perch embryos. Trezisy Dokl. Vses. Soveshch. po ekol. i fiziol. ryb. Nauka: 135-140.

Neill W.H., 1971. Distributional ecology and behavioral thermoregulation of fishes in relation to heated effluent from a steam-electric power plant (lake Monona, Wis.).

Neophitou C. \& Giapis A.J., 1994. A study of the biology of pumpkinseed (Lepomis gibbosus (L.)) in Lake Kerkini (Greece). J. Appl. Ichthyol. 10 (2-3) : 123-133.

Noges P. \& Jarvet A., 2005. Climate driven changes in the spawning of roach (Rutilus rutilus (L.)) and bream
(Abramis brama (L.)) in the Estonian part of the Narva River basin. Boreal Environ. Res. 10 : 45-55.

Nzau Matondo B., Ovidio M., Poncin P., Kakesa T.A., Wamuini L.S. \& Philippart J.-C., 2007. Hybridization success of three common European cyprinid species, Rutilus rutilus, Blicca bjoerkna and Abramis brama and larval resistance to stress tests. Fish. Sci. 73 (5) : 1137-1146.

Ogle D.H., 1998. A Synopsis of the Biology and Life History of Ruffe. J. Gt. Lakes Res. 24 (2) : 170-185.

Ojanguren A.F. \& Brana F., 2000. Thermal dependence of swimming endurance in juvenile brown trout. J. Fish Biol. 56 : 1342-1347.

Olden J.D. \& Naiman R.J., 2010. Incorporating thermal regimes into environmental flows assessments : Modifying dam operations to restore freshwater ecosystem integrity. Freshwater Biol. 55 (1) : 86-107.

Omarov O.P. \& Popova O.A., 1985. Feeding behaviour of pike, Esox lucius, and catfish, Silurus glanis, in the Arakum Reservoirs of Dagestan. J. Ichtyol. 25 : 25-36.

Ovidio M. \& Philippart J.C., 2005. Long range seasonal movements of northern pike (Esox lucius L.) in the barbel zone of the River Ourthe (River Meuse basin, Belgium). In Aquatic telemetry : advances and applications. Proceedings of the Fifth Conference on Fish Telemetry held in Europe. M.T.L. G. Spedicato and G. Marmulla Ed., p. 295 .

Papadopol M. \& Cristofor S., 1980. Recherches sur l'écologie de deux populations de spirlin, Alburnoides $B$. bipunctatus (Bloch), des eaux de la Roumanie (Pisces, Cyprinidae). Trav. Mus. Hist. Nat. "Grigore Antipa" XXII : 483-493.

Peczalska A., 1979. Fishery and some problems of the biology of the white bream in the Firth of Szczecin. Prade MIR $17: 145-157$.

Pelz G.R., 1986. Der Schneider. Allg. Fischereizeitung Fischwaid. 
Penaz M., 1973. Embryonic development of the Barb, Barbus barbus (Linnaeus, 1978). S. Zool. listy. 22 (4) : 363-374.

Penaz M., 1974a. Early development of the nase carp, Chondrostoma nasus (Linnaeus, 1758). S. Zool. listy. 23 : 275-288.

Penaz M., 1974b. Influence of water temperature on incubation and hatching in Chondrostoma nasus (Linnaeus, 1758). S. Zool. listy. 23 (1) : 53-59.

Penaz M. \& Sterba O., 1969. Notes to the incubation period, growth and mortality of the chub, Leuciscus cephalus (Linne, 1758), in the early life-history stages. Vestnik Ceskoslovenske Spolecnosti Zoologicke 33 : 56-70.

Penaz M., Lusk S. \& Povz M., 1995. Age and size structure of nase spawning shoals in the artificial spawning canal on the River Sava at Mavcice, Slovenia. Folia Zool. 44 (suppl. 1) : 35-42.

Perche, 1948. Les chondrostomes: le hotu, la soiffe. L'hameçon $27: 11-173$.

Petersen J.H. \& Kitchell J.F., 2001. Climate regimes and water temperature changes in the Columbia River : bioenergetic implications for predators of juvenile salmon. Can. J. Fisheries Aquat. Sci. 58 (9) : 1831-1841.

Philippart J.C., 1980. Démographie du hotu, Chondrostoma nasus (Linné) (Teleostei: Cyprinidae) dans l'Ourthe (bassin de la Meuse, Belgique). Annales de la Société Royale de Zoologie, Belgique 110 (3-4) : 199-219.

Philippart J.C., 1981. Ecologie d'une population de vandoise, Leuciscus leuciscus (L.) dans la rivière Ourthe (bassin de la Meuse, Belgique). Ann. Limnol. 17 (1) : 41-62.

Philippart J.C., Lambert J.M. \& Baras E., 1989. Etude de la conservation, de l'amélioration et de la restauration des habitats utilisables par les poissons de rivière. Vol. 1: 74 pages ; Vol. 72: 87 pages ; Vol. 73 : 86 pages.

Poltavchuk M.A., 1965. Biologia i rozvedenie dneprovskogo sudaka $v$ zamknutvkh vodoemov. Naukowa dumka.
Poncin P., 1996. Reproduction chez nos poissons. Nelles Imprimeries Havaux, p. 80.

Prignon C., Micha J.C. \& Gillet A., 1988. Biological and Environmental Characteristics of Fish Passage at the Tailfer Dam on the Meuse River, Belgium. Trans. Amer. Fish. Soc. : 6984.

Prokes M. \& Penaz M., 1978. The course of spawning, early development and longitudinal growth of the nase carp, chondrostoma nasus, in the Rokytna and Jihlaya rivers. Folia Zool. 27 (3) : 269278.

Quinn T.P. \& Adams D.J., 1996. Environmental changes affecting the migratory timing of american shad and sockeye salmon. Ecology 77 (4) : 1151-1162.

Raat A.J.P., 1988. Synopsis of biological data on the northern pike Esox lucius Linnaeus, 1758. FAO Fish.Synop. (30) Rev.2. Rome : 178.

Raikova-Petrova G. \& Divkov M., 1998. Maturity, spawning and sex ratio of pike perch, Stizostedion luciopevca (L.), in two Bulgarian reservoirs as compared to other European habitats. J. Appl. Ichthyol. 14 (1-2) : 31-35.

Reutter J.M. \& Herdendorf C.E., 1974. Environmental evaluation of a nuclear power plant on Lake Erie. U.S. Fish and Wildlife Service, p. 84.

Reutter J.M. \& Herdendorf C.E., 1976. Thermal discharge from a nuclear power plant: predicted effects on lake Erie fish. Ohio J. Sci. $76:$ 39-45.

Roenko, 1965. White bream of Volgogradskoye dam reservoir. Tr. Saratov. Otd. NII Ozer. Rech. Ryb. Khoz. 8 : 163-170.

Rombough P.J., 1997. The effects of temperature on embryonic and larval development. In Global Warming: Implications for Freshwater and Marine Fish. C.M.M. Wood, D.M., Ed.

Saat T. \& Veersalu A., 1996. The rate of early development in perch Perca fluviatilis L. and ruffe Gymnocephalus cernuus (L.) at different temperatures. Ann. Zool. Fennici 33 : 693-698. 
Schaefer J. \& Ryan A., 2006. Developmental plasticity in the thermal tolerance of zebrafish Danio rerio. J. Fish Biol. 69 (3) : 722-734.

Schiemer F., Keckeis H. \& Kamler E., 2003. The early life history stages of riverine fish : ecophysiological and environmental bottlenecks. Comp. Biochem. Physiol. Pt A 133 (3) : 439-449.

Schiemer F., Keckeis H., Nemeschkal H., Schludermann E., Winkler G. \& Zweimuller I., 2004. Ontogenetic patterns in thermal adaptation of fish vs. long-term temperature trends in large rivers. Int. Congr. 1275 : 209-217.

Schlumberger O., Sagliocco M. \& Proteau J.P., 2001. Biogéographie du Silure glane (Silurus glanis): causes hydrographiques, climatiques et anthropiques. Bull. Fr. Pêche Piscic. 357-360 : 533-547.

Schwarz M., 1996. Verbreitung und Habitatanprüche des Strömers (Leuciscus souffia Risso 1826) in den Fliessgewässern der Schweiz.

Scott D.P., 1964. Thermal resistance of pike (Esox lucius L.), muskellunge ( $E$. masquinongy Mitchill), and their F1 hybrid. J. Fish. Res. Board Can. 21: 1043-1049.

Shiri Harzevili A., De Charleroy D., Auwerx J., Vught I. \& Van Slycken J., 2003. Larval rearing of chub, Leuciscus cephalus (L.), using decapsulated Artemia as direct food. J. Appl. Ichthyol. 19 : 123-125.

Shkorbatov G.L., 1964. Nauch. Dokl. Wysh. Shkoly Biolog. Nauki. 2 : 60-65.

Spillmann C.J., 1961. Poissons d'eau douce. Paul Lechevalier. Paris, p. 303.

Spillmann J., 1962. Sur la systématique de telestes soufia risso. Variation de certains caractères numériques et métrique de l'espèce. Bulletin du Museum National d'Histoire Naturelle 34 (6) : 435-452.

Spurny P., Fiala J. \& Mares J., 2004. Intensive rearing of the nase Chondrostoma nasus (L.) larvae using dry starter feeds and natural diet under controlled conditions. Czech J. Anim. Sci. 49 (10) : 444-449.
Stankovitch S., 1921. Etude sur la morphologie et la nutrition des alevins de poissons cyprinidés. Travaux du laboratoire de pisciculture de l'université de Grenoble, XIIlème année (fascicule unique) : 1-182.

Statova M.P., 1973. Sexual maturity, reproduction and fecundity. In Kuchurganskij liman-okhladitel Moldavsky, pp. 148-149.

Steffens W., 1976. Zeitschrift fur die Binnenfischerei der DDR 23 : 327-343, 360-371.

Swift D.R., 1965. Effect of the temperature on mortality and rate of development of the eggs of the pike (Esox lucius L.) and the perch (Perca fluviatilis L.). Nature $206: 528$.

Talmage S.S., 1977. Thermal effects on aquatic organisms : an annotated bibliography of the 1977 literature. The Ecological Sciences Information Center, p. 179.

Tarkan A.S., Gaygusuz O., Gürsoy C. \& Acipinar H., 2005. Life History Pattern of an Eurasian Cyprinid, Rhodeus amarus, in a Large Drinking-Water System (Ömerli Dam Lake-Istanbul, Turkey). J. Black Sea/Mediterranean Environment 11: 205-224.

Teletchea F., Fostier A., Le Bail P.Y., Jalabert B., Gardeur J.N. \& Fontaine P., 2007. STOREFISH : A new database dedicated to the reproduction of temperate freshwater teleost fishes. Cybium 31 (2) : 227-235.

Teletchea F., Fostier A., Kamler E., Gardeur J.N., Le Bail P.Y., Jalabert B. \& Fontaine P., 2008. Comparative analysis of reproductive traits in 65 freshwater fish species : application to the domestication of new fish species. Rev. Fish Biol. Fisheries : 28.

Teletchea F., Gardeur J.N., Kamler E. \& Fontaine P., 2009. The relationship of ovocyte diameter and incubation temperature to incubation time in temperate freshwater fish species. J. Fish Biol. 74 (3) : 652-668.

Thorpe J.E., 1977a. Morphology, physiology, behaviour and ecology of Perca 
fluviatilis L. and Perca flavescens Mitchill. J. Fish. Res. Board Can. 34 : 1504-1514.

Thorpe J.E., 1977b. Synopsis of biological data on the perch Perca fluviatilis (Linnaeus, 1758) and Perca flavescens (Mitchell, 1814). FAO Fish. Synop. 113 : 138.

Tocko M.N., 1987. The development and nutrition of the young cyprinids of Lake Ohriod. I. Special Edition, Hydrobiological Insitute, Ohrid, pp. 1-165.

Todd A.S., Coleman M.A., Konowal A.M., May M.K., S. Johnson, Vieira N.K.M. \& saunders J.F., 2008. Development of New Water temperature Criteria to Protect Colorado's Fisheries. Am. Fish. Soc. Spec. Publ. 33 (9) : 433-443.

Torgersen C.E., Faux R.N., McIntosh B.A., Poage N.J. \& Norton D.J., 2001. Airborne thermal remote sensing for water temperature assessment in rivers and streams. Rem. Sens. Environ. 76 (3) : 386-398.

Torgersen C.E., Price D.M., Li H.W. \& Mclntosch B.A., 1999. Multiscale thermal refugia and stream habitat associations of chinook salmon in Northeastern Oregon. Ecol. Appl. 9 (1) : 301-319.

Toth E.O., Gulyas P. \& Olah J., 1982. Effect of temperature on growth, food conversion, and survival of sheatfish (Silurus glanis L.) and common carp (Cyprinus carpio L.) at sublethal ammonia concentration. Aquacult. Hung. 3 : 51-56.

Trembley F.J., 1960. Research project on effects of condenser discharge water on aquatic life. The institute of Research, Lehigh University.

Tseitlin V.B., 1980. Duration of gastric digestion in fishes. Mar. Ecol. Progr. 2 : 277-280.

Urho L., 1996. Habitat Shifts of perch larvae as survival strategy. Ann. Zool. Fennici 33 : 329-340.

Vallod D., 1987. Le silure (Silurus glanis, L.). ADAPRA. Lyon, p. 40.

Varley M.E., 1967. British Freshwater Fishes - Factors Affecting their Distribution. London.
Verneaux J., 1981. Les poissons et la qualité des cours d'eau. Annales Scientifiques de l'Université de Franche-Comté (Besançon) 4 (2) : 26-41.

Vladimirov M.Z. \& Bodareu M.N., 1975. Embryological development of the barbel (Barbus barbus). Biol. Resur. vod. Moldav. 13 : 123-139.

Wagner C., Wahl P. \& David H., 2007. Evaluation of temperature-selection differences among juvenile muskellunge originating from different latitudes. Environ. Biol. Fish. 79 (1-2) : 85-98.

Wang N. \& Eckmann R., 1994. Effects of temperature and food density on egg development, larval survival and growth of perch (Perca fluviatilis L). Aquaculture 122 : 323-333.

Wapora I., 1977. Report on the water quality and biological data in the vicinity of the Syl Laskin electric power station, Appendix A. Washington D.C., p. 227.

Watenpaugh D.E., Beitinger T.L. \& Huey D.W., 1985. Temperature tolerance of nitrite-exposed channel catfish. Trans. Amer. Fish. Soc. 114 : 274-278.

Weatherley A.H., 1973. Thermal stress and interrenal tissue in the perh, Perca fluviatilis (Linnaeus) $141: 527-555$.

Webb B.W., 1996. Trends in stream and river temperature. Hydrol. Process. 10 (2) : 205-226.

Wieser W., Forstner H., Schiemer F. \& Mark W., 1988. Growth rates and growth efficiencies in larvae and juveniles of Rutilus rutilus and other cyprinid species : effects of temperature and food in the laboratory and in the field. Can. J. Fisheries Aquat. Sci. 45 (6) : 943-950.

Wiliamson J.H., Carmichael G.J., Graves K.G., Simco B.A. \& Tomasso J.R.J., 1993. Centrarchids. In Culture of nonsalmonid freshwater fishes. R.R. Stickney Ed., pp. 145-198.

Willemsen J., 1959. Research of the production of northern pike, pp. 58-65.

Willemsen J., 1977. The influence of temperature on feeding, growth and mortality of pikeperch and perch. Verh. 
Internat. Verein. Limnol. 20 : 21272133.

Wohlgemuth E., 1981. Nekteré vlastnosti populace horavky duhové (Rhodeus sericeus) z reky Jihlavy. Acta Sci. Nat. Mus. Moraviae Occid. Trebíc 12 : 29-34.

Wolnicki J. \& Gorni W., 1994. Termiczne optimum wzrostu modocianej świnki, Chondrostoma nasus L. Komunikaty Rybackie (2) : 18-19.

Wolnicki J. \& Myszkowski L., 1999. Larval rearing of reophilic cyprinids, Aspius aspius (L.) and Leuciscus cephalus (L.), on live, dry or mixed diet. Europ. Aquacult. Soc., special publication 27 : 258-259.

Wolter C., 2007. Temperature influence on the fish assemblage structure in a large lowland river, the lower Oder River, Germany. Ecol. Freshw. Fish. 16 (4) : 493-503.

Woynarovich E. \& Horvath L., 1981. La reproduction artificielle des poissons en eau chaude: manuel de vulgarisation. FAO, p. 191.

Wurtz-Arlet J., 1950. Quelques observations sur la biologie de la vandoise (Leuciscus leuciscus, L.). Bull. Fr. Piscic. 158 : 14-18.
Wüstemann O. \& Kammerad B., 1995. Der Hasel Leuciscus leuciscus. Die Neue Brehm-Bücherei.

Yildirim A., Erdogan O., Turkmen M. \& Demir B.C., 1999. The investigation of some reproduction characteristics of the Alburnoides bipunctatus fasciatis (Nordman, 1840) living in Oltu Stream, Coruh Basin. Turk. J. Vet. Anim. Sci. 23 : 679-686.

Zahn M., 1963. Jahreszeitliche Veranderungen der Vorzugstemperaturen von Scholle (Pieuronectes platessa Linne) und Bitterling (Rhodeus sericeus Pallas). Verh. Dt. Zool. Ges. : 562-580.

Zarski D., Kucharczyk D., Kwiatkowski M., Targoñska K., Kupren K., Krejszeff S., Jamróz M., Hakuc-Btazowska A., Kujawa R. \& Mamcarz A., 2008. The effect of stocking density on the growth and survival of larval asp, Apsius aspius (L.) and european chub, Leuciscus leuciscus (L.), during rearing under controlled conditions. Arch. Pol. Fish. 16 (4) : 371-381.

Zhdanova N.N., 1966. Lethal temperature limit, and depths required for the artificial rearing of young pike-perch. Tr. Azov. Nauch. Issled. Inst. Rybn. Khoz. 8 : 79-88. 


\section{Sites Internet}

Base de données Fishbase : http://www.fishbase.org/ Université du Minnesota (données sur la grémille) : http://fwcb.cfans.umn.edu/research/ruffe/ginfo/ginfo.htm

Fiches Natura 2000 mises en ligne par le Ministère en charge de l'Écologie :

- Blageon : http://www.ecologie.gouv.fr/IMG/natura2000/habitats/pdf/tome7/1131.pdf

- Toxostome : http://www.ecologie.gouv.fr/IMG/natura2000/habitats/pdf/tome7/1126.pdf

- Bouvière : http://www.ecologie.gouv.fr/IMG/natura2000/habitats/pdf/tome7/1134.pdf

\section{ANNEXE 1}

Tableau des données recueillies sur les tolérances thermiques des 19 espèces de poissons étudiées.

Appendix 1 : table of thermal tolerance data for the 19 fish species studied.

Name : in latin, english and french - noms d'espèce en latin, anglais et fançais

Life stage : stade de vie

Thermal conditions : duration of life stages for embryos and larvae (in degreedays) and associated temperature - durée des stades embryonnaire et larvaire (en degrés jours) et température associée

Thermal tolerance : tolérance thermique (températures minimum, optimum et maximum)

Experimental temperatures : see Figure 1 for the meaning of abreviations températures expérimentales et température d'acclimation (TA) associée (voir paragraphe 2.3.2 et Figure 1)

Lethal $\mathbf{T}^{\circ}$ : températures létales (minimum et maximum)

Oxygen : concentration en dioxygène limite sur $24 \mathrm{~h}$ et température d'acclimation associée (TA)

Location : localisation de l'étude (rivière et pays) Czech Rep. : Czech Republic Fin. : Finland Norw. : Norway Aust. : Australia

$\mathbf{L}$ : tests conducted in laboratory, mesures réalisées en laboratoire

Reference : reference of the data, référence de la donnée

T : type of publication ( $\mathrm{O}$ : observations ou $\mathrm{C}$ : review), type d'article $(\mathrm{O}$ : observations ou $\mathrm{C}$ : compilations)

In : reference of the article where the original reference is cited - référence dans laquelle a été éventuellement trouvée la référence initiale 


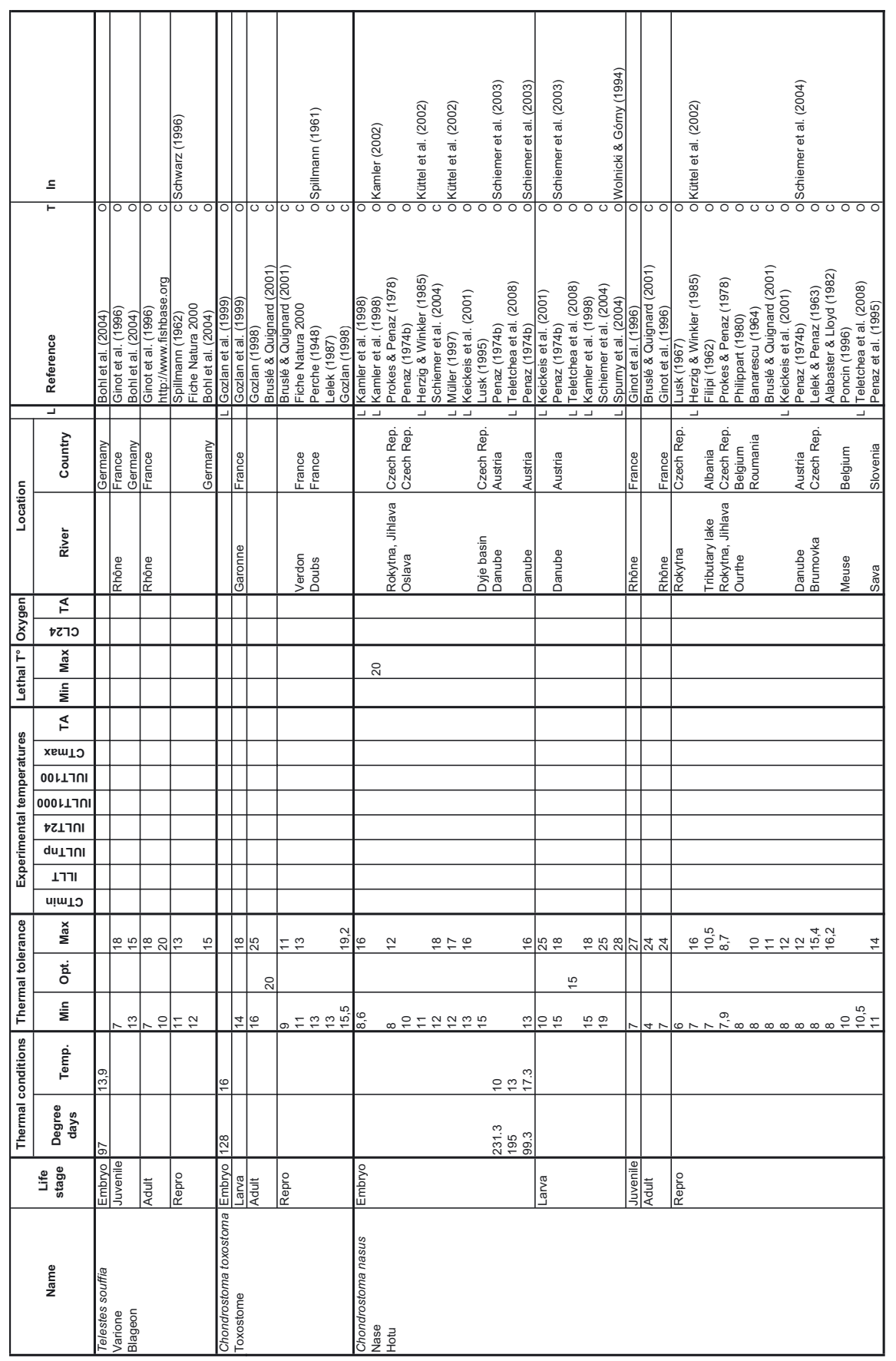




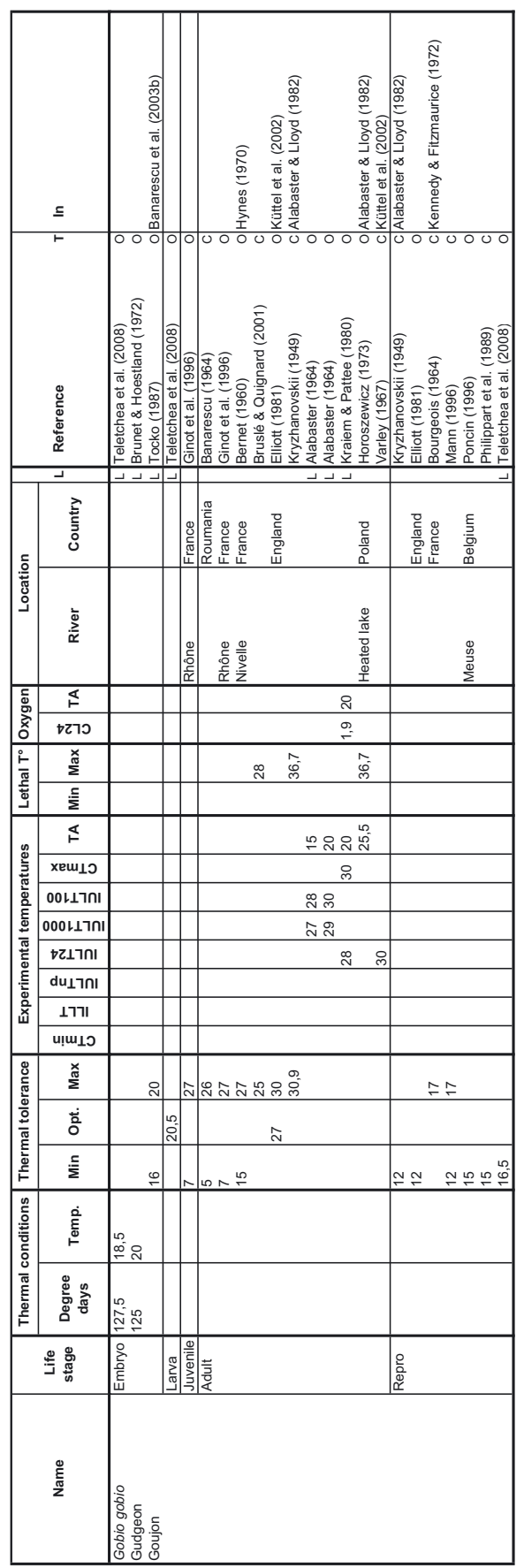




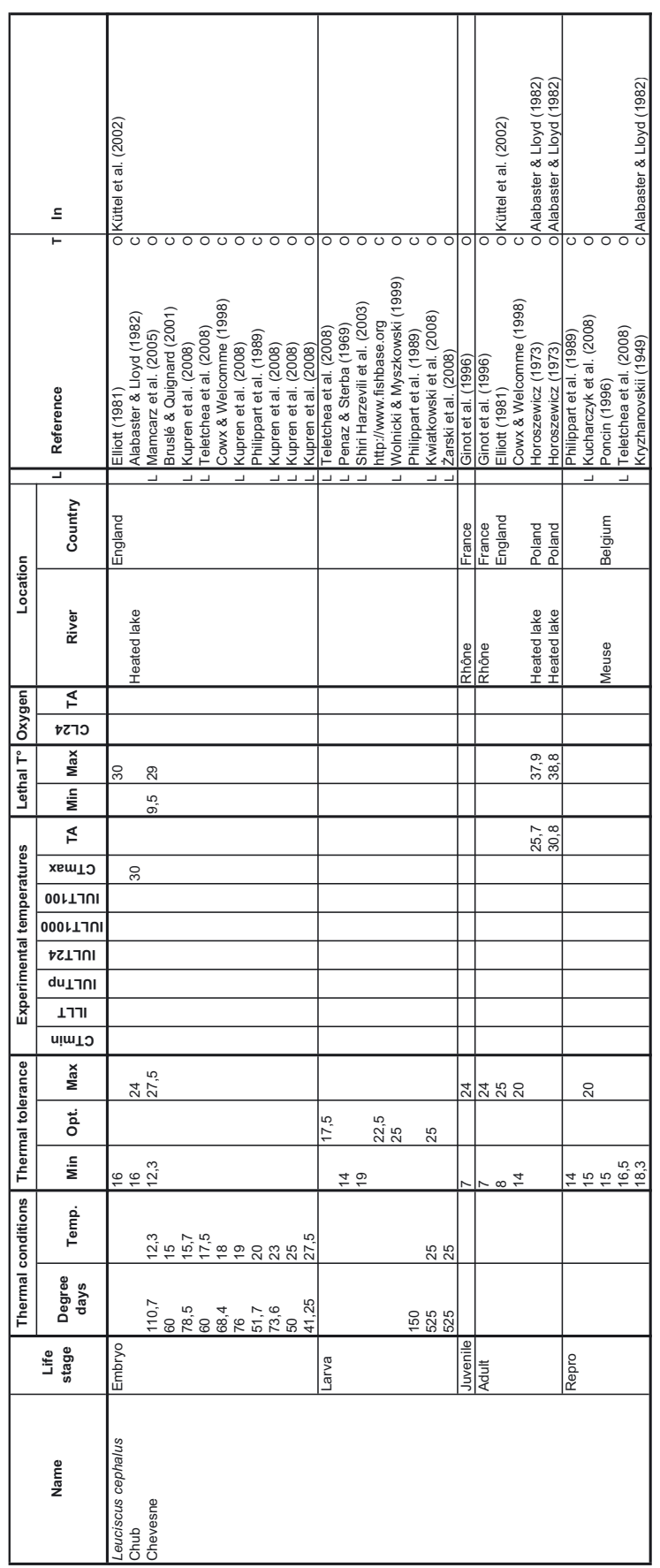




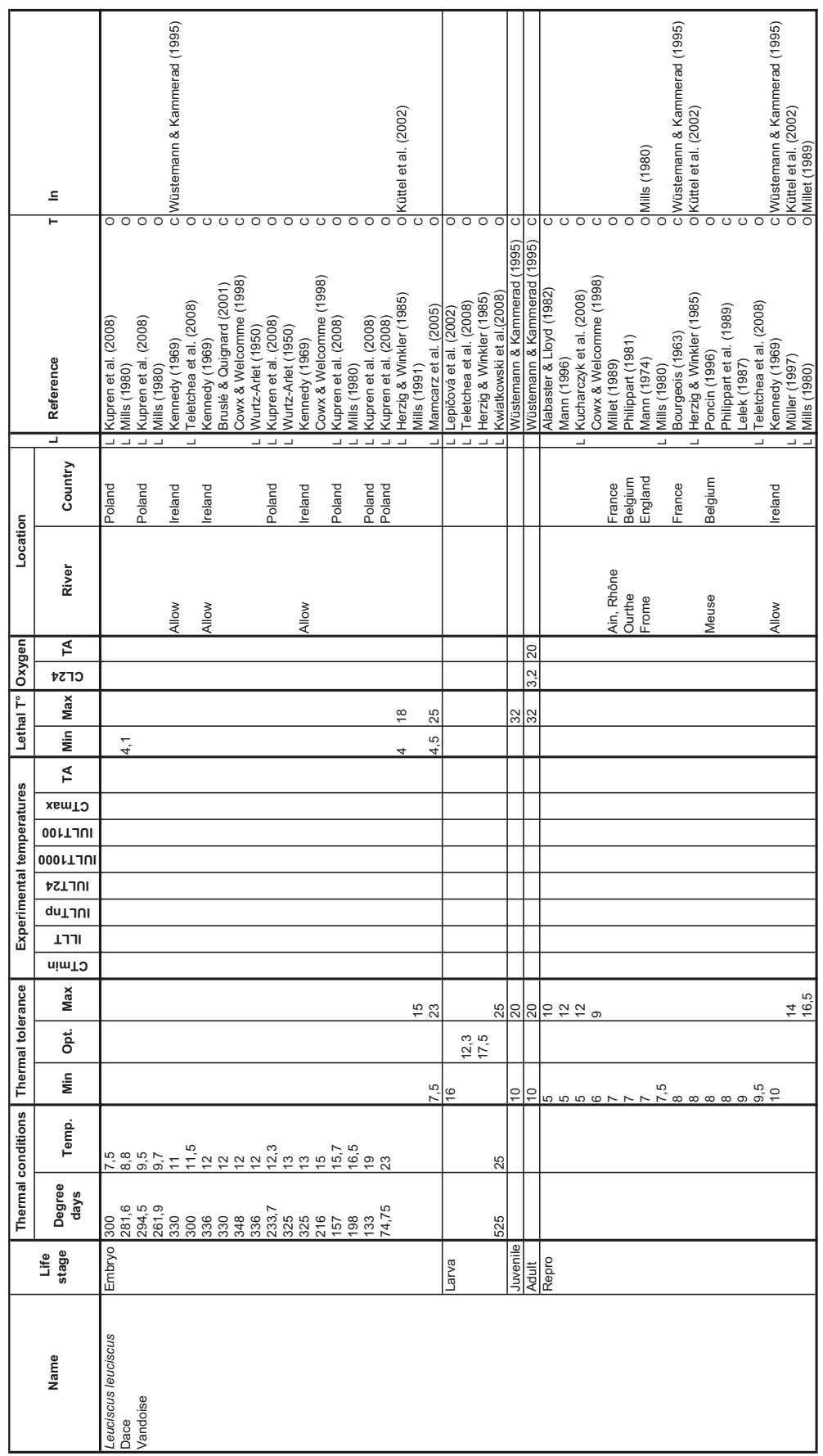




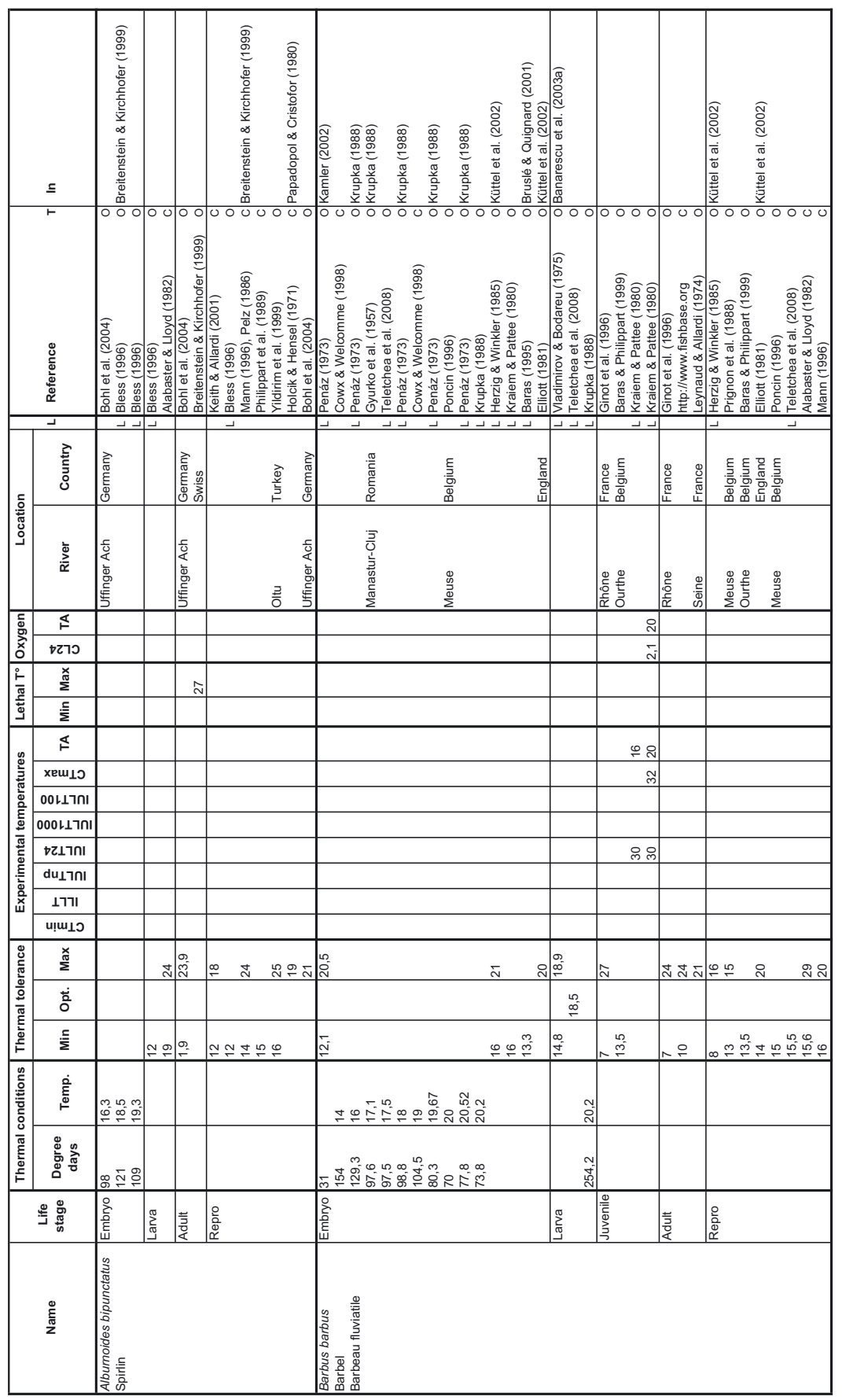




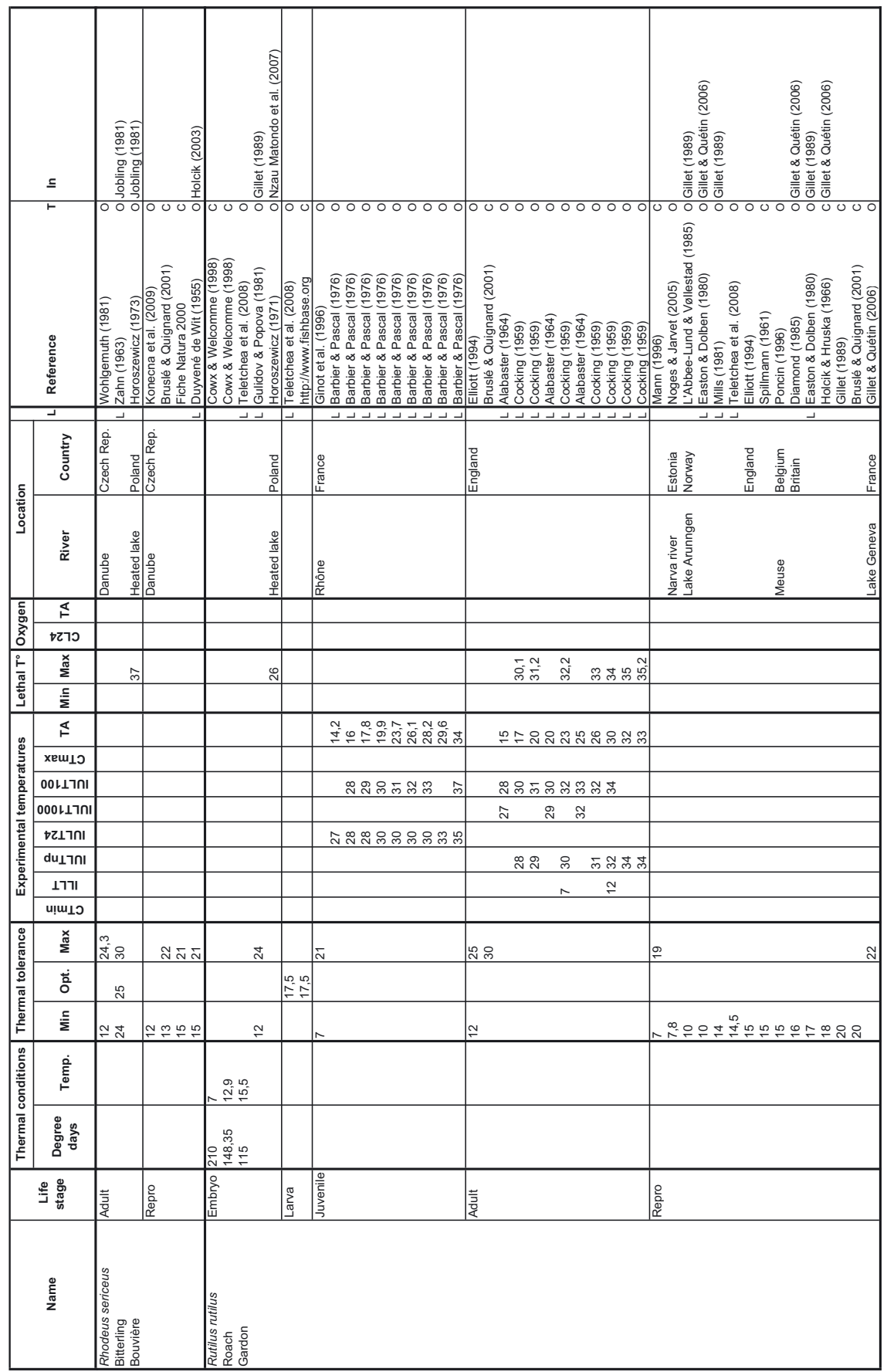




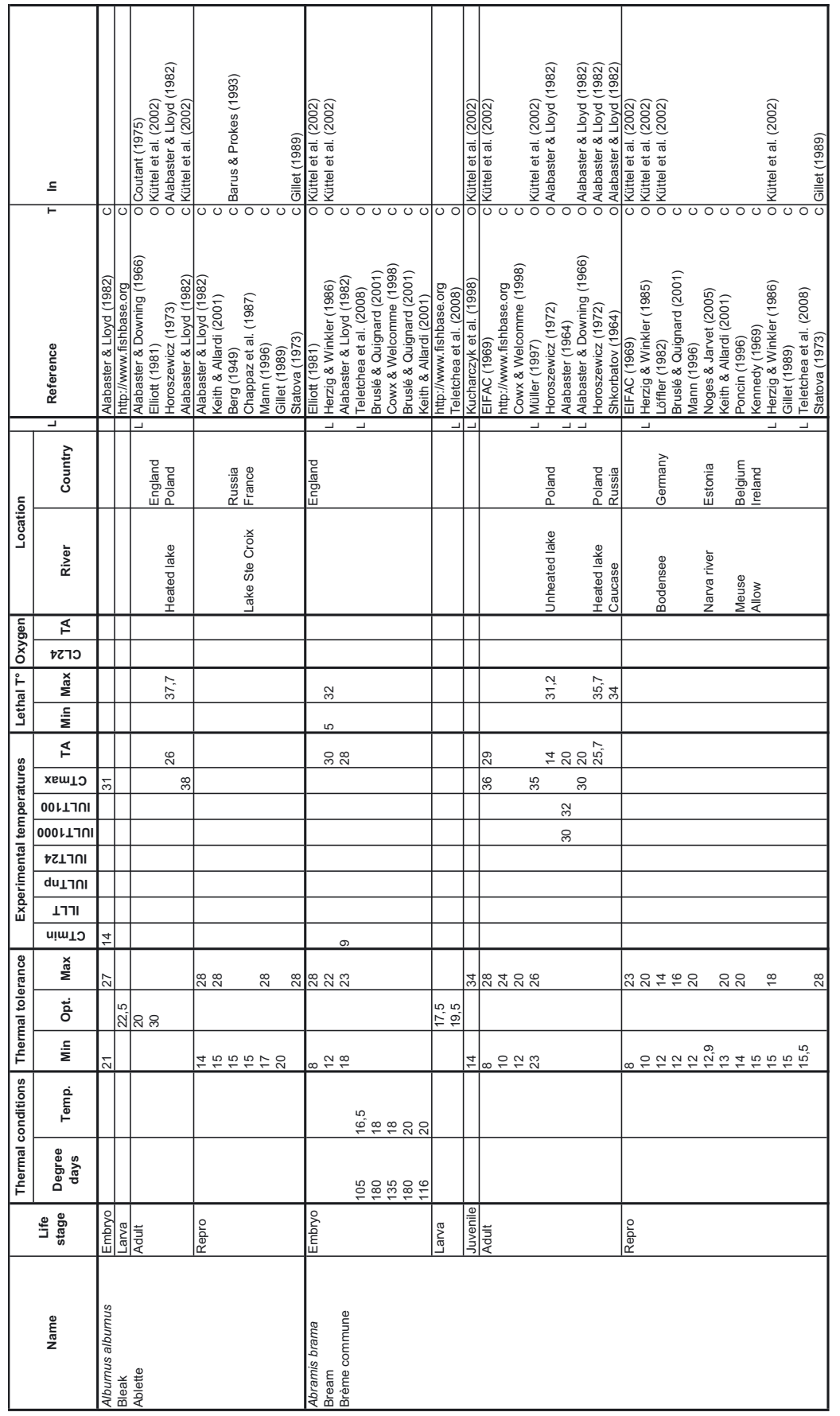




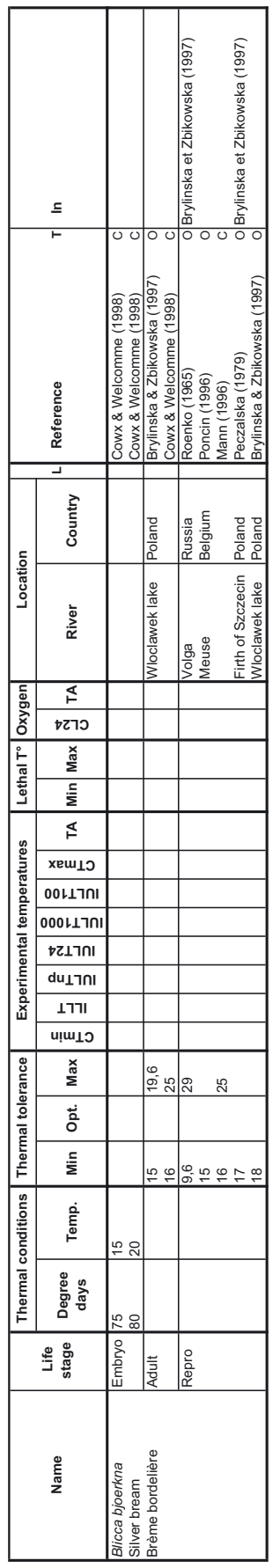




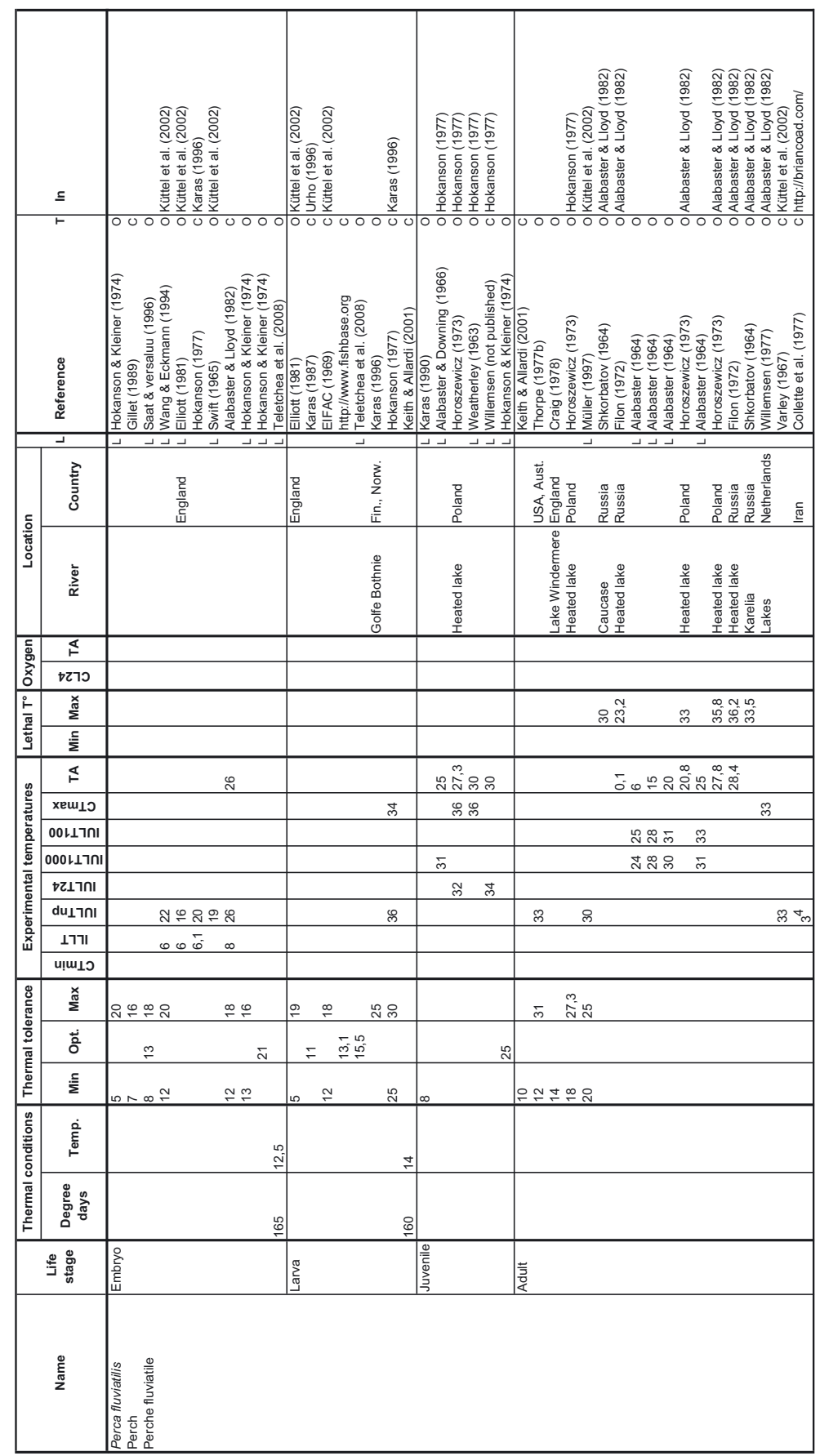




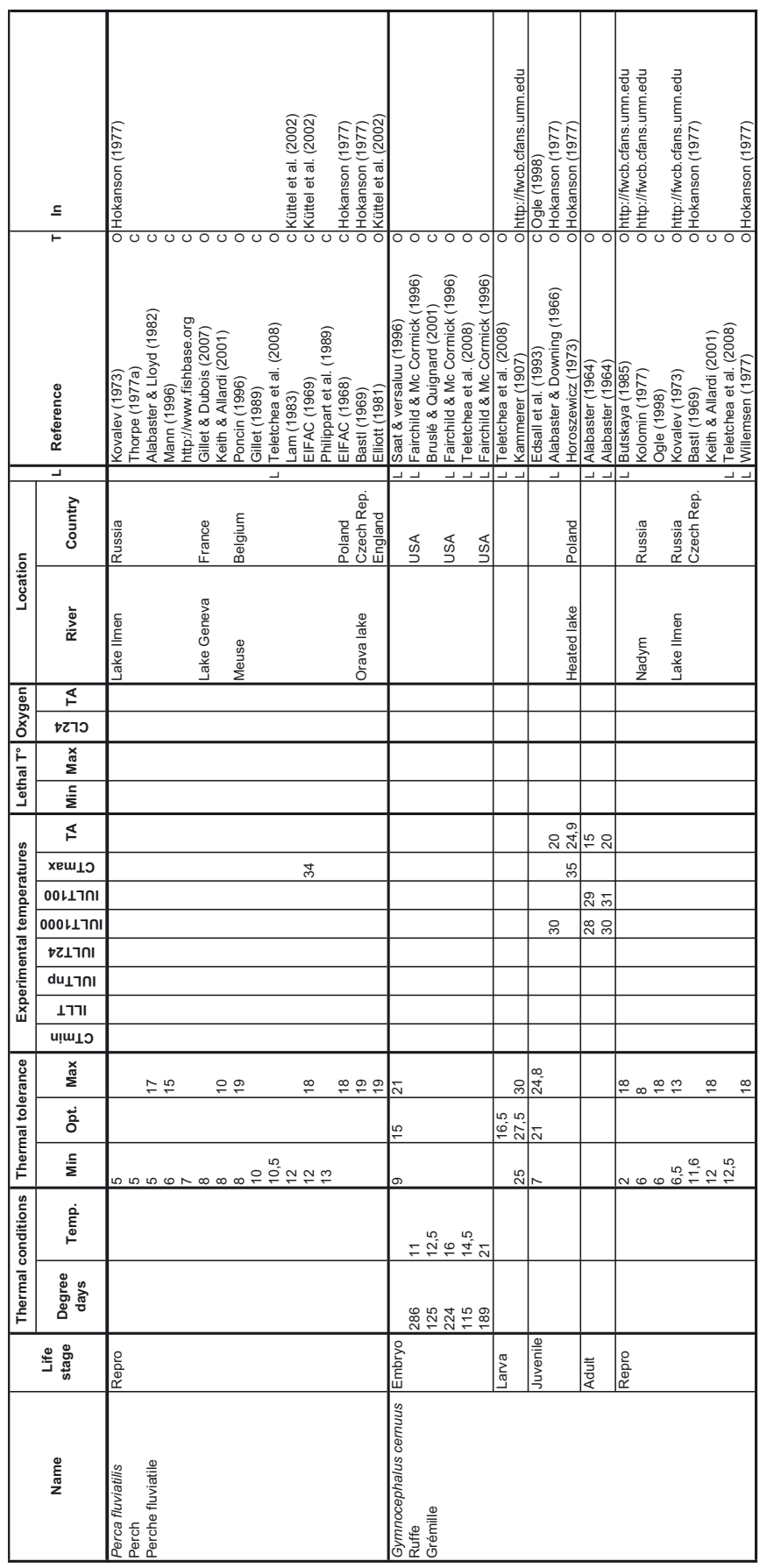




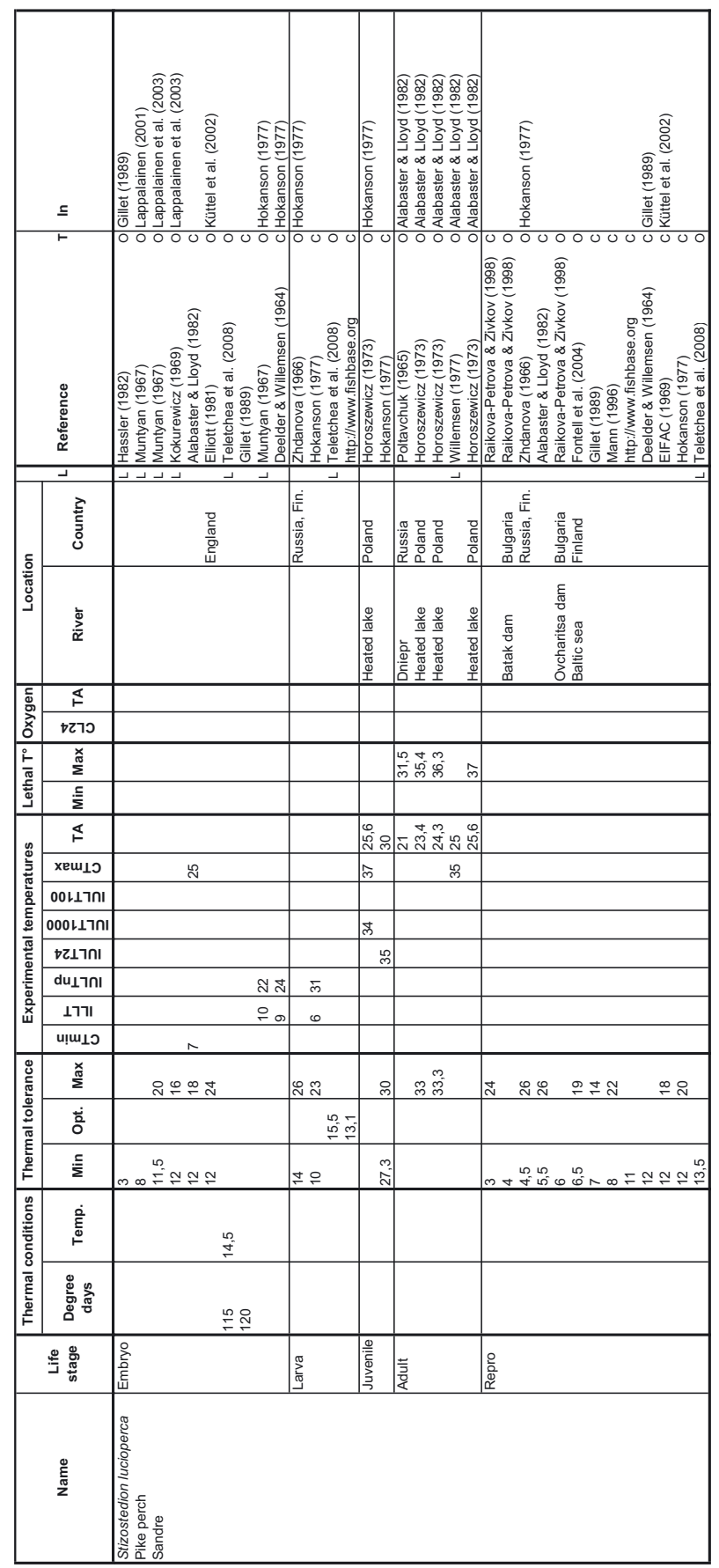




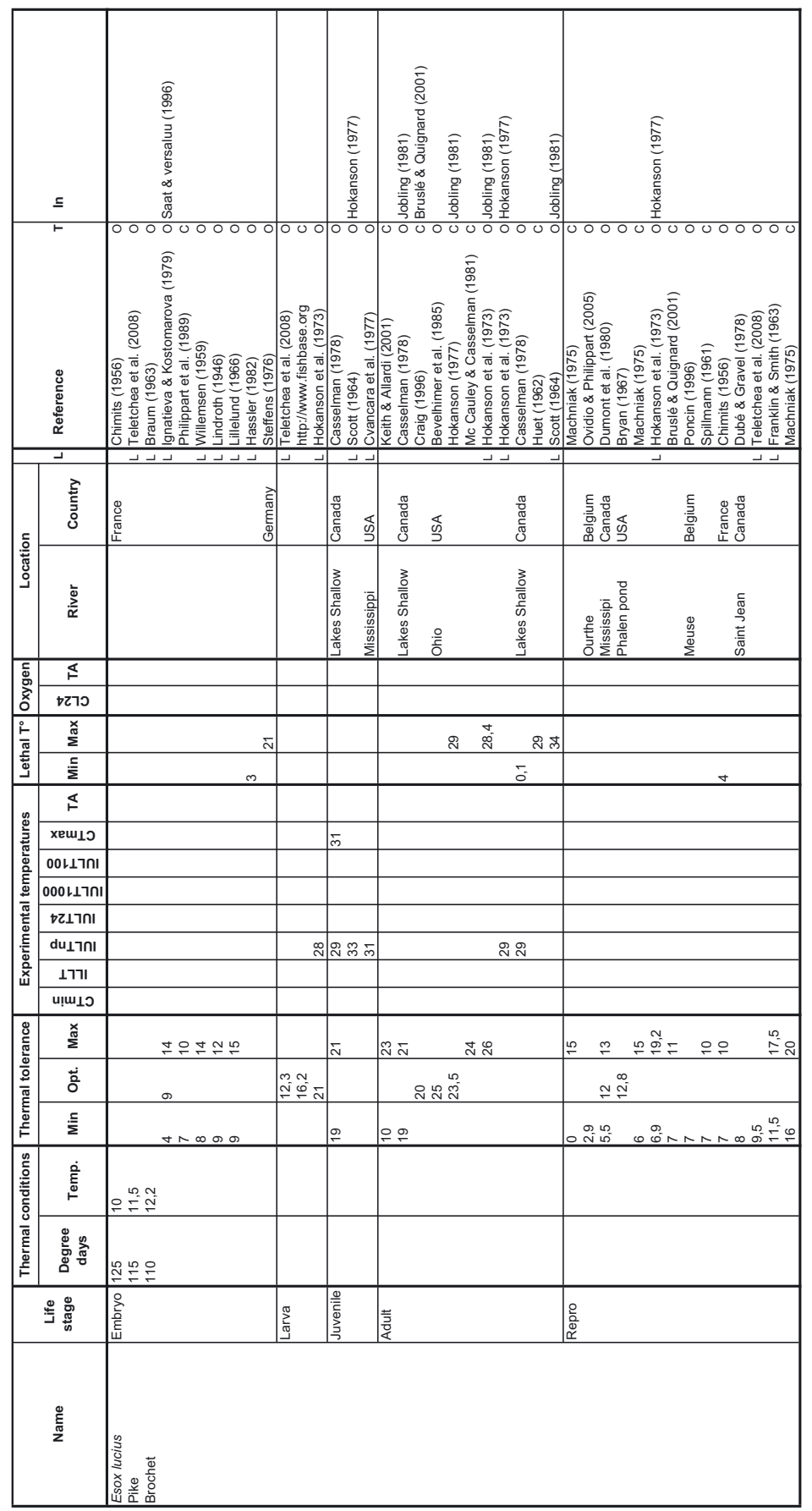




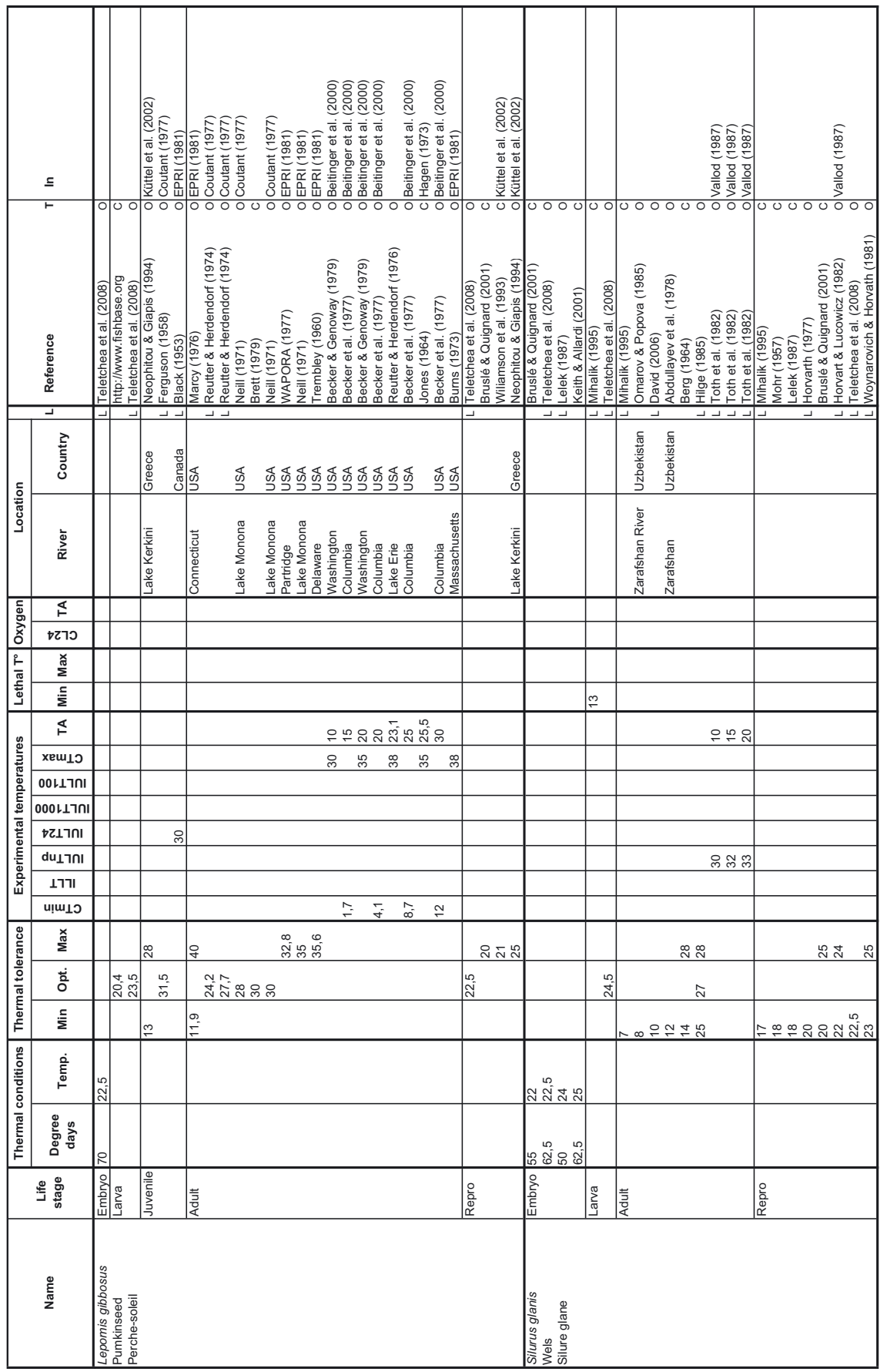

